EPJ Web of Conferences 104, 01002 (2015)

DOI: $10.1051 /$ epjconf/201510401002

(C) Owned by the authors, published by EDP Sciences, 2015

\title{
Diffraction: Principles and application
}

\author{
Thomas C. Hansen \\ Institut Laue-Langevin, 71 avenue des Martyrs, 38000 Grenoble, France
}

\begin{abstract}
We introduce here diffraction in general, as well as neutron and powder diffraction in particular as a tool to study the structure of condensed matter, crystalline solids in particular. Diffraction is a crucial experimental technique of extraordinary potential to elucidate the structure and its evolution of materials important for all domains in the production, conversion, storage and transport of energy. It allows therefore establishing structure-property relationships, which need to be understood in order to develop new and better performing materials.
\end{abstract}

Résumé. Nous introduisons la diffraction en général et celle des neutrons et des échantillons poly-cristallins en particulier en tant qu'outil afin d'étudier la structure de la matière condensée, en particulier des solides cristallisés. Ceci constitue une technique expérimentale de base - et d'un potentiel exceptionnel - pour élucider la structure et son évolution des matériaux importants pour tous les secteurs de la production, de la conversion, du stockage et du transport d'énergie, et permet ainsi d'établir une relation des propriétés et de sa structure dans le but de développer des matériaux encore plus performants.

\section{Diffraction}

The aim of this chapter is the presentation of neutron diffraction as a tool to elucidate the structure of condensed matter, in particular crystalline matter. It is meant as an general introduction for the widespread scientific public from all fields of geosciences, chemistry, materials sciences, metallurgy and mechanical engineering, potentially involved in the characterisation and elaboration of materials for energy production, conversion, transport and storage, potentially interested in application of neutron diffraction to their problems. It is not intended to satisfy all - i.e., mathematical - exigencies of a fullblown condensed matter physicist or a pure crystallographer.

Throughout this chapter one is faced with the eternal problem whether one should start with the egg or with the hen. One could start with some crystallographic notions and introduce then the diffraction tool; one could start with wave functions and derive cross-sections later. There is not a single way, not a single order to understand everything, the diffraction tool in its different declinations and the objects probed by this technique. Therefore I started with an easy mechanistically approach to scattering and diffraction, before diving into a more involved treatment necessary to prepare for the full scope of the method. Then, some crystallographic notions are introduced, which are absolutely necessary to deal with diffraction experiments, before one dives once again in a more complex treatment leading to some

This is an Open Access article distributed under the terms of the Creative Commons Attribution License 4.0, which permits unrestricted use, distribution, and reproduction in any medium, provided the original work is properly cited. 


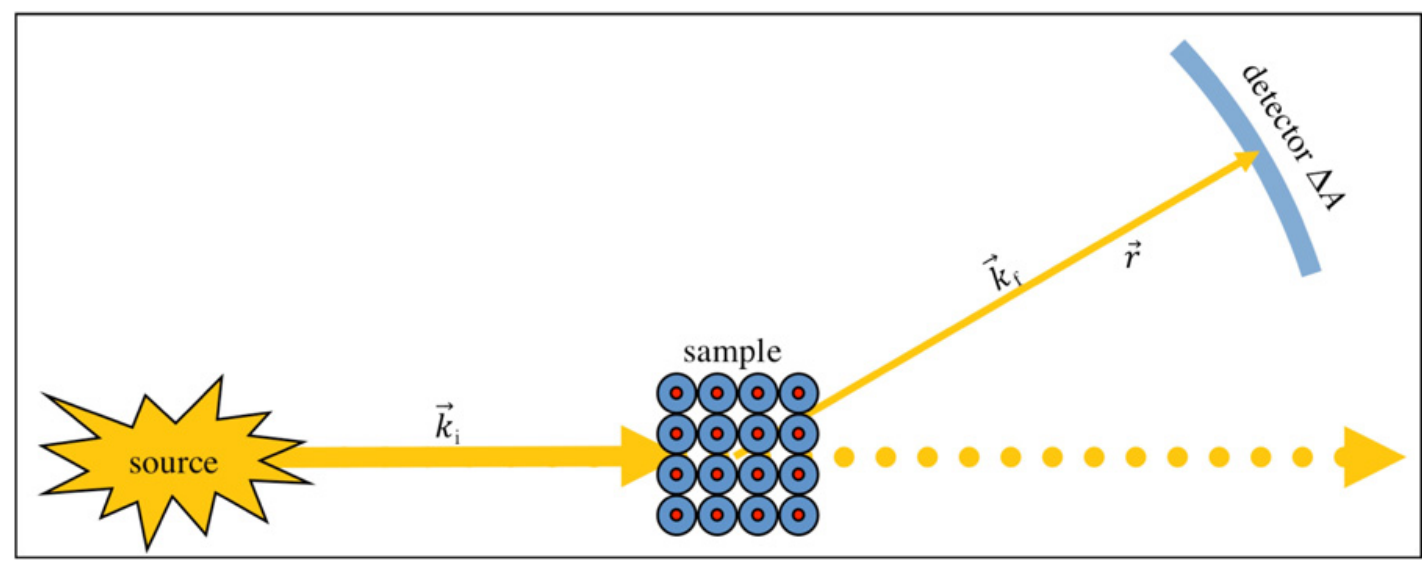

Figure 1. Outline of a scattering experiment: A source emits probing particles arriving at a sample, which scatters some particles in some direction. A detector detects the scattered particles.

specific "neutron issues". Finally, a toolbox is presented making the interpretation of down-to-earth diffraction experiments approachable for scientist in need of this powerful technique. In this approach it was unavoidable to use some terms, e.g., that of "unit cell", to some extend in some context before introducing them properly in another context. An index at the end of this chapter may help the reader to fill gaps if needed.

As diffraction is commonly considered as a special case of scattering, one should start with a general introduction and definition of scattering.

\subsection{Scattering}

One should be aware of the fact that our whole conception of the microscopic world is based on scattering experiments. This includes in particular our knowledge of elementary particles, such as proton and neutron: scattering experiments at high energy elucidate today even their internal structure.

\subsubsection{Definition of scattering}

Scattering is the deflection of radiation (or, rather, propagating waves) ${ }^{1}$ from a straight trajectory by one (bending) or more paths (spreading) when encountering localised non-uniformities, i.e., an object, in the medium through which it passes. All kinds of waves may be scattered, e.g., sound waves, light waves, or particle waves. We may well consider the deflection as caused by collisions (see 1.3, the conception of cross section for this) if we consider the moving particle character of the propagating wave, particularly appropriate in the case of neutrons, and if we consider other particles, such as the atoms of crystalline matter, as the "localised non-uniformities" (or scatterers). The examples are manifold: cosmic rays are scattered by the Earth's atmosphere, electrons by gas atoms in fluorescent lamps and neutrons by the moderator in a nuclear reactor. Scatterers can be elementary particles, all kinds and scales of density fluctuations in condensed matter (bubbles in liquids, droplets in aerosols, pores in solids), defects in crystals, atoms in condensed matter, roughness of surfaces, fibres, etc..

\footnotetext{
${ }^{1}$ Radiation, in its strict physical sense, is the propagation process of energetic waves (or particles) through vacuum or matter without that medium being required for propagation. Waves of the massive medium itself, such as sound waves or ripples on the surface of a liquid are usually not considered as such, although the process of scattering also applies to these waves.
} 
The term "scattering" includes as well a deviation of reflected radiation from the angle predicted by the laws of reflection ${ }^{2}$ and refraction ${ }^{3}$. Reflections undergoing scattering are called diffuse reflections, whereas un-scattered reflections are called specular (mirror like) reflections. The degree of diffuse reflection of light depends on the surface roughness; it is caused by surface irregularities smaller than the wavelength of the incident light.

Scatterers become themselves in turn wave generators when interacting with the incident wave. This is of crucial importance in order to understand any interference (1.2) phenomenon and in particular diffraction (1.3.5).

\subsubsection{Single and multiple scattering}

When radiation is scattered by a single scatterer it is called single scattering. If many scatterers are grouped together, the radiation may scatter many times, which is called multiple scattering. Whilst the earlier can be treated as random phenomenon and described by probability distributions, the latter leads to a deterministic distribution of intensity due to the large number of events averaging out randomness. This has strong analogies to diffusion, which can be interchanged in many contexts with the term "multiple scattering" (and respectively diffusers and multiple scatterers).

\subsubsection{Scattering at atomic scale}

The physics of condensed matter, enclosing solid state physics, is based upon experiments of scattering electromagnetic waves or massive particles (neutrons, positrons, electrons, helium atoms), which help to understand the organisation of matter, thus, its structure, but also the movements of the constituting atoms, its dynamics.

\subsubsection{Microscopy}

The most intuitive technique in order to understand structure is of course the direct observation, which is the domain of microscopy. Electron microscopy goes beyond the limits given by the longer the wavelength of visible light, and approaches - together with X-ray microscopy - the scale of individual atoms by scanning the investigated object. Scanning tunnelling microscopy [1] and atomic force microscopy [2] revolutionised our capacities of observation at atomic scale, but also those of manipulating matter at this level.

Compared to these probing techniques, scattering techniques are less direct, as they convert spatial information about an arrangement in three dimensions to angular information.

\subsubsection{A simple scattering experiment}

Probing particles are sent to a sample. ${ }^{4}$ In the case of thermal ${ }^{5}$ neutron scattering, the sample may change its internal state but stays intact and - in general - no secondary particles are emitted ${ }^{6}$. One will

\footnotetext{
2 The reflected ray lies in the plane of incidence and $\theta_{1}^{\prime}=\theta_{1}$.

3 The refracted ray lies in the plane of incidence and $n_{1} \sin \theta_{1}=n_{2} \sin \theta_{2}$ (Snell's law).

4 The sample does not need to be motionless. In particle collider experiments it may even move at high speed, thus, two beams interact, rather than a beam and a sample.

5 Thermal neutrons have lost their initially high energy after creation in a fission or spallation event through several collisions scattering - in a moderator at roughly ambient temperature. Their kinetic energy corresponds in consequence to ambient temperature, $k T \approx 25 \mathrm{meV}$.

${ }^{6}$ In the rare case of absorption of thermal neutrons, the scattering of a neutron results in an emission of secondary particles, typically high-energy photons (gamma-radiation), electrons or positrons (beta-radiation) or helium nuclei (alpha-radiation).
} 


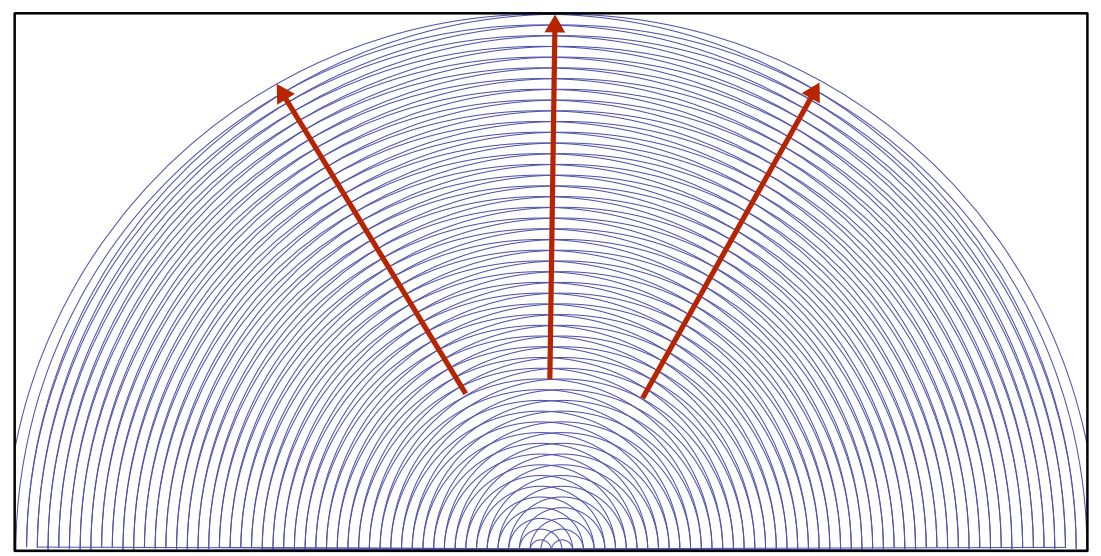

Figure 2. Schematic representation of the interference of two wave sources at a distance of $d=2 \lambda$. One recognizes easily constructive interference at about $2 \theta=\sin ^{-1} \frac{\lambda}{2 d} \approx 29^{\circ}$ left and right of the "incident beam" running from bottom to top with constructive interference. This sketch corresponds to the sketch of the famous double slit experiment Thomas Young presented 1803, the first conclusive proof of the wave nature of light.

now study the diffused probing particles, as all information concerning the sample is contained in the transition probabilities between their initial and its final state. From now on, we will consider this to be the normal scattering case, as it is the one adapted to the investigation of condensed matter.

As the diffusion (multiple scattering) is in our case an interference phenomenon, it is averaging over the illuminated part of the sample. This gauge volume is large as compared to the wavelength of the particles, whereas the requested structural information is of the scale of the wavelength or slightly below. This is the inverse of microscopy, where the gauge volume is small, and the details observed larger than the wavelength.

The probing particles and its states are obviously chosen to fit the needs of the experiment.

The probe must interact sufficiently strongly with the sample in order to be able to observe a noticeable change of state. Thermal neutrons interact with the nuclei of atoms (and with magnetic field). There is no interaction with the electron shell of the atoms; therefore, neutrons are well suited to localise atoms, considering their nuclei as their centres, but not to scrutinise their electronic states directly.

- On the other side, the interaction does not need to be too strong for two reasons:

- The intensity of interaction defines the gauge volume. A strongly interacting particle such as helium atoms, electrons or X-rays has a relatively limited penetration depth: the earlier are scattered at the surface of condensed matter, the latter penetrate further, depending on their energies, without being probes for voluminous, bulk samples. Thermal neutrons are considered ideal probes for bulk materials with the exception of reflectometry experiments, treated in another chapter of this collection.

- The other reason for limited interaction strength is of more mathematical nature: A too strong interaction makes the interpretation of the outcome of a scattering experiment too complex. Multiple scattering ${ }^{7}$ blurs the relationship of sample properties and the final state of the probe. Absence of the later allows the mathematical treatment of the weak interaction after the perturbation theory [3]. The first-order Born approximation (the perturbation method applied to scattering by an extended body, section 3.2) is almost always applicable in neutron scattering,

\footnotetext{
${ }^{7}$ Multiple scattering of one probe on multiple scatterers in a sample, not the interference of multiple probes scattered at the same or at different scatterers in a sample only once.
} 
apart from few cases like the internal total reflection in neutron guides or grazing-incidence small-angle scattering.

- The probing particles need to be scattered in majority instead of being absorbed.

- Absorption hampers the counting statistics of a measurement, due to less scattering events.

- By being absorbed the neutron may deposit its kinetic energy and thus alter the sample, up to the point to destroy it. The method would no longer be a non-destructive one. This problem arises hardly ever for neutrons but is a major concern when it comes to X-ray scattering on soft condensed matter or biological materials, such as proteins.

- The wavelength of the probe determines the spatial dimension that can be probed. As scattering converts assessing of a distance measuring an angle. The wavelength governs the conversion. We shall elucidate this relationship in the following sections on interference and diffraction.

- The total energy of the system of probe and sample shall be conserved. Thus, the change of energy of the probe reflects directly the energy change of the sample. As a matter of fact, the more important the change of a physical value is, the more precisely it is to be quantified, i.e., the change is best at the same scale as the value itself. Probing particles of comparable energy to expected excitations (i.e., phonons $[4,5]$ ) in the sample are thus preferred. The energy scale of excitations in condensed matter depends on its temperature, and the moderated neutrons have energy comparable to a gas at the temperature of the moderator. As a thumb rule, one would examine excitations at a given temperature $T$ with neutrons moderated at the same temperature.

- It is of interest to be able to vary the intensity of the interaction of the probing particle with certain atoms of a condensed matter sample. This provides a possibility to mark the signal coming from certain atoms of the sample. This contrast variation is done, in the case of neutrons, by substituting isotopes by other isotopes of the same element, whereas, in X-ray diffraction, choosing a wavelength close to an absorption edge of a particular element does this.

\subsection{Interference}

When (otherwise identical ${ }^{8}$ ) waves from two $\left(\right.$ or more $^{9}$ ) sources overlap at a point in space, the combined wave intensity at that point can be greater or less than the intensity of either of the waves. This interference can be either constructive when the net intensity is greater than the individual intensities or destructive in the opposite case [4]. When two identical waves arrive at a point in phase, they line up crest-to-crest and valley-to-valley. The resultant wave has twice the amplitude of the component waves, which is the maximal constructive interference. The condition for this is fulfilled whenever the phase difference is $\pm 2 n \pi$ (in radians) or $\pm n \lambda$ with integer $n$ and wavelength $\lambda$. In the other extreme the waves line up crest to valley, which is the condition for complete destructive interference, fulfilled at a phase difference of $\pm(2 n+1) \pi$ or $\pm(n+1 / 2) \lambda$.

Waves, for which the phase relationship between the two waves does not change with time, are called coherent. When they interfere, the intensity of the combined wave at any point in space does not change with time. Coherence is a necessary condition for interference to occur. Two different wave sources ${ }^{10}$ are in general not coherent as the emission of one source is independent of the emission at the other one. These incoherent waves do not maintain a definite phase relationship. In an interference experiment, a wave from a single source must therefore be divided into two components, which can be made to

\footnotetext{
8 The case of sources emitting waves of several wavelengths can be de-convoluted into separate interferences of the individual composing wavelengths.

${ }^{9}$ For more than two sources we shall gradually shift from the term "interference" to "diffraction", see Sect. 1.3.5.

10 The term "radiation" is not used here, as the conception of interference applies also to waves of a massive medium not considered as radiation in a strict sense. ${ }^{1}$
} 


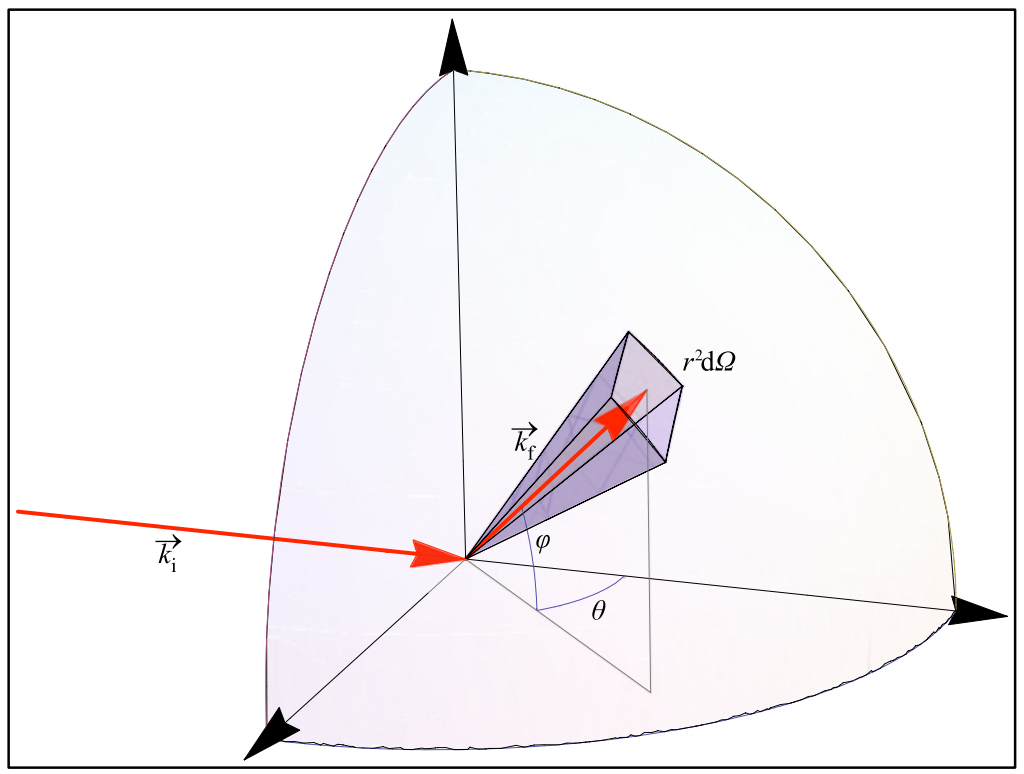

Figure 3. The basic scattering experiment in three dimensions: wave vectors and solid angle $d \Omega$.

interfere, e.g., two identical neighbouring scattering nuclei in a crystal lattice scattering a neutron beam, i.e., becoming two sources of coherent neutron waves. If the two sources emit waves coherently through space, one will have simultaneously constructive interference at some points, the interference maxima, and destructive one at others, the interference minima.

\subsection{Cross section}

A cross section is the effective area that determines the likelihood of scattering or absorption. In nuclear and particle physics this notion is used to express the probability of interaction of particles, as the term is derived fro the classical picture of point-like projectiles shoot at an area including a solid target; the total interaction probability for the single projectile will be the radio of the area of the section of the solid to the targeted area.

\subsubsection{Absorption cross section}

This classical conception can be easily extended to the absorption of neutrons ${ }^{11}$. When a neutron beam travels through matter, a part of the neutrons may be absorbed by the sample's nuclei by exciting them and make them emit a proton, an alpha-particle, a gamma-photon or even make them fission. Provided the sample is a thin plate where the nuclei do not throw shadow on others, the number of such absorption events, $R$, is proportional to the beam intensity $I_{0}$, expressed as neutrons per second $\left(\mathrm{s}^{-1}\right)$ and the concentration of nuclei on the plate, expressed in nuclei per surface unit $\left(\mathrm{cm}^{-2}\right): R=I_{0} n \sigma_{\text {abs }}$. The proportionality constant $\sigma_{\text {abs }}$ has thus the dimension of a surface and is typically expressed in barns ${ }^{12}$,

\footnotetext{
11 By treating absorption we leave the scope (diffraction) of this chapter, but it is useful to look at this phenomenon here, both, to understand the notion of cross sections and, further on, to consider absorption effects properly in neutron diffraction experiments.

12 The name of the unit barn comes from the fact that the scattering cross section of uranium nuclei, examined in the frame of the Manhattan project, has been considered as unusually large: "big as a barn (door)".
} 
where 1 barn $=10^{-24} \mathrm{~cm}^{2}$. Absorption, a phenomenon of all radiations, depends on the wavelength or energy. For neutrons, there are resonance energies at which neutrons create excitations of the composed nucleus with the neutron. Around these energies, the absorption is very strong. For most nuclei, these energies are far above those of thermal neutrons, and the absorption cross section is proportional to the wavelength $\lambda$ and can be extracted and deduced from tables in which one value is given for one wavelength [6], but for those "pathological" nuclei (i.e., ${ }^{3} \mathrm{He},{ }^{6} \mathrm{Li},{ }^{10} \mathrm{~B},{ }^{113} \mathrm{Cd},{ }^{115} \mathrm{In},{ }^{123} \mathrm{Te},{ }^{149} \mathrm{Sm},{ }^{151} \mathrm{Eu}$, ${ }^{155} \mathrm{Gd},{ }^{157} \mathrm{Gd},{ }^{164} \mathrm{Dy},{ }^{168} \mathrm{Yb},{ }^{176} \mathrm{Lu}$ ) having resonances in the energy range of thermal neutrons (as used for diffraction experiments) one needs to consult more exhaustive data $[7,8]$.

\subsubsection{Total cross section}

In the same sense, the scattering cross section is the hypothetical area that describes the likelihood of waves to be scattered by a scatterer, which is, of course, different from the scatterer's geometrical cross section. It depends on the wavelength. The amount of scattering in a sparse medium (no multiple scattering) is proportional to the scattering cross section and the number of scatterers.

The Lambert-Beer law relates the total cross section $\sigma_{\text {tot }}=\sigma_{\text {scat }}+\sigma_{\text {abs }}$ to the absorbance Aof the radiation intensity: $A=c l \sigma_{\text {tot }}$ with the concentration $c$ and the path length $l$. The transmission $T$ depends logarithmically on this: $T=I / I_{0}=\mathrm{e}^{-c l \sigma}=\mathrm{e}^{-\mu l}$, with $\mu=c \sigma$ being the linear absorption coefficient. The later is in the order of 100 to $1000 \mathrm{~cm}^{-1}$ for X-rays (thus, 0.01 to $0.001 \mathrm{~cm}$ of matter are sufficient to attenuate the beam by a factor of 1/e), whereas it is typically between 0.01 and $1 \mathrm{~cm}^{-1}$ for (thermal) neutrons (respectively $1 \mathrm{~cm}$ to $1 \mathrm{~m}$ penetration).

\subsubsection{Scattering cross section}

The only observable in a scattering experiment is the intensity of probe particles as measured by detectors. Let each detector be oriented with its detection area $\Delta A$ perpendicular to the vector $\vec{r}=(r, \theta, \varphi)$. Let the detector area be a part of a sphere or radius $r$ (see Fig. 3). The number of particles $I$ intercepted by a detector of $100 \%$ efficiency at a given time $t$ will be done by the following expression.

$$
I(t)=\Delta A \cdot J_{\text {scatter }}(r, \theta, \varphi, t)
$$

$J$ is the flow of scattered particles, which is radial at sufficient distance from the sample. $J$ is the number of probing particles travelling through a surface unit per time unit. At sufficient distance from the sample, the probe particles can be regarded a free, characterised by its momentum $\hbar \vec{k}$. The wave vector $\vec{k}_{\mathrm{f}}$ of detected scattered neutrons runs parallel to $\vec{r}$ and the incident beam is characterised by its wave vector $\vec{k}_{\mathrm{i}}{ }^{13}$. Scattering corresponds thus to the transfer of a particle initially in a free state $\vec{k}_{\mathrm{I}}$ into a final state $\vec{k}_{\mathrm{f}}$. The flow diminishes as one retreats from the sample, the intensity counted in a detector of a given surface diminishes with $1 / r^{2}$. The dependency of the flow scattered as a function of a direction contains valuable information, thus, the detectable flow in an angular element $d \Omega=\sin \theta \mathrm{d} \theta \mathrm{d} \varphi$. The connection between the detector's surface $-A(\theta, \varphi)$ - and the solid angle $\Omega$ is given by the following relationship.

$$
\Delta A=r^{2} \Delta \Omega
$$

One consequence of this is that a detector of a given surface $\Delta A$ should be placed far enough from the sample to resolve the scattering direction with sufficient precision. What is of interest is the scattered

\footnotetext{
13 In a real experiment, as we will see in the section on instrumentation, one will not work with a single incident wavelength, but with a well-chosen distribution of wave-vectors around a nominal value.
} 
flow in an element of solid angle $d \Omega$ compared to the initial flow at the sample.

$$
d \sigma=\frac{I(t)}{J_{i}(t)}=r^{2} d \Omega \frac{J_{\text {scatter }}(r, \Theta, \phi, t)}{J_{i}(t)}
$$

The quantity $d \sigma / d \Omega$ is called differential (scattering) cross section. It has the dimension of a surface. The integral over all angles gives the scattered flow compared to the initial flow, nothing else but the (total) scattering cross section.

$$
\sigma_{t}=\int \frac{d \sigma}{d \Omega} d \Omega=\int d \sigma
$$

As for the absorption cross section, the scattering cross section is the surface the scatterer opposes the radiation, and it is expressed in barns $\left(1 \mathrm{barn}=10^{-24} \mathrm{~cm}^{2}\right)$. A non-transparent sphere of radius $r$ has a cross section of $\pi r^{2}$ in a homogeneous flow of particles.

\subsubsection{Partial differential scattering cross section}

What has been neglected until now is the possibility in our basic scattering experiment, to filter the scattered probing particles before detection. One filtering of widespread use is that after the probing particles' energies (as the particles can exchange energy with the sample, and thus lower or increase their energy). Such a filter (or analyser) allows for the identification of particles having energies between $E_{f}$ and $E_{f}+d E_{f}$. This leads to the partial ordouble differential scattering cross section, where $\Psi_{\text {scatter }}\left(r, \Theta, \phi, t, E_{f}\right) d E_{f}$ is the flow scattered in an element $d \Omega$ of solid angle with energies between $E_{f}$ and $d E_{f}$.

$$
\frac{d^{2} \sigma}{d \Omega d E_{f}}=\frac{\Psi_{\text {scatter }}\left(r, \Theta, \phi, t, E_{f}\right) d E_{f}}{\Psi_{i}(t)}
$$

The double cross section has the dimension of surface over energy. Double integration over solid angle $\Omega$ and energy $E_{f}$ it delivers the total cross section $\sigma_{t}$.

$$
\sigma_{t}=\iint \frac{d^{2} \sigma}{d \Omega d E_{f}} d \Omega d E_{f}
$$

\subsubsection{Scattering and absorption cross sections for neutrons}

Despite the intention to describe the phenomenon of diffraction and its surrounding as neutral as possible, at least at this early stage, by considering all kinds of scatterers and of probing waves, one cannot hide totally, that the chapter is set in the context of a school about neutron scattering, and therefore finally dedicated to the diffraction of neutrons by condensed matter, consisting of atoms acting as scatterers. Therefore it is appropriate here to show here a selection of scattering and absorption crosssections in order to get an idea for easy and difficult diffraction experiments (Fig. 4 and Table 1). It has already been mentioned in Sect. 1.1.5: thermal neutrons are scattered at the nucleus of atoms, totally independent of the electron shell, but dependent on the isotope, not just the element. However, one can specify an element's cross-section by considering the natural abundance of isotopes. Also, there is no proportionality to the atom mass, or rather the number of electrons per atom as in the X-ray case. The typical scattering cross sections are a few barns $\left(10^{-24} \mathrm{~cm}\right)$, which immediately evokes the "diameter" of atomic nuclei ranging from 2 to $15 \mathrm{fm}$. The table comes somehow too early, as one would need the 


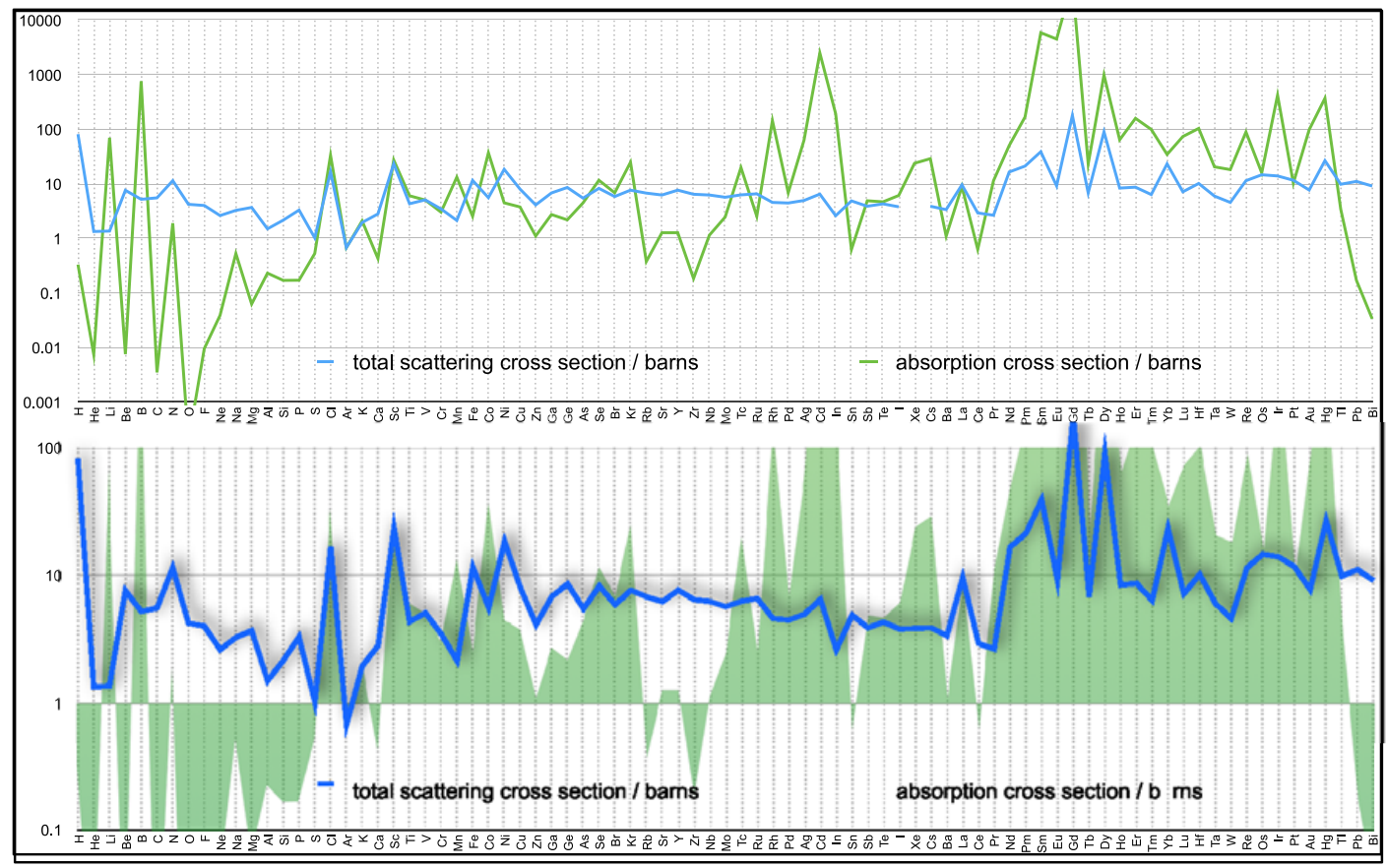

Figure 4. Total scattering (blue) and absorption (green) cross-sections for the stable elements of natural abundance. One notes, e.g., the lowest absorption cross section for oxygen, followed by carbon, beryllium and helium, very high ones for boron, cadmium and gadolinium, consequently used as neutron absorbers. The absorption of other rare earths such as samarium and europium is remarkable, as well as the one of other elements, such as lithium, indium, rhodium, iridium, gold and mercury, whereas the heaviest stable elements, i.e., lead and bismuth, have a low absorption cross section. In the equally logarithmic plot at the bottom, the scale emphasises the scattering cross sections, particularly high for hydrogen, nitrogen, chorine, scandium, iron and nickel, as well as some of the highly absorbing heavier elements. Argon has the lowest scattering cross section, followed by sulphur, helium and lithium. Although an element like lithium has a high absorption and low scattering cross section, neutron diffraction experiments are still possible, but isotopic substitution by the non-absorbing isotope, i.e., ${ }^{7} \mathrm{Li}$, becomes advisable.

Table 1. Neutron total scattering and absorption cross-sections (in barns) for selected elements and isotopes.

\begin{tabular}{|l|r|l|r|r|r|}
\hline Element & A & Z & $\%$ & $\sigma$ & 吕sorption \\
\hline $\mathrm{H}$ & & 1 & & 82.0 & 0.3 \\
\hline $\mathrm{H}$ & 1 & 1 & 100.0 & 82.0 & 0.3 \\
\hline $\mathrm{H}$ & 2 & 1 & 0.0 & 7.6 & 0.0 \\
\hline $\mathrm{He}$ & & 2 & & 1.3 & 0.0 \\
\hline $\mathrm{He}$ & 3 & 2 & 0.0 & 6.0 & 5333.0 \\
\hline $\mathrm{He}$ & 4 & 2 & 100.0 & 1.3 & 0.0 \\
\hline $\mathrm{Li}$ & & 3 & & 1.4 & 70.5 \\
\hline $\mathrm{Li}$ & 6 & 3 & 7.5 & 1.0 & 940.0 \\
\hline $\mathrm{Li}$ & 7 & 3 & 92.5 & 1.4 & 0.0 \\
\hline $\mathrm{Be}$ & & 4 & 100.0 & 7.6 & 0.0 \\
\hline $\mathrm{B}$ & & 5 & & 5.2 & 767.0 \\
\hline $\mathrm{B}$ & 10 & 5 & 20.0 & 3.1 & 3835.0 \\
\hline $\mathrm{B}$ & 11 & 5 & 80.0 & 5.8 & 0.0 \\
\hline $\mathrm{C}$ & & 6 & & 5.6 & 0.0 \\
\hline $\mathrm{N}$ & & 7 & & 11.5 & 1.9 \\
\hline $\mathrm{O}$ & & 8 & & 4.2 & 0.0 \\
\hline $\mathrm{F}$ & & 9 & 100.0 & 4.0 & 0.0 \\
\hline $\mathrm{Na}$ & & 11 & 100 & 3.3 & 0.5 \\
\hline $\mathrm{Mg}$ & & 12 & & 3.7 & 0.1 \\
\hline $\mathrm{Al}$ & & 13 & 100.0 & 1.5 & 0.2 \\
\hline $\mathrm{Si}$ & & 14 & & 2.2 & 0.2 \\
\hline $\mathrm{P}$ & & 15 & 100.0 & 3.3 & 0.2 \\
\hline $\mathrm{S}$ & & 16 & & 1.0 & 0.5 \\
\hline $\mathrm{Cl}$ & & 17 & & 16.8 & 33.5 \\
\hline $\mathrm{Ar}$ & & 18 & & 0.7 & 0.7 \\
\hline & & & & \\
\hline
\end{tabular}

\begin{tabular}{|l|l|l|l|r|r|}
\hline Element & A & Z & $\%$ & $\sigma$ & $\sigma_{\text {absorption }}$ \\
\hline $\mathrm{K}$ & & 19 & & 2.0 & 2.1 \\
\hline $\mathrm{Ca}$ & & 20 & & 2.8 & 0.4 \\
\hline $\mathrm{Sc}$ & & 21 & 100.000 & 23.5 & 27.5 \\
\hline $\mathrm{Ti}$ & & 22 & & 4.4 & 6.1 \\
\hline $\mathrm{V}$ & & 23 & & 5.1 & 5.1 \\
\hline $\mathrm{V}$ & 50 & 23 & 0 & 7.8 & 60.0 \\
\hline $\mathrm{Cr}$ & & 24 & & 3.5 & 3.1 \\
\hline $\mathrm{Mn}$ & & 25 & 100.000 & 2.2 & 13.3 \\
\hline $\mathrm{Fe}$ & & 26 & & 11.6 & 2.6 \\
\hline $\mathrm{Co}$ & & 27 & 100.0 & 5.6 & 37.2 \\
\hline $\mathrm{Ni}$ & & 28 & & 18.5 & 4.5 \\
\hline $\mathrm{Ni}$ & 58 & 28 & 68.3 & 26.1 & 4.6 \\
\hline $\mathrm{Ni}$ & 60 & 28 & 26.1 & 1.0 & 2.9 \\
\hline $\mathrm{Cu}$ & & 29 & & 8.0 & 3.8 \\
\hline $\mathrm{Zr}$ & & 40 & & 6.5 & 0.2 \\
\hline $\mathrm{Nb}$ & & 41 & 100.0 & 6.3 & 1.2 \\
\hline $\mathrm{Rh}$ & & 45 & 100 & 4.6 & 144.8 \\
\hline $\mathrm{Ag}$ & & 47 & & 5.0 & 63.3 \\
\hline $\mathrm{Cd}$ & & 48 & & 6.5 & 2520.0 \\
\hline $\mathrm{Cd}$ & 112 & 48 & 24.1 & 5.1 & 2.2 \\
\hline $\mathrm{Cd}$ & 113 & 48 & 12.2 & 12.4 & 20600.0 \\
\hline $\mathrm{Cd}$ & 114 & 48 & 28.7 & 7.1 & 0.3 \\
\hline $\mathrm{In}$ & & 49 & & 2.6 & 193.8 \\
\hline $\mathrm{In}$ & 113 & 49 & 4.3 & 3.7 & 12.0 \\
\hline $\mathrm{In}$ & 115 & 49 & 95.7 & 2.6 & 202.0 \\
\hline & & & & \\
\hline
\end{tabular}

\begin{tabular}{|c|c|c|c|c|c|}
\hline Element & A & Z & $\%$ & $\sigma$ & $\sigma_{\text {absorption }}$ \\
\hline 1 & & 53 & 100.0 & 3.8 & 6.2 \\
\hline Xe & & 54 & & --- & 23.9 \\
\hline $\mathrm{Xe}$ & 124 & 54 & 0.1 & -- & 165.0 \\
\hline $\mathrm{Sm}$ & & 62 & & 39.0 & 5922.0 \\
\hline Sm & 149 & 62 & 13.9 & 200.0 & 42080.0 \\
\hline Sm & 154 & 62 & 22.6 & 11.0 & 8.4 \\
\hline $\mathrm{Eu}$ & & 63 & & 9.2 & 4530.0 \\
\hline $\mathrm{Eu}$ & 151 & 63 & 47.8 & 8.6 & 9100.0 \\
\hline $\mathrm{Eu}$ & 153 & 63 & 52.2 & 9.8 & 312.0 \\
\hline Gd & & 64 & & 180.0 & 49700.0 \\
\hline Gd & 157 & 64 & 15.7 & 1044.0 & 259000.0 \\
\hline Gd & 158 & 64 & 24.8 & 10.0 & 2.2 \\
\hline $\mathrm{Hf}$ & & 72 & & 10.2 & 104.1 \\
\hline Ir & & 77 & & 14.0 & 425.0 \\
\hline $\mathrm{Au}$ & & 79 & 100.0 & 7.8 & 98.7 \\
\hline $\mathrm{Hg}$ & & 80 & & 26.8 & 372.3 \\
\hline $\mathrm{Hg}$ & 199 & 80 & 17.0 & 66.0 & 2150.0 \\
\hline $\mathrm{Hg}$ & 202 & 80 & 29.6 & 9.8 & 4.9 \\
\hline $\mathrm{Pb}$ & & 82 & & 11.1 & 0.2 \\
\hline $\mathrm{Bi}$ & & 83 & 100.0 & 9.2 & 0.0 \\
\hline$U$ & & 92 & & 8.9 & 7.6 \\
\hline U & 235 & 92 & 0.7 & 14.0 & 680.9 \\
\hline U & 238 & 92 & 99.3 & 8.9 & 2.7 \\
\hline $\mathrm{Pu}$ & 239 & 94 & $24100 a$ & 7.7 & 1017.3 \\
\hline
\end{tabular}


notion of scattering length (section 0) and the nuclear spin and isotope dependence thereof to present important coherent and incoherent scattering cross sections as well, but this shall be treated later in Sects. 3.7 and 3.8. The total scattering length alone does not allow a full appreciation of the expected outcome of a diffraction experiment for which, roughly, only the coherent scattering transports information while any incoherent scattering is mostly considered as a nuisance.

One needs to mention here that cross sections depend on the probe's wavelength. This energy dependence can be estimated with the simple Ramsauer model, where the probe's wavelength $\lambda$ comes in as its effective radius, and thus, the cross section $\sigma$ becomes proportional to the square of the sum of the effective scatterer radius $R$ and the wavelength: $\sigma \propto(R+\lambda)^{2}$. For diffraction with thermal neutrons, $R$ becomes negligible small as compared to the wavelength, so that $\sigma \propto \lambda^{2}$. Also, one may take into account a proportionality with temperature: $\sigma \propto 1 / \sqrt{ } T$. Neutron cross sections are typically given for thermal neutrons of given wavelength and room temperature.

\subsection{Crystal diffraction}

It is difficult to give a neat definition of diffraction, versus interference and scattering, and one finds relief with famous Richard Feynman, who stated that no one has ever defined the difference between interference and diffraction satisfactorily, and that it is just a question of usage, as there is no specific, important physical difference between them: One typically calls the result of only a few sources interfering "interference", and more frequently "diffraction" when there is a large number of sources [9]. Diffraction occurs for all kinds of waves: sound waves, light waves, or particle waves, such as neutrons. It can be a result of coherent scattering: for diffraction to occur, the size of the object or rather the distance of scatterers must be of the order of wavelength of the incident waves. However, it does not need to be scattering leading to diffraction: an ensemble of sources (as evoked by Feynman), emitting waves coherently, results in a similar interference phenomenon.

\subsubsection{Bragg diffraction}

What is of interest for investigating the structure of condensed matter is obviously the diffraction on a crystal: a three-dimensional ${ }^{14}$ periodic structure. The simplest approach to this is surely the law, which W. H. Bragg and his only 22 years old son ${ }^{15}$ derived in 1912 . We consider scattering at the virtual planes formed by the atoms of an infinite periodic lattice as specular (mirror like) reflection with incidence angle equal reflection angle. We assume elastic scattering, i.e., no exchange of energy, incident and reflected waves having the same wavelength. For constructive interference the path difference between reflected rays must be equal to an integral number $n$ of wavelengths $\lambda$ (Fig. 5).

$$
n \lambda=2 d \sin \theta
$$

An alternative derivation is shown in Fig. 6. Bragg diffraction peaks occur only for $\lambda<2 d$, on the other side $\lambda$ and $d$ need to be comparable for peaks to be resolved. Also, with an ideal perfect crystal, a hypothetically infinite periodic structure, one obtains totally destructive interference for any direction that does not fulfil Bragg's law. In reality, the transition from constructive to totally destructive interference is gradual with angle, as crystals have a limited size (beside other deviations from the idealised model, i.e., defects and atomic motion, and incoherent scattering).

\footnotetext{
${ }^{14}$ For Bragg-like diffraction to happen is not necessary to have a three-dimensional structure. The conception stands also for less dimensions, i.e., one can conceive diffraction experiments on the two-dimensional periodic structure of monoatomic layers of absorbed molecules on a substrate. Also, one can describe diffraction on quasi-crystals as diffraction on a periodic structure of more than three dimensions.

15 Father and son received the Nobel Prize for this discovery in 1915, making them the only father and son team ever having received this distinction, and W. L. Bragg with 25 years the youngest laureate ever.
} 


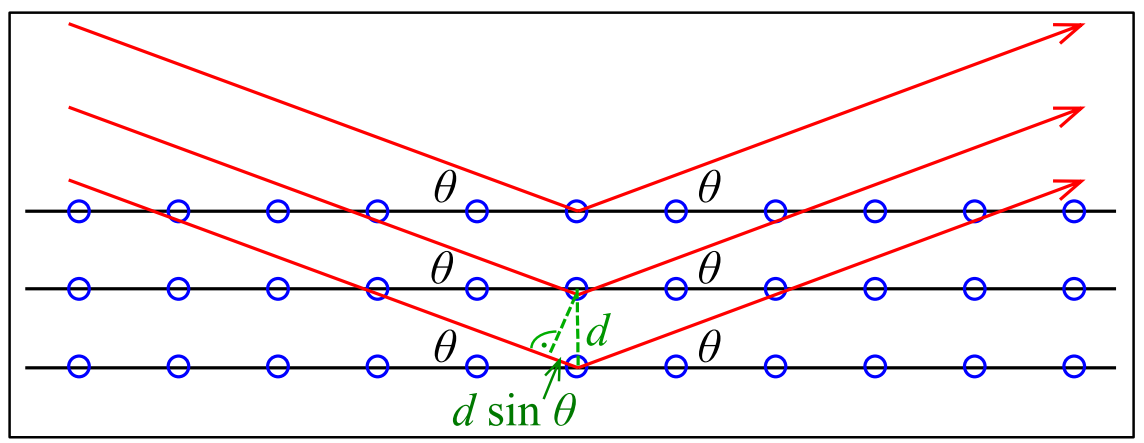

Figure 5. Bragg diffraction as specular reflection on semi-transparent mirrors of lattice planes: constructive interference only for certain path differences.

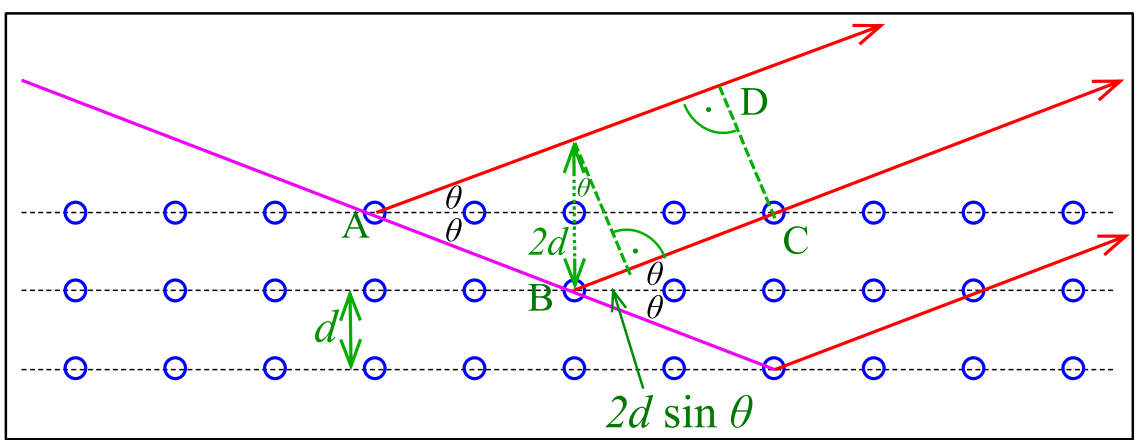

Figure 6. Alternative derivation of Bragg's law with an incident monochromatic wave reflected at lattice points, the path difference between the ray that gets reflected at $\mathrm{A}$ and the one transmitted and reflected at $\mathrm{B}$ needs to be a integral multiple of wavelengths: $(\mathrm{AB}+\mathrm{BC})-(\mathrm{AD})=n \lambda$.

\subsubsection{Von Laue diffraction}

The simple Bragg approach is not easily generalised to include the effects of a basis of atoms (the periodically repeated motif of atoms in each unit cell of a crystal lattice, i.e., the molecule), of electron distribution (in X-ray and electron diffraction) or temperature (thermal displacement of atoms relative to their time-averaged equilibrium position).

Let us start with the usual wave equation for the electric field, which can be derived from the Maxwell equations governing electromagnetic waves such as X-rays (we neglect any other interaction of X-rays with the electron distribution except scattering) [10].

$$
\nabla^{2} E=\frac{1}{c^{2}} \frac{\partial^{2} E}{\partial t^{2}}
$$

For the magnetic field there is a similar wave equation, which shall be neglected in the further discussion of scattering electromagnetic waves for the sake of simplicity. For neutrons we have a similar wave equation derived from the Schrödinger equation:

$$
\nabla^{2} \Psi=-2 i \frac{m}{h} \frac{\partial \Psi}{\partial t}
$$




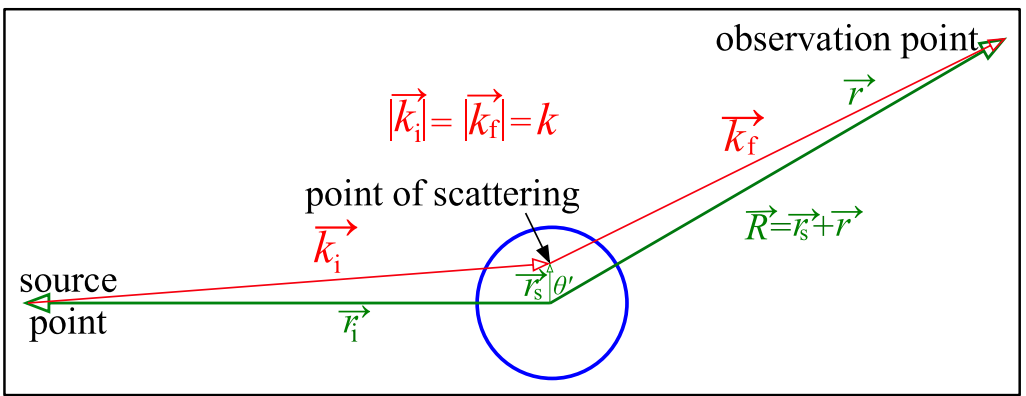

Figure 7. Plane wave scattering with scattering point at $\vec{r}_{\mathrm{s}}$ from origin.

We shall at this stage regard the simpler case of a lattice of point scatterers (with all these points on the lattice knots $^{16}$ ), which is effectively very close to the neutron case, while being too simplifying for $\mathrm{X}$-ray diffraction. The solution of the wave equation for the incident plane wave is

$$
\Psi(\vec{r}, t)=a_{k} e^{i\left(\vec{k} \cdot \vec{r}-\omega_{k} t\right)}
$$

We encounter here for the first time the term "wave vector", $\vec{k}$. Many a physicist is familiar with this vector describing quantitatively and efficiently a wave. Its magnitude, the so-called wavenumber, is inversely proportional to the wavelength and its direction is the direction of the wave's propagation. Unfortunately, one will find two slightly different definitions in the literature, the one used in physics is used here, with the (angular or circular) wavenumber being $k=|\vec{k}|=2 \pi / \lambda$ and $\omega_{k}$ in the wave equation (10) being the angular frequency. In crystallography, one frequently finds the wavenumber being $k^{\prime}=\left|\vec{k}^{\prime}\right|=1 / \lambda$ (in some contexts called spectroscopic wavenumber, $\bar{v}=1 / \lambda$ ) and one uses typically the frequency $v_{k}$ instead of the angular frequency $\omega_{k}$ with $2 \pi v_{k}=\omega_{k}$. Obviously, the same wave equation would then look as follows:

$$
\Psi(\vec{r}, t)=a_{k} e^{i 2 \pi\left(\overrightarrow{k^{\prime}} \cdot \vec{r}-v_{k} t\right)}
$$

The direction of the wave vector is not necessarily identical to the direction of wave propagation, which is the direction of the group velocity, $v_{g}=\partial \omega_{k} / \partial k$, whereas the wave vector points in the direction of phase velocity, $v_{p}=\omega_{k} / k$ (i.e., the speed of light $c$ in the case of photons), which is normal to the surface of constant phase, also called the wave front.

For X-ray diffraction, with the (still) simplified consideration that the scattering electron is found at $\vec{r}_{\mathrm{s}}$ from the origin (the lattice knot, as shown in Fig. 7) one would write in analogy:

$$
E_{i}(\vec{r})=E_{0} e^{i\left(\vec{k}_{i} \cdot \vec{r}_{i}-\omega_{k} t\right)}
$$

The amplitude is $a_{k}$ in the one and $E_{0}$ in the other case, the frequency $\omega=k c$ in the electromagnetic $\mathrm{X}$-ray case. With the wave equation written in spherical coordinates, there is a solution for the spherically scattered wave when neglecting non-dominant terms as we consider only an observation far from the scattering location:

$$
E_{\mathrm{s}}=K_{1} E\left(\vec{r}_{\mathrm{s}}\right)^{i k r} / r
$$

$K_{1}$ is a constant here, and the scattered wave has the same frequency and wavelength as the incident wave (again, we assume elastic scattering at this point, without exchange of energy). Spherically scattered

\footnotetext{
${ }^{16}$ In the treatment of X-ray diffraction one would consider the point scatterer - the electron - being at a distance from the lattice knots.
} 


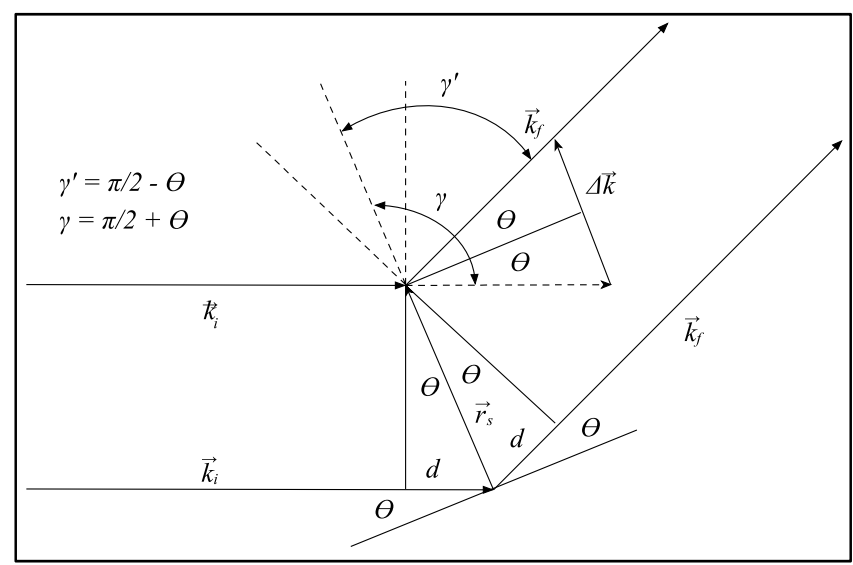

Figure 8. Drawing for simpler discussion of von Laue diffraction approach.

waves are of importance here as the wavelength is greater than the size of the scattering atom (or nucleus, as in the neutron case). With $\vec{r}=\vec{R}-\vec{r}_{\mathrm{s}}$ we obtain for $r_{\mathrm{s}} / R$, i.e., far from the point of scattering

$$
r=R\left(1-r_{\mathrm{s}} /_{R} \cos \theta^{\prime}\right)
$$

With $k r_{\mathrm{s}} \cos \theta \cong \vec{k}_{f} \cdot \vec{r}_{\mathrm{s}}$ one obtains $\vec{k} \vec{r} \cong k R-\vec{k}_{f} \cdot \vec{r}_{\mathrm{s}}$ and thus, from (13) and (12) and the assumption that $r^{-1} \approx R^{-1}$ to sufficient accuracy

$$
E_{\mathrm{s}}=K_{1} E_{0} \frac{e^{i k R}}{R} e^{\mathrm{i}\left(\vec{k}_{i}-\vec{k}_{f}\right) \cdot \vec{r}_{\mathrm{s}} e^{-i \omega_{k} t}}
$$

The phase difference between the wave scattered from the origin and the one scattered from $\vec{r}_{\mathrm{s}}$ is $\left(\vec{k}_{i}-\vec{k}_{f}\right) \cdot \vec{r}_{\mathrm{s}}$ in this approximation. With the scattering vector $\Delta \vec{k}=\vec{k}_{f}-\vec{k}_{i}$, the difference of the wavevectors of the incident and the diffracted beam, we can define the relative scattering amplitude, summed over all scatterers $s$

$$
P=\Sigma_{\mathrm{s}} \mathrm{e}^{-\mathrm{i} \Delta \vec{k} \cdot \vec{r}_{\mathrm{s}}}
$$

The scattered wave can be written as $E_{s c}=F P$ with $|F|=\left|K_{1} E_{0} / R\right|$. It is useful now to come back to a simple geometric consideration. From the central part of Fig. 8 one sees that the path difference between the two beams is $2 d=2 r_{\mathrm{s}} \sin \theta$. With the wave vector's magnitude being $k=2 \pi / \lambda=\left|\vec{k}_{\mathrm{f}}\right|=|\vec{k}|$, the phase difference becomes

$$
\Delta \varphi=\frac{4 \pi}{\lambda} r_{\mathrm{s}} \sin \theta=2 k r_{\mathrm{s}} \sin \theta
$$

Further one reads from Fig. 8:

$$
\Delta \vec{k} \cdot \vec{r}_{\mathrm{s}}=k r_{\mathrm{s}}\left[\cos \left(\frac{\pi}{2}-\theta\right)-\cos \left(\frac{\pi}{2}+\theta\right)\right]=2 k r_{\mathrm{s}} \sin \theta
$$

This is again the phase difference $\Delta \varphi$. For a continuous distribution of scatterers (we are still regarding the case of scattering electromagnetic X-rays at electrons) we obtain with each scatterer scattering proportional to its density $\rho$ (i.e., electron density).

$$
P=\int \mathrm{e}^{-\mathrm{i} \Delta \vec{k} \cdot \vec{r}_{\mathrm{s}}} \rho\left(\vec{r}_{\mathrm{s}}\right) d V
$$

This Eq. (19) means nothing else but the scattering amplitude $P$ being proportional to the Fourier transform (see also Sects. 2.3.2 and 3.2) of the electron density $\rho$. 


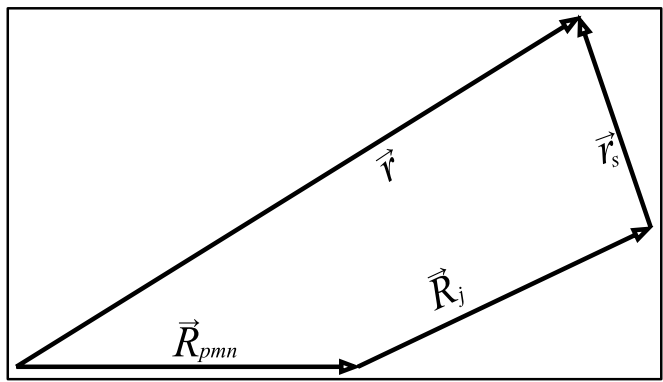

Figure 9. Vector diagram of electron positions.

We assume now the general case of a crystal lattice with a basis of atoms (the periodically repeated "pattern" of atoms, e.g., the molecule(s), in the unit cell), each of those with an electron distribution. The lattice points (the origins of each periodically in three dimensions repeating unit cell) are located at

$$
\vec{R}_{p m n}=p \vec{a}_{1}+m \vec{a}_{2}+n \vec{a}_{3}
$$

Here, $p, m, n$ are integers and $\vec{a}_{1}, \vec{a}_{2}, \vec{a}_{3}$ the fundamental translation vectors of the lattice (i.e., orthogonal to each other in orthorhombic, tetragonal and cubic lattices and identical in magnitude in rhombohedral and cubic lattices). For each $\vec{R}_{p m n}$ there will be a basis of $q$ atoms, with $j=1$ to $q$, at $\vec{R}_{j}=a_{j} \vec{a}_{1}+b_{j} \vec{a}_{2}+c_{j} \vec{a}_{3}$ from the origin of each unit cell at $\vec{R}_{p m n}$. Here, $a_{j}, b_{j}, c_{j}$ are generally non-integer numbers, fractional coordinates of the atoms, by convention, but not necessarily, given as numbers between 0 and 1. With this, as shown in Fig. 9, the electrons are located at $\vec{r}=\vec{R}_{j}+\vec{R}_{p m n}+\vec{r}_{\mathrm{s}}$ and relative to the atoms at $\vec{r}_{\mathrm{s}}=\vec{r}-\vec{R}_{j}-\vec{R}_{p m n}$. The electron density around atom $j$ being $\rho j \overrightarrow{(r)}$, the total electron density becomes

$$
\rho(\vec{r})=\sum_{p m n} \sum_{j=1}^{q} \rho_{j}\left(\vec{r}-\vec{R}_{j}-\vec{R}_{p m n}\right)
$$

With a generalisation of (19) this leads to the scattering amplitude $P$ :

$$
\begin{gathered}
P=\sum_{p m n} \sum_{j=1}^{q} \int \rho_{j}\left(\vec{r}-\vec{R}_{j}-\vec{R}_{p m n}\right) \mathrm{e}^{-\mathrm{i} \Delta \vec{k} \cdot \vec{r}} d V \\
P=\sum_{p m n} e^{-\mathrm{i} \Delta \vec{k} \cdot \vec{R}_{p m n}}\left(\sum_{j=1}^{q} e^{-\mathrm{i} \Delta \vec{k} \cdot \vec{R}_{j}} \int \rho_{j}(\vec{r}) \mathrm{e}^{-\mathrm{i} \Delta \vec{k} \cdot \vec{r}} d V\right)
\end{gathered}
$$

For $N^{3}$ unit cells, the lattice factor separates out and with reciprocal lattice vectors $\vec{G}_{h k l}$ defined below we have:

$$
\sum_{p m n} e^{-\mathrm{i} \Delta \vec{k} \cdot \vec{R}_{p m n}}=N^{3} \delta_{\vec{G}_{h k l}}^{\Delta \vec{k}}
$$

The Kronecker delta $\delta_{\vec{G}_{h k l}}^{\Delta \vec{k}}$ here is defined as follows.

$$
\delta_{\vec{G}_{h k l}}^{\Delta \vec{k}}=0 \text { for } \vec{G}_{h k l} \neq \Delta \vec{k} \wedge \delta_{\vec{G}_{h k l}}^{\Delta \vec{k}}=1 \text { for } \vec{G}_{h k l}=\Delta \vec{k}
$$

This is already von Laue's diffraction condition in a somewhat awkward formulation. 


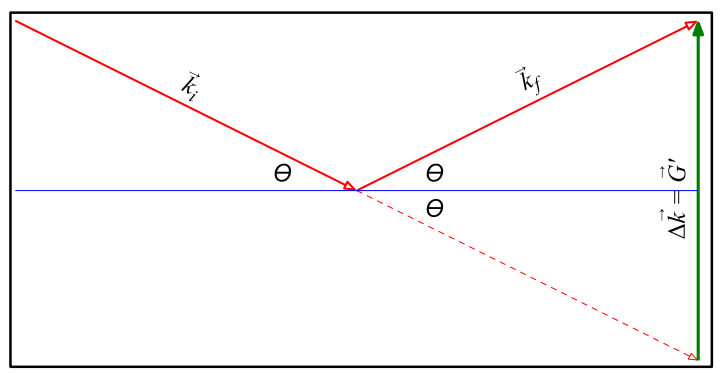

Figure 10. Von Laue diffraction condition: relationship of wave vectors and reciprocal lattice.

\subsubsection{Structure factor}

With this we obtain an expression for the scattering amplitude $P$ by introducing a structure factor $S_{h k l}$ and an atomic form factor $f_{j}$.

$$
\begin{gathered}
P=N^{3} \delta_{\vec{G}_{h k l}^{\Delta \vec{k}} S_{h k l}} \\
S_{h k l}=\sum_{j=1}^{q} e^{-i \vec{G}_{h k l} \cdot \vec{R}_{j}} f_{j}^{h k l} \\
f_{j}^{h k l}=\int \rho_{j}(\vec{r}) \mathrm{e}^{-i \vec{G}_{h k l} \cdot \vec{r}} \mathrm{~d} V
\end{gathered}
$$

For monoatomic lattices, i.e., a basis of atoms of a single element, one can define as well a geometrical structure factor $S_{h k l}^{\prime}=\sum_{j=1}^{q} e^{-i \vec{G}_{h k l} \cdot \vec{R}_{j}}=S_{h k l} / f^{h k l}$, as all form factors are identical.

Let us consider now the special case of a lattice of point scatterers where $f_{j}=1$ and $R_{j}=0$. It is now useful to introduce the important conception of a reciprocal lattice, which will be further elaborated later on (Sect. 2.3). The basis vectors $\vec{b}_{j}$ for this reciprocal lattice are defined by

$$
\vec{a}_{\mathrm{i}} \cdot \vec{b}_{i}=\delta_{i j}
$$

Here, $i$ and $j$ run from 1 to 3 , and $\vec{a}_{j}$ are the basis vectors of the direct lattice and the Kronecker delta $\delta_{i j}$ is defined as follows:

$$
\begin{gathered}
\delta_{i j}=0 \text { for } i \neq j \wedge \delta_{i j}=1 \text { for } i=j \\
\vec{G}_{h k l}=2 \pi\left(h \overrightarrow{b_{1}}+k \overrightarrow{b_{2}}+l \overrightarrow{b_{3}}\right)
\end{gathered}
$$

With the volume of a unit cell in direct space $V=\vec{a}_{1} \cdot\left(\vec{a}_{2} \times \vec{a}_{3}\right)$ we get cyclic relationships of the cross product of two basis vectors to the third reciprocal basis vector:

$$
\vec{b}_{1}=\frac{\vec{a}_{2} \times \vec{a}_{3}}{V}
$$


With this we obtain easily the volume $V^{*}$ of a unit cell in reciprocal space:

$$
V^{*}=\vec{b}_{1} \cdot\left(\vec{b}_{2} \times \vec{b}_{3}\right)=\frac{1}{V}
$$

As the reciprocal basis vectors span three-dimensional space, we can express the scattering vector in terms of them:

$$
\Delta \vec{k}=2 \pi\left(h \overrightarrow{b_{1}}+k \overrightarrow{b_{2}}+l \overrightarrow{b_{3}}\right)
$$

Here, in (34), $h, k, l$ are not necessarily integers. With (20) and (29) we can write the next expression:

$$
\vec{R}_{p m n} \cdot \Delta \vec{k}=2 \pi(p h+m k+n l)
$$

Here, in (35), $p, m, n$ are still integers. From the expression for the scattering amplitude (16), with $\overrightarrow{r_{\mathrm{s}}}=\vec{R}_{p m n}$ (as we wanted to regard a lattice of point scatterers in this subsection), and assuming a lattice of $N^{3}$ atoms, the structure factor becomes as follows:

$$
P=\sum_{p=0}^{N-1} e^{-i 2 \pi p h} \sum_{m=0}^{N-1} e^{-i 2 \pi m k} \sum_{n=0}^{N-1} e^{-i 2 \pi n l}
$$

With the law of geometric progressions this evaluates to the following expression:

$$
|P|^{2}=\left(\frac{\sin ^{2} \pi h N}{\sin ^{2} \pi h}\right)\left(\frac{\sin ^{2} \pi k N}{\sin ^{2} \pi k}\right)\left(\frac{\sin ^{2} \pi l N}{\sin ^{2} \pi l}\right)
$$

For a real lattice we can assume $N \rightarrow \infty$, in which case $|P|$ becomes negligible whenever $h, k, l$ are not integers. If these indices are all integers, each factor becomes $N^{2}$ :

$$
|P|^{2}=N^{6} \delta_{h, k, l}^{\text {integer }}
$$

For a lattice of point scatterers, we have (again) the von Laue condition for diffraction peaks to occur:

$$
\Delta \vec{k}=\vec{k}_{f}-\vec{k}_{i}=\vec{G}_{h k l}=2 \pi\left(h \overrightarrow{b_{1}}+k \overrightarrow{b_{2}}+l \overrightarrow{b_{3}}\right)
$$

In (39), the so-called Miller indices (see Sect. 2.3.1), $h, k, l$, have finally to be integers, in order to have full constructive interference and a diffraction peak, due to (38). Thus, the diffraction peaks determine directly the reciprocal lattice, as presented geometrically in Fig. 10.

Let us now show the equivalence with Bragg's law (7): Let $\vec{G}_{h k l}=n \vec{G}_{h^{\prime} k^{\prime} l^{\prime}}$ with Miller indices $h^{\prime}$, $k^{\prime}, l^{\prime}$ and $\vec{G}_{h^{\prime} k^{\prime} l^{\prime}}$ the shortest vector in the direction of $\vec{G}_{h k l}$. With $\vec{G}_{h k l}$ perpendicular to the plane $(h k l)$ the distance between adjacent planes becomes as follows:

$$
\begin{gathered}
\vec{d}_{h k l}=2 \pi / \vec{G}_{h^{\prime} k^{\prime} l^{\prime}}^{\prime} \\
|\vec{G}|=2 k \sin \theta=n\left|\vec{G}_{h^{\prime} k^{\prime} l^{\prime}}^{\prime}\right|=n \frac{2 \pi}{d_{h k l}}
\end{gathered}
$$

With the angular wavenumber $k=|\vec{k}|=2 \pi / \lambda$ we obtain finally Bragg's law:

$$
n \lambda=2 d_{h k l} \sin \theta \text {. }
$$

\subsubsection{The Debye-Waller factor}

With rising temperature of the scattering crystal, the diffraction peaks have reduced intensity but the diffraction peaks (or lines) do not broaden, they stay sharp. This may come as a surprise to intuition, as 


\section{JDN 21}

thermal motion leads to strong vibration amplitudes in random directions, varying interatomic distances even at room temperature locally by up to $10 \%$. Debye gave the reason for this counterintuitive behaviour. The momentary position $\vec{R}(t)$ of an atom, at rest at $\vec{R}_{j}$, shall contain a time dependent contribution $\vec{u}(t)$, so we have $\vec{R}(t)=\vec{R}_{j}+\vec{u}(t)$. Now we assume independent motion of each atom around its equilibrium position, as in the Einstein model of solid matter, which delivers good results at higher temperatures. The structure factor (27) then writes as follows with the thermal averaging expressed by the brackets " $\langle\cdots\rangle$ ":

$$
S_{h k l}=\sum_{j=1}^{q} e^{i \vec{G}_{h k l} \cdot \vec{R}_{j}}\left\langle e^{-i \vec{G}_{h k l} \cdot \vec{u}}\right\rangle f_{j}^{h k l}
$$

The Taylor series expansion of the exponential function gives the following:

$$
\left\langle e^{i \vec{G}_{h k l} \cdot \vec{u}}\right\rangle=1+i\left\langle\vec{G}_{h k l} \cdot \vec{u}\right\rangle-\frac{1}{2}\left\langle\left(\vec{G}_{h k l} \cdot \vec{u}\right)^{2}\right\rangle-\frac{i}{6}\left\langle\left(\vec{G}_{h k l} \cdot \vec{u}\right)^{3}\right\rangle+\frac{1}{24}\left\langle\left(\vec{G}_{h k l} \cdot \vec{u}\right)^{4}\right\rangle+\cdots
$$

The random thermal displacements $\vec{u}$ are not correlated with the direction of $\vec{G}_{h k l}$ and therefore $\left\langle\vec{G}_{h k l} \cdot \vec{u}\right\rangle=0$, i.e., all odd terms disappear. For the third, squared, term in the series we further have the following relation:

$$
\left\langle\left(\vec{G}_{h k l} \cdot \vec{u}\right)^{2}\right\rangle=G_{h k l}{ }^{2}\left\langle u^{2}\right\rangle\left\langle\cos ^{2} \theta\right\rangle=\frac{1}{3}\left\langle u^{2}\right\rangle G_{h k l}{ }^{2}
$$

The factor $\frac{1}{3}$ is the geometrical average of $\cos ^{2} \theta$ over a sphere. Now let us consider another Taylor series expansion:

$$
e^{-\frac{1}{6}\left\langle u^{2}\right\rangle G_{h k l^{2}}}=1-\frac{1}{6}\left\langle u^{2}\right\rangle G_{h k l}{ }^{2}+e-\frac{1}{72}\left\langle u^{2}\right\rangle^{2} G_{h k l}{ }^{4}+\cdots
$$

With (45) and was has been said before, the first two terms of (46) become identical to the first three terms of (44). For an harmonic oscillator even all terms of (44) and (46) are identical. With this fact, entered in (43), we obtain for the scattering intensity $I$ - the squared of the scattering amplitude $P$ as in (26) - the following exponential correction factor - the Debye-Waller factor - to a scattering intensity $I_{0}$ of a rigid lattice.

$$
I=I_{0} e^{-\frac{1}{3}\left\langle u^{2}\right\rangle G_{h k l^{2}}}
$$

Here, $\left\langle u^{2}\right\rangle$ is the mean squared displacement of an atom. The mean thermal potential energy $\langle U\rangle$ of a classical harmonic oscillator in three dimensions is $\frac{3}{2} k_{B} T$ with the Boltzmann constant $k_{B}$ and the temperature $T$ :

$$
\langle U\rangle=\frac{1}{2} C\left\langle u^{2}\right\rangle=\frac{1}{2} M \omega^{2}\left\langle u^{2}\right\rangle=\frac{3}{2} k_{B} T
$$

$C$ is a force constant, $M$ the atom's mass and $\omega$ the frequency of the oscillator. With this we obtain for the scattering intensity of a diffraction peak an expression, which is a good approximation at higher temperatures:

$$
I=I_{0} e^{-\frac{3}{2} k_{B} T G_{h k l^{2}} / M \omega^{2}}
$$

In the model of independent harmonic oscillators there is a zero-point energy of $\frac{3}{2} \hbar \omega$, half of it potential energy $\langle U\rangle$, as there is still motion $\left(\left\langle u^{2}\right\rangle \neq 0\right)$ at $T=0$. With this, (48) leads to $\left\langle u^{2}\right\rangle=3 \hbar / 2 M \omega$ and $I=I_{0}^{-\hbar G_{h k l} / 2 M \omega}$ at $T=0$. By putting some crude estimates, $G_{h k l}=10^{11} \mathrm{~m}^{-1}, \omega=10^{14} \mathrm{~s}^{-1}$ and $M=10^{-25} \mathrm{~kg}$ one obtains an intensity $I$ of about $95 \%$ of $I_{0}$ : At $T=0 \mathrm{~K}$, about $95 \%$ of the intensity may be scattered elastically at the position of the Bragg peak, and 5\% inelastically, near the position of the Bragg peak as so-called thermal diffuse scattering (TDS). For us, interested mainly in the elastic 
part of scattering, it may be important to be able to deconvolute the elastic scattering intensity from the TDS underlying the detected intensity at the position of the Bragg peak.

Another approach leads to a slightly different result as in (47): The Debye-Waller factor is nothing else but the Fourier transform of the probability density function (p.d.f.), $p(u)$, of an atom (its thermal smearing). For a Boltzmann distribution of simple harmonic oscillators in thermal equilibrium at a temperature $T, p(u)$ is a Gaussian function of the displacement $u$ (we limit ourselves here to the isotropic case).

$$
p(u)=\left(2 \pi\left\langle u^{2}\right\rangle\right)^{\frac{3}{2}} e^{-\frac{u^{2}}{2\left\langle u^{2}\right\rangle}}
$$

The Fourier transform of this p.d.f. results in another Debye-Waller factor to "correct" the elastically scattered intensity:

$$
I=I_{0} e^{-\left\langle u^{2}\right\rangle G_{h k l^{2}}}
$$

One calls the whole exponential factor (Gaussian) Debye-Waller factor $e^{-\left\langle u^{2}\right\rangle G_{h k l^{2}}}=e^{-2 W}$, which is nothing else than the fraction of elastic scattering as compared to inelastic scattering at a given $G_{h k l}$, and half of the (negative) exponent the Debye-Waller function $W=\frac{1}{2}\left\langle\left(\vec{G}_{h k l} \cdot \vec{u}\right)^{2}\right\rangle=\frac{1}{2}\left\langle u^{2}\right\rangle G_{h k l}{ }^{2}$. The latter is a product of the reciprocal lattice vector and a factor proportional to the several conventions of an atomic displacement parameter, frequently and wrongly called "temperature factor" 17 , as it depends (approximately linearly) on temperature, see (49). Two conventions dominate: the mean squared atomic displacement $U=\left\langle u^{2}\right\rangle$ and the surface of the sphere enclosing the likely place of the atom, i.e., with a radius $\sqrt{ }\left\langle u^{2}\right\rangle, B=8 \pi^{2} U$ (thus, $W=B \sin ^{2} \theta / \lambda^{2}$ ). The latter has an order of magnitude of slightly less than $1 \AA$ for tightly bound atoms, e.g., in a metal oxide, or slightly more for molecular compounds at room temperature. If the atomic displacement anisotropic and described by a tensor, i.e., dependent on the direction of the lattice vector, we obtain the anisotropic Debye-Waller factor, otherwise, for isotropic displacements, the atomic isotropic one, or presuming all atoms having the same isotropic displacement, the overall isotropic one.

$\mathrm{X}$-ray diffraction, and in particular X-ray powder diffraction, rarely delivers sufficient information to determine anisotropic atomic displacement parameters for all atoms, whereas neutron diffraction, even neutron powder diffraction in many cases, delivers anisotropic atomic displacement parameters more easily.

One may be tempted using (49) to exploit together with the zero-point energy the temperaturedependency of atomic displacement factors, which can be obtained from (elastic) diffraction experiments in order to obtain "spectroscopic" information, i.e., the frequency of the harmonic oscillator $\omega$, usually reserved for inelastic scattering experiments, based on the harmonic approximation in the theory of lattice dynamics, and indeed this is not impossible. However, one has to keep in mind, that there is also a temperature-independent contribution of static displacive disorder (hence the wrongness of the term temperature factor) to be added to the zero-point energy at $T=0$. To illustrate this, one may take the example of a noble gas atom inside the (too) large cage of a gas hydrate clathrate. In average, the atom occupies the central position of the "rattling cage", but close to $T=0$, it will more likely occupy one of several positions not exactly in the centre but closer to the cage faces, due to the van-der-Waals attraction to the cage-building water molecules.

17 One finds in the literature the term temperature factor as the square root of the Debye-Waller factor, i.e., in the same way the Debye-Waller factor $e^{-2 W}$ corrects the scattered intensity for the effects of thermal motion, the temperature factor $T=e^{-W}$ does this for the structure factor. 


\section{Crystallography}

In this section we shall present some basics in crystallography. Everything we have done yet is to throw some basic notions about the diffraction method in general. We need this method to elucidate the structure of most condensed matter, of crystalline matter. Despite appearance, most solid matter is indeed crystalline, or, rather, polycrystalline. And for the remaining materials, which are not crystalline, but amorphous, such as glasses, or liquids, we can apply the same method as well in a certain sense to understand their local structure, only that we won't observe the same sharp Bragg diffraction peaks as described before. Unfortunately, the description of diffraction experiments on these disordered materials explode the scope of this chapter.

Crystals fascinated humanity since ever. The antique Greeks, for instance, considered those beautiful quartz crystals frequently found in the mountains as ice, which became eternal due to the long time they stayed at cold weather. This wrong believing is in fact the origin of the word: " $\kappa \rho \sigma \tau \alpha \lambda \lambda o \varsigma$ " has a meaning close to "ice" and was used for rock crystal and crystals of similar appearance. In 1669, Steno gave the law of constant angles between similar crystal faces, and with this the idea germinated that some underlying microscopic symmetry must be inherent in crystals.

\subsection{Crystal symmetry}

The so-obvious symmetries of crystals determine many of their properties and simplify many calculations. Ideal crystals are real crystals without defects and boundaries, i.e., they are infinite. They exhibit several types of symmetry:

\subsubsection{Group theory}

The most appropriate formalism to discuss the symmetry properties of solids is the group theory. For crystal symmetry only finite groups are of interest. We will introduce some basics to use the crystal symmetry operations as examples of groups and to help us organising in our mind the symmetries present in crystals.

There are two basic ingredients of any group: a set of elements $G=\left\{g_{1}, g_{2}, \ldots\right\}$ and an operation (*) for their combination. Four rules must be satisfied:

1. Closure: If $g_{\mathrm{i}}$ and $g_{\mathrm{j}}$ are elements of $G$, then $g_{\mathrm{i}} * g_{\mathrm{j}} \in G$.

2. Associative law: If $g_{\mathrm{i}}, g_{\mathrm{j}}$ and $g_{\mathrm{k}}$ are elements of $G$, then $\left(g_{\mathrm{i}} * g_{\mathrm{j}}\right) * g_{\mathrm{k}}=g_{\mathrm{i}} *\left(g_{\mathrm{j}} * g_{k}\right)$

3. Existence of an identity: There must exist a $g_{\mathrm{e}} \in G$ with $g_{\mathrm{j}} * g_{\mathrm{e}}=g_{\mathrm{e}} * g_{\mathrm{i}}=g_{\mathrm{i}}$

4. Existence of an inverse: For each $g_{\mathrm{i}} \in G$ there is a $g_{\mathrm{i}}^{-1} \in G$ with $g_{\mathrm{j}} * g_{\mathrm{i}}^{-1}=g_{\mathrm{i}}^{-1} * g_{\mathrm{i}}=g_{\mathrm{e}}$.

A proper subgroup of $G$ is a group $A$ contained in $G$, not identical to the identity $E$, a trivial group of order one. Two operations belong to the same class if they perform the same sort of geometrical operation. A cyclic group can be generated by a single generator element $g$. For such a group of order $N$ we have $g^{N}=E$. The order of a group element is the smallest power to which the element can be raised and still yield $E$, thus, $N$ is the order of the generator $g$ of the latter cyclic group of order $N$.

\subsubsection{Translation}

Let $m_{1}, m_{2}, m_{3}$ be arbitrary integers. A crystal is translationally symmetric - or periodic - if there are three linearly independent vectors $\left(\vec{a}_{1}, \vec{a}_{2}, \vec{a}_{3}\right)$ such that a translation by $m_{1} \vec{a}_{1}+m_{2} \vec{a}_{2}+m_{3} \vec{a}_{3}$ brings one back to an equivalent point in the crystal. The $\vec{a}_{\text {i }}$ are called basis vectors, they do not need to be orthogonal. A set $\left(\vec{a}_{1}, \vec{a}_{2}, \vec{a}_{3}\right)$ is not unique, any linear combination with integer coefficients gives another set. By parallel extension, the $\vec{a}_{\mathrm{i}}$ form a parallelepiped with a volume $V=\vec{a}_{2} \cdot\left(\vec{a}_{2} \times \vec{a}_{3}\right)$, called 
unit cell. These fill by stacking all space and corresponding points in different unit cells are equivalent. The smallest possible unit cells satisfying the latter conditions are called primitive cells (which are not necessarily unique). The corresponding basis vectors are then called primitive translations. The set of all translations $T=m_{1} \vec{a}_{1}+m_{2} \vec{a}_{2}+m_{3} \vec{a}_{3}$ forms a group of infinite order.

The symmetry operations of a crystal are all those operations bringing the crystal back onto itself. Translations are one example for this, but there are others, like rotations and reflections:

\subsubsection{Point symmetry operations}

Identity. Although this is trivial, it is a necessary, omnipresent and fully counted point symmetry operation in the description of crystal symmetry. One uses the symbol $E$ or

Rotation axis. We have an axis of symmetry of the $n^{\text {th }}$ order, if a rotation of $2 \pi / n$ about the axis maps the crystal back onto itself. The symbol $C_{n}$ is frequently used for this (notation proposed by Schönflies in 1891), note that $\left(C_{n}\right)^{n}=C_{1}=E$, with the identity element $E$. In another notation, named after Hermann (1928) and Maugin (1931), one uses simply the number $n$ as symbol, i.e., 2, 3, 4 and 6 for $C_{2}$, $C_{3}, C_{4}$ and $C_{6}$, respectively, the only rotation axes one will find in crystals. One notes that 5-fold or 7and more-fold axes are not among the examples chosen, and that is for a good reason, as we will see soon.

Reflection plane. A unit cell is mapped to itself when reflected in a plane of reflection symmetry. The operation is often called $\sigma$, with $\sigma^{2}=E$. The Hermann-Maugin symbol is $m$ (or $\overline{2}$ ).

Rotary reflection axis. We have a rotary reflection (or rotoreflection) axis of $n^{\text {th }}$ order, if a rotation of $2 \pi / n$ about the axis and a simultaneous reflection through a plane normal to the axis maps the crystal back onto itself. The symbol $S_{n}$ is frequently used for this. If a reflection in a plane perpendicular to this axis is called $\sigma_{\mathrm{h}}$, then we have $S_{n}=C_{n} \sigma_{\mathrm{h}}$. A reflection plane is thus a first-order rotary reflection: $S_{1}=C_{1} \sigma_{\mathrm{h}}=E \sigma_{\mathrm{h}}=\sigma_{\mathrm{h}}$. The Herman-Maugin symbols for $S_{3}, S_{4}$ and $S_{6}$ are $\overline{6}, \overline{4}$ and $\overline{3}$, respectively, for the relevant rotary reflection axes in crystals.

Inversion centre. If we have any function $f(x, y, z)$ of Cartesian coordinates $(x, y, z)$, then we have an inversion I through the origin if $I[f(x, y, z)]=f(-x,-y,-z)$. A second-order rotary reflection is the same as an inversion: $S_{2}=C_{2} \sigma_{\mathrm{h}}=I$. The Hermann-Maugin symbol is $\overline{1}$. One has to explain the seemingly discrepancy of the two notations for rotary reflections, when it comes to first, second, third and sixth order: The same symmetry operation can be obtained by combining a rotation with an inversion (instead of a reflection). This rotary inversion (or rotoinversion) is underlying the Herman-Maugin symbols, and we have $\overline{1}=S_{2}, \overline{2}=m=S_{1}, \overline{3}=S_{6}$ and $\overline{6}=S_{3}$. The multiplicities of rotary inversion and rotary reflection do not coincide if not divisible by four. An inversion centre is automatically present for each rotary inversion of an odd order, and a reflection plane is present for all rotary inversions of even order, but not divisible by four.

\subsubsection{Translation symmetry operations}

On top of these, a crystal may contain symmetry elements that are not simply group products of the point symmetry elements.

Screw axis. The symmetry operation, which maps each point on an equivalent point by simultaneously rotating by $2 \pi / n$ and translating by $d$, is a screw axis. Crystallographically allowed screw axes show a displacement parallel to the rotation axis. For such an $n$-fold axis only displacements 


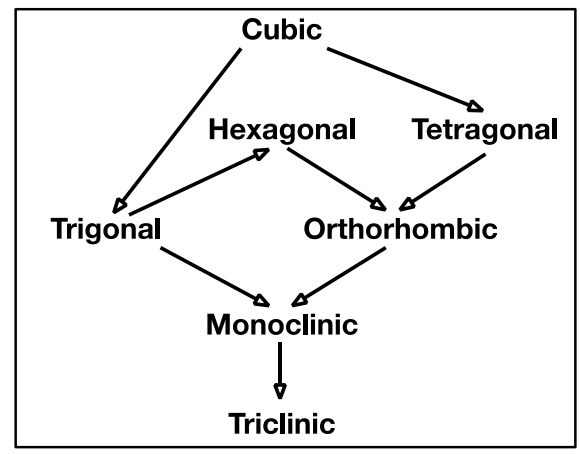

Figure 11. Hierarchy of crystal systems; each Bravais lattice point group contains all those that can be reached from it by moving in the directions of the arrows.

of $d=p a / n$ are possible, with $a$ the smallest period in the axis direction (i.e., the lattice constant) and $p \in\{1,2, \cdots, n-1\}$. A zero-fold screw axis $(p=0)$ corresponds to the simple rotation axis $n$. Possible screw axes $n_{p}$ are thus (Hermann-Maugin symbols): $2_{1}, 3_{1}, 3_{2}, 4_{1}, 4_{2}, 4_{3}, 6_{1}, 6_{2}, 6_{3}, 6_{4}$ and $6_{5}$.

Glide plane. The symmetry operation, which maps each point on an equivalent point by simultaneously reflecting through the plane and translates by $d$, is a glide plane. In general, the displacement is parallel to the plane. With $a$ being the smallest period in translation direction, then only glide-reflections are crystallographically possible, where $2 d=a$.

\subsection{Crystal structure}

A set of points defined by $m_{1} \vec{a}_{1}+m_{2} \vec{a}_{2}+m_{3} \vec{a}_{3}$ is called a lattice, a three-dimensional net-like structure. If one places at each point an identical collection or basis of atoms, the resulting structure is called a crystal structure. In other words, a crystal is the convolution of a basis and a lattice. The basis will have no symmetry not already contained in the lattice. The lattice-defining points do not necessary coincide with the location of atoms.

\subsubsection{Thirty-two point groups}

Point groups are collections of crystal symmetry operations (i.e., rotation and rotary reflection axes) that form a group and leave one point fixed (thus its name). From the above, the point group of a basis must be also a valid point group of the lattice.

Only 32 point groups are compatible with infinite periodic three-dimensional dense packing, as fivefold, seven- or more-fold symmetry elements are not possible, unless one regards individual molecules or a quasi-crystalline lattice [11]. As already implied when presenting the symmetry operations, a crystal can only have one-, two-, three-, four- and six-fold symmetry axes. For a mathematical proof of this theorem, see section 1.2 .3 of [10].

\subsubsection{Fourteen Bravais lattices}

A Bravais lattice or lattice type is an infinite array of discrete points with an arrangement and orientation that appears exactly the same, from whichever of the points the array is viewed [12]. From the point of symmetry, a Bravais lattice is characterized by the specification of all operations that take the lattice to itself, which form the so-called space group of the Bravais lattice. The latter contains only translations 
through Bravais lattice vectors, operations that leave a particular point of the lattice fixed, and operations constructed by successive applications of the previous two types.

There are 14 Bravais lattices. Crystals can have only these 14 possible different parallelepiped networks of lattice points. Formulated differently, the requirement that a lattice must be invariant under one of the 32 point-groups leads to 14 symmetrically specialized lattices types. The general lattice is triclinic. In two dimensions, there are five Bravais lattices ${ }^{19}$.

The volume of space that just fills out all space without overlapping or leaving voids when translated through all vectors in a Bravais lattice is called a primitive unit cell or primitive cell of the lattice. Such a primitive cell must contain precisely one lattice point. Its volume $v$ is $v=1 / n$ with $n$ being the density of points in the lattice.

One can fill space in a similar way with - not necessarily primitive - conventional unit cells or simply unit cells. Such a cell is a region that fills space without overlap and voids when translated through a subset of Bravais lattice vectors. It is generally chosen bigger than the primitive cell, e.g., a body-centred cubic lattice is described with a cubic unit cell, twice as large as the primitive cell, i.e., containing two lattice points. The numbers specifying the size of a unit cell are called lattice constants, e.g., the single number $a$ for cubic crystals.

\subsubsection{Seven crystal systems}

All 14 lattice-types can be attributed to one of seven possible crystal systems. If one considers not the complete space group of a Bravais lattice, but only those operations that leave a particular point fixed, one obtains a subset of the full symmetry group of the Bravais lattice, the point group of the latter. There are only seven distinct point groups a Bravais lattice can have. One can derive the Bravais lattices from each crystal system by adding translation operations, which are body centring $(I)$, face centring $(F)$ on all faces and base centring $(A, B$, or $C$, all equivalent) on only one (base) face. However, only the orthorhombic crystal system supports all three types of centring on top or the primitive $(P)$ lattice without centring. This is for one of two reasons, either, the centring is not compatible with the symmetry (e.g., the base centring of a cubic lattice breaks the cubic symmetry, brings it to tetragonal), or, the centring results in another possible lattice of smaller unit cell volume (e.g., the base centring of a tetragonal lattice results in a primitive lattice with a lattice corresponding to half of the diagonal of the larger base plane).

The cubic system has the highest symmetry and contains three Bravais lattices; the primitive one (simple cubic), the body- and the face centred ones. By pulling or pushing on two opposite faces of a cube, one obtains a rectangular prism with square base, the tetragonal system, where we have two Bravais lattices, a body-centred beside the primitive one. Continuing deformations on the remaining square face, one will distort to the orthorhombic system, with all faces still mutually perpendicular but not squared. This system has all four possible Bravais lattices to offer, the primitive, the body- and face-centred one and the base-centred one. By distorting the rectangular faces of this perpendicular to one axis one obtains a parallelogram corresponding to the monoclinic system. Two Bravais lattices are possible here, the primitive and a base-centred one. The final distortion leads to the triclinic system, with only a primitive Bravais lattice, all axes are not equal in angle and length.

The trigonal system can be derived from the cube by stretching a cube along one body diagonal (the three-fold symmetry axis). Only the primitive Bravais lattice is possible. Derived like this, one would rather call it the rhombohedral system with three primitive vectors of equal length and equal angles with one another. However, there is a trigonal setting possible as well, corresponding to the hexagonal one, with a base made from two equal primitive vectors at an angle of $120^{\circ}$, and a third primitive vector of different length perpendicular to this base. Distortion of the trigonal system brings us to the monoclinic one. Finally, the hexagonal system, of higher symmetry as compared to the trigonal one, is not directly derived from the cube; it is a right prism with a regular hexagon as base. Only the primitive Bravais 
JDN 21

Table 2. 61 out of 73 symmorphic space groups (plus 5 trigonal and 7 with alternative orientation).

\begin{tabular}{|c|c|c|c|c|c|c|c|c|}
\hline $\begin{array}{l}\text { Crystal } \\
\text { system }\end{array}$ & \multicolumn{4}{|c|}{ Centring } & $\begin{array}{l}\text { point } \\
\text { groups }\end{array}$ & $\begin{array}{l}\text { Bravais } \\
\text { lattices }\end{array}$ & $\begin{array}{l}\text { point } \\
\text { groups }\end{array}$ & $\begin{array}{l}\text { space } \\
\text { groups }\end{array}$ \\
\hline triclinic & $P$ & & & & -1 & 1 & 2 & 2 \\
\hline \multirow[b]{3}{*}{ monoclinic } & \multirow[b]{3}{*}{$\mathrm{P}$} & \multirow[b]{3}{*}{$\mathrm{C}$} & & & 2 & \multirow[b]{3}{*}{2} & \multirow[b]{3}{*}{3} & \multirow[b]{3}{*}{6} \\
\hline & & & & & $\mathrm{m}$ & & & \\
\hline & & & & & $2 / \mathrm{m}$ & & & \\
\hline \multirow[b]{3}{*}{ orthorhombic } & \multirow[b]{3}{*}{$\mathrm{P}$} & \multirow[b]{3}{*}{$\mathrm{C}$} & \multirow[b]{3}{*}{ I } & \multirow[b]{3}{*}{$\mathrm{F}$} & 222 & \multirow[b]{3}{*}{4} & \multirow[b]{3}{*}{3} & \multirow[b]{3}{*}{12} \\
\hline & & & & & $\mathrm{mm} 2$ & & & \\
\hline & & & & & $\mathrm{mmm}$ & & & \\
\hline \multirow[b]{7}{*}{ tetragonal } & \multirow[b]{7}{*}{$\mathrm{P}$} & & \multirow{7}{*}{\multicolumn{2}{|c|}{ I }} & -4 & \multirow[b]{7}{*}{2} & \multirow[b]{7}{*}{7} & \multirow[b]{7}{*}{14} \\
\hline & & & & & 4 & & & \\
\hline & & & & & $-42 m$ & & & \\
\hline & & & & & 422 & & & \\
\hline & & & & & $4 / \mathrm{m}$ & & & \\
\hline & & & & & $4 \mathrm{~mm}$ & & & \\
\hline & & & & & $4 / \mathrm{mmm}$ & & & \\
\hline \multirow[b]{5}{*}{ trigonal } & \multirow[b]{5}{*}{$\mathrm{P}$} & & & & 3 & \multirow[b]{5}{*}{1} & \multirow[b]{5}{*}{5} & \multirow[b]{5}{*}{5} \\
\hline & & & & & 32 & & & \\
\hline & & & & & -3 & & & \\
\hline & & & & & $-3 m$ & & & \\
\hline & & & & & $3 \mathrm{~m}$ & & & \\
\hline \multirow[b]{7}{*}{ hexagonal } & \multirow[b]{7}{*}{$\mathrm{P}$} & & & & -6 & & & \\
\hline & & & & & 6 & & & \\
\hline & & & & & $-6 m^{2}$ & & & \\
\hline & & & & & 622 & & & \\
\hline & & & & & $6 / \mathrm{m}$ & & & \\
\hline & & & & & $6 / \mathrm{mm}$ & & & \\
\hline & & & & & $6 / \mathrm{mmm}$ & 1 & 7 & 7 \\
\hline & & & & & 23 & & & \\
\hline & & & & & $-43 m$ & & & \\
\hline & & & & & 432 & & & \\
\hline & & & & & $m-3$ & & & \\
\hline cubic & $\mathrm{P}$ & & I & $\mathrm{F}$ & $m-3 m$ & 3 & 5 & 15 \\
\hline 7 & & & & & & 14 & 32 & 61 \\
\hline
\end{tabular}

lattice is possible. The primitive vectors are set like the trigonal setting of the trigonal system (thus, the relationship to this system). There is a relationship to the $C$-centred orthorhombic Bravais lattice: the latter can be obtained by distorting the hexagonal prism perpendicular to one side of it.

\subsubsection{Two hundred thirty space groups}

For each crystal system, one can combine the different point groups (of equal or less symmetry than the seven point groups of the Bravais lattices) with the different Bravais lattices and one will find 61 space groups, just by multiplying, as shown in Table 2. Five extra space groups add, which is related to the particular rhombohedral setting (" $R$ ") in the five trigonal point groups: they arise when placing an object 
with trigonal symmetry in a hexagonal lattice. Indeed, the trigonal crystal system is an unusual one and one will find in some crystallography contexts, emphasising point symmetry rather than translational symmetry ${ }^{18}$, only six crystal systems, because trigonal point groups can characterise a crystal structure with hexagonal Bravais lattice. Another seven space groups arise from the fact that an object with the symmetry of a certain point groups, i.e., $\mathrm{mm} 2, \overline{4} \mathrm{~m}, 32,3 \mathrm{~m}, \overline{3} \mathrm{~m}$ and $\overline{6} \mathrm{~m}$ can be oriented in more than one way in a given, respectively, orthorhombic, tetragonal, trigonal, or hexagonal Bravais lattice. Altogether, this gives 73 symmorphic space groups. The remaining 157 non-symmorphic space groups arise from the addition of translational symmetry operations, screw axes and glide planes.

\subsection{Reciprocal lattice}

Bragg diffraction does not measure atomic distances in real space. The (angular) distances actually measured are rather inversely proportional to distances $d$ of lattice planes. Further, the $n$ in Bragg's law, Eq. (7), means measuring the number of wavelengths fitting between two lattice planes, i.e., rows of atoms. This number is a reciprocal distance.

The conception of reciprocal lattice is of central importance in most studies of periodic structures. Various avenues lead to it, thus as the theory of crystal diffraction, as already used above in Sect. 1.4.2, but also the abstract study of functions with the periodicity of a Bravais lattice or the law of momentum conservation when the translational symmetry of free space is reduced to that of a periodic potential [12].

A set of points $\vec{R}$ constitutes a Bravais lattice, and $e^{i \vec{k} \cdot \vec{r}}$ a plane wave. For a general wave vector $\vec{k}$ the plane wave has not the periodicity of the Bravais lattice, but only for certain ones. The reciprocal lattice is the set of all wave vectors $\vec{K}$ yielding plane waves with the periodicity of a given Bravais lattice. A vector $\vec{K}$ belongs to the reciprocal lattice of a Bravais lattice of points $\vec{R}$, if for any $\vec{r}$ and for any $\vec{R}$ in the Bravais lattice the following relation holds:

$$
\mathrm{e}^{\mathrm{i} \vec{K} \cdot(\vec{r}+\vec{R})}=\mathrm{e}^{\mathrm{i} \vec{K} \cdot \vec{r}}
$$

Now we can characterize the reciprocal lattice as the set of wave vectors $\vec{K}$ that satisfy the following condition for all $\vec{R}$ in the Bravais lattice:

$$
\mathrm{e}^{\mathrm{i} \vec{K} \cdot \vec{R}}=1
$$

There are some important properties of a reciprocal lattice, which is derived from a direct Bravais lattice. The reciprocal lattice is a Bravais lattice itself, and the reciprocal lattice of a reciprocal lattice is the original direct lattice.

\subsubsection{Miller indices}

A lattice plane is defined as any plane containing at least three non-collinear Bravais lattice points. Because of the translational symmetry any such plane has an infinite number of lattice points forming a two-dimensional Bravais lattice ${ }^{19}$ within the plane.

To describe the orientation of these lattice planes, one uses quite generally vectors normal to the planes. Reciprocal lattice vectors are normal to any family of lattice planes, and the shortest such vector identifies the planes in a unique way: The Miller indices of a lattice plane are the coordinates of the

\footnotetext{
18 This emphasis on point symmetry comes historically from the bases of crystallography in the morphology, which allowed the determination of point symmetry before the advent of diffraction.

19 There are five two-dimensional Bravais lattices: oblique (related to monoclinic and triclinic: two different axes not normal on each other), rectangular (related to orthorhombic), rhombic (= centered rectangular, related to base centered orthorhombic), hexagonal and square (related to tetragonal).
} 


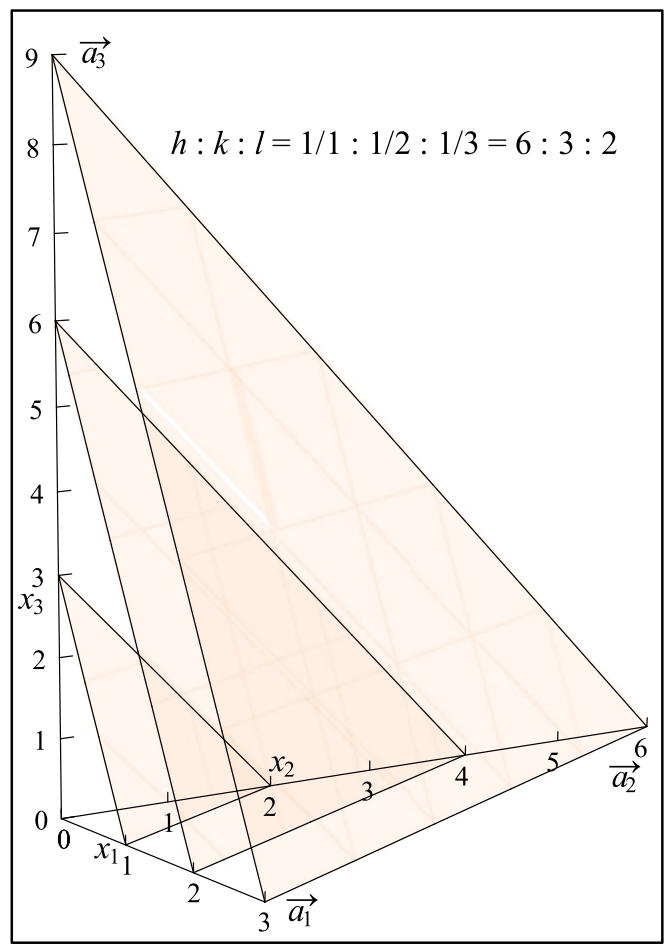

Figure 12. A family of lattice planes $(h k l)=(632)$, one intercepting the basis vectors $\vec{a}_{1}$ at $x_{\mathrm{i}}$. The reciprocals of $x_{\mathrm{i}}$, multiplied by the greatest common divisor (6) deliver $h, k, l$, the Miller indices.

shortest reciprocal lattice vector normal to it. Thus, a plane with Miller indices $h, k, l$, is normal to the reciprocal lattice vector $h \overrightarrow{b_{1}}+k \overrightarrow{b_{2}}+l \overrightarrow{b_{3}}$. The Miller indices are integers and any reciprocal lattice vector is a linear combination of three primitive vectors with integral coefficients. The three Miller indices cannot have a common factor, representing the shortest vector.

Miller indices of a plane have a geometrical interpretation in the direct lattice, offering an alternative of defining them: since the plane with indices $h, k, l$ is perpendicular to the reciprocal lattice vector $\vec{K}=h \overrightarrow{b_{1}}+k \overrightarrow{b_{2}}+l \overrightarrow{b_{3}}$, it will be contained in the plane $\vec{K} \cdot \vec{r}=A$ with constant $A$. This plane intersects the aces determined buy the direct lattice primitive vectors $\overrightarrow{a_{1}}$ at the points $x_{1} \overrightarrow{a_{1}}, x_{2} \overrightarrow{a_{2}}$, and $x_{3} \overrightarrow{a_{3}}$, where $x_{I}$ are determined by the condition that $x_{\mathrm{i}} \cdot \overrightarrow{a_{1}}$ satisfy the equation of the plane $\vec{K} \cdot\left(x_{1} \overrightarrow{a_{1}}\right)=A$. With $\vec{K} \cdot \overrightarrow{a_{1}}=2 \pi h, \vec{K} \cdot \overrightarrow{a_{2}}=2 \pi k$ and $\vec{K} \cdot \overrightarrow{a_{3}}=2 \pi l$ it follows

$$
x_{1}=\frac{A}{2 \pi h}, \quad x_{2}=\frac{A}{2 \pi k}, \quad x_{3}=\frac{A}{2 \pi l}
$$

The intercepts with the crystal axes of a lattice plane are inversely proportional to the Miller indices. In crystallography, the Miller indices are defined to be a set of integers with no common factors and inversely proportional to the intercepts of the crystal plane along the crystal axes:

$$
h: k: l=\frac{1}{x_{1}}: \frac{1}{x_{2}}: \frac{1}{x_{3}}
$$

Lattice planed are specified by their Miller indices in parentheses: $(h, k, l)$. Commas and spaces are frequently left out $(h k l)$, when possible without confusion, and negative signs replaced by a bar above the index, thus $(-h,-k,-l)=(\bar{h} \bar{k} \bar{l})$. 
A similar convention is used to specify directions in direct space, where confusion with Miller indices (planes in reciprocal space) is avoided by using square brackets instead of parentheses. Thus, e.g., the body diagonal of a cubic lattice lies in the [111] direction (which is symmetry-equivalent to

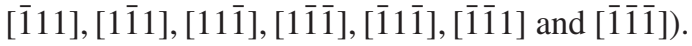

One uses $\{h k l\}$ to refer the planes $(h k l)$ and all those that are symmetry-equivalent. Taking the above case, the eight different lattice planes perpendicular to the diagonal of a cubic lattice would be referred to as $\{111\}$. Similarly, symmetry-equivalent directions $[h k l]$ are referred to, collectively, as $\langle h k l\rangle$, e.g., $\langle 111\rangle$, in the case of the eight equivalent diagonals in a cubic lattice.

\subsubsection{Fourier transformation}

As already mentioned, a crystal $C(\vec{r})$ can be described as the convolution of a basis of atoms $B(\vec{r})$ and a lattice $L(\vec{r}): C(\vec{r})=B(\vec{r}) * L(\vec{r})$. We shall now come back to a diffraction experiment on a crystal. While measuring the diffraction angles will tell us about the metric characteristics of the crystal, its crystal system, the size of the unit cell, the diffracted intensities will allow us to learn about the structure of the basis, the content of the asymmetric unit. The diffracted amplitude corresponds to a Fourier transform of the diffusing object, the crystal, convolution of basis and lattice:

$$
C(\vec{h})=\int_{-\infty}^{\infty} C(\vec{r}) e^{-2 \pi i \vec{r} \cdot \vec{h}} d \vec{r}=\int_{-\infty}^{\infty}[L(\vec{r}) * B(\vec{r})] e^{-2 \pi i \vec{r} \cdot \vec{h}} d \vec{r}=L(\vec{h}) \times B(\vec{h})
$$

Here, $L(\vec{h})$ is nothing else but the reciprocal lattice and $B(\vec{h})$ the structure factor, which one may recognize as a different representation in Eq. (27).

\subsection{Crystal chemistry}

Although the aim of this chapter is the description of diffraction as a method, we should have a brief glance on what we will expect to elucidate, and that is in particular the nuclear or electronic (and eventually magnetic) structure of crystalline solids (i.e., the majority of condensed matter surrounding us). It goes too far to do the "zoology" of crystal structures here, but some general principles of crystalline structures and some important ones, one will find frequently in materials relevant to energy conversion and storage as well as anywhere else, shall be presented here nevertheless.

\subsubsection{Dense packing}

Kepler's conjecture states that no other arrangement of equally sized spheres filling space has a greater average density than the one of face-centred cubic close (ccp or fcc) or hexagonal close packing (hcp). This density is $\pi /(3 \sqrt{2}) \approx 74.05 \%$

In both types of dense packing the atoms (considered to be identical spheres) occupy the points of a Bravais lattice. The fcc packing corresponds to the face-centred cubic Bravais lattice, while the hcp structure corresponds to two interpenetrating simple (primitive) hexagonal Bravais lattices, displaced vertically by a distance $c / 2$ along the common $c$-axis (the stacking direction $\overrightarrow{a_{3}}$ of two-dimensional triangular nets, spanned by two primitive vectors $\overrightarrow{a_{1}}$ and $\overrightarrow{a_{2}}$ of equal length $a=b$, perpendicular to $\overrightarrow{a_{3}}$, and at an angle of $\gamma=60^{\circ}$, which form, by stacking them directly above each other at the distance $c$, the simple hexagonal Bravais lattice).

Each point of a close packing lattice has the same number of nearest neighbours (or touching spheres if one considers atoms as such). This number is a property of the lattice and called coordination number of the lattice. The concept can be extended to arrays of points that are not Bravais lattices, i.e., crystal structures with a basis of more than one atom. The coordination number is 12 , the highest possible 

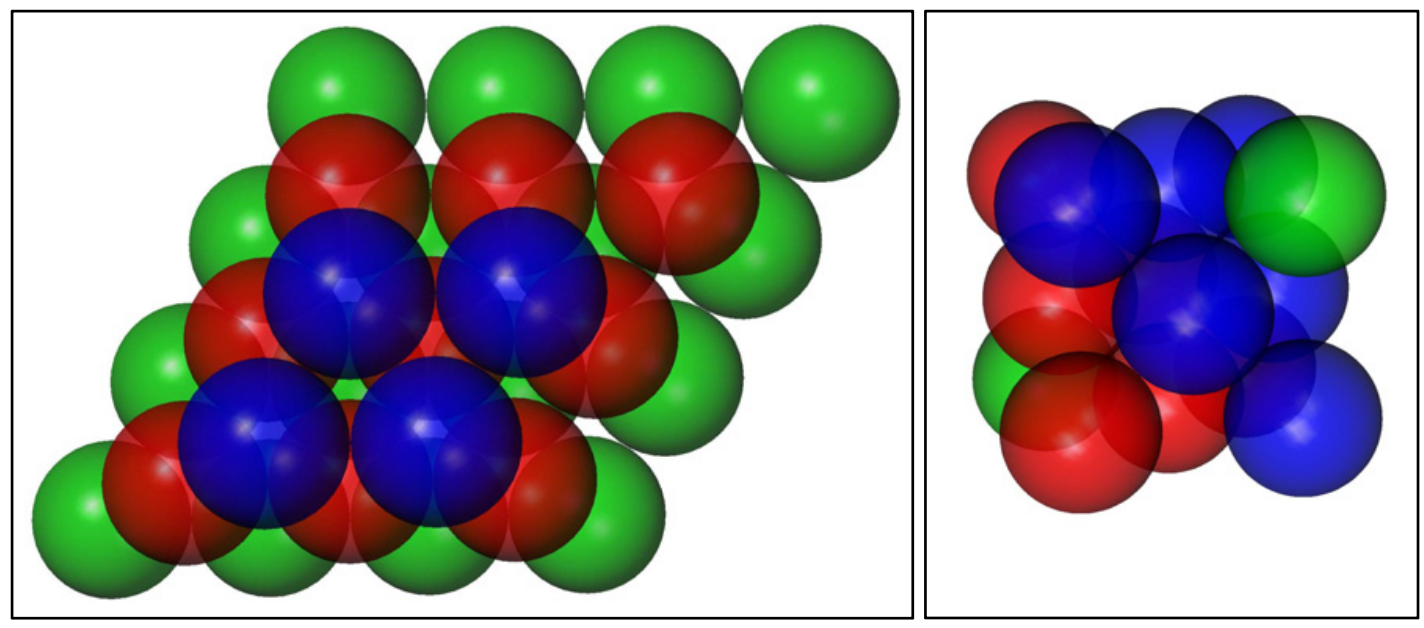

Figure 13. Schematic of cubic close packing. If a layer "A" is represented by green spheres, the red spheres represent a layer "B" and the blue ones "C". In the left panel three layers are viewed in direction of the direction of stacking. In the right one those spheres out of four layers are represented, which form a face-centred cube, viewed in the direction of the four-fold axis. Two spheres of the first and the last layer "A", and three of each layer "B" and "C" form the eight corners, three of each layer " $B$ " and " $C$ " form the centres of the six faces.
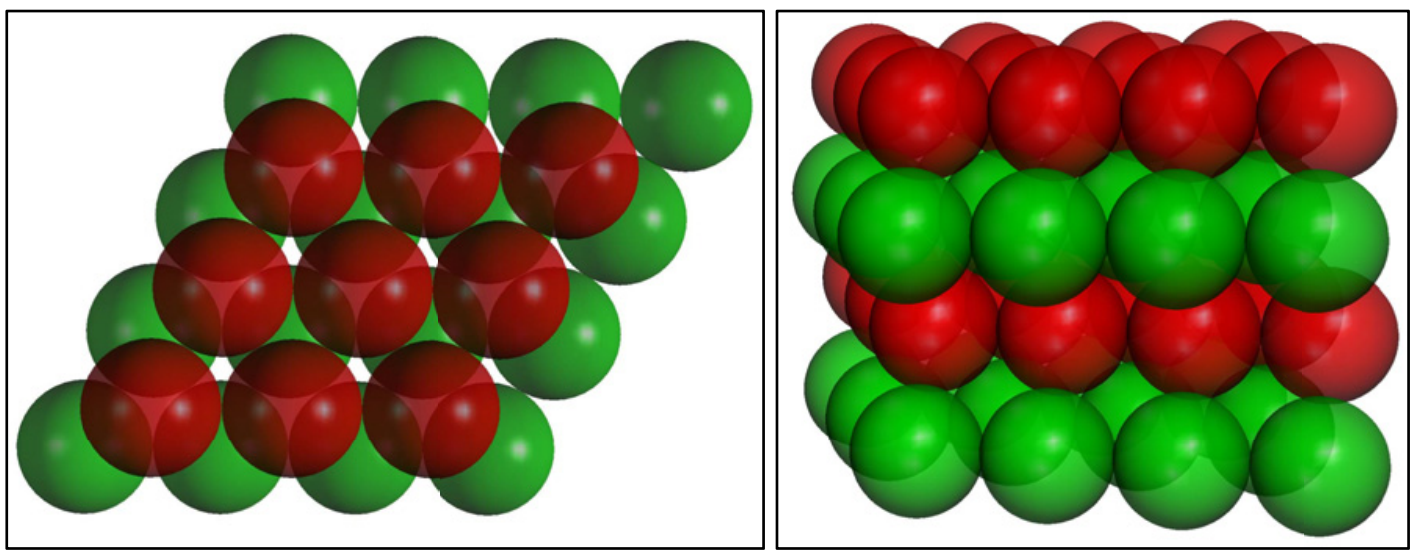

Figure 14. Schematic of hexagonal close packing. If a layer "A" is represented by green spheres, the red spheres represent a layer "B". In the left panel two layers are viewed in direction of the $c$ axis, in the right one four layers viewed perpendicular to this direction.

one for a Bravais lattice and for a packing of identical spheres, for both types of close packing. Due to generally attractive forces between atoms, i.e., identical ones, one would expect dense packed structures being the favoured arrangement of most monoatomic solids with isotropic efficiently attractive forces between atoms. And indeed, most metals and noble gases form, either a fcc (most noble gases, many transition metals, most noble metals) or a hop structure (He, light alkaline earths, most rare earths, $\mathrm{Zn}$-group). Some of them form both (Ce, Sc, Co, La, Pt).

One should mention here, that all kinds of "interbreeds" of hexagonal and cubic close packing are possible. The fcc packing corresponds to the stacking of the same aforementioned two-dimensional triangular nets, identical layers in three different translational states (let us call them A, B and C),

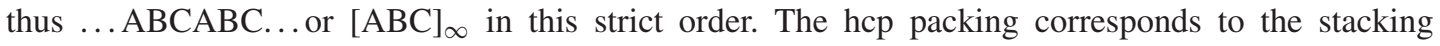



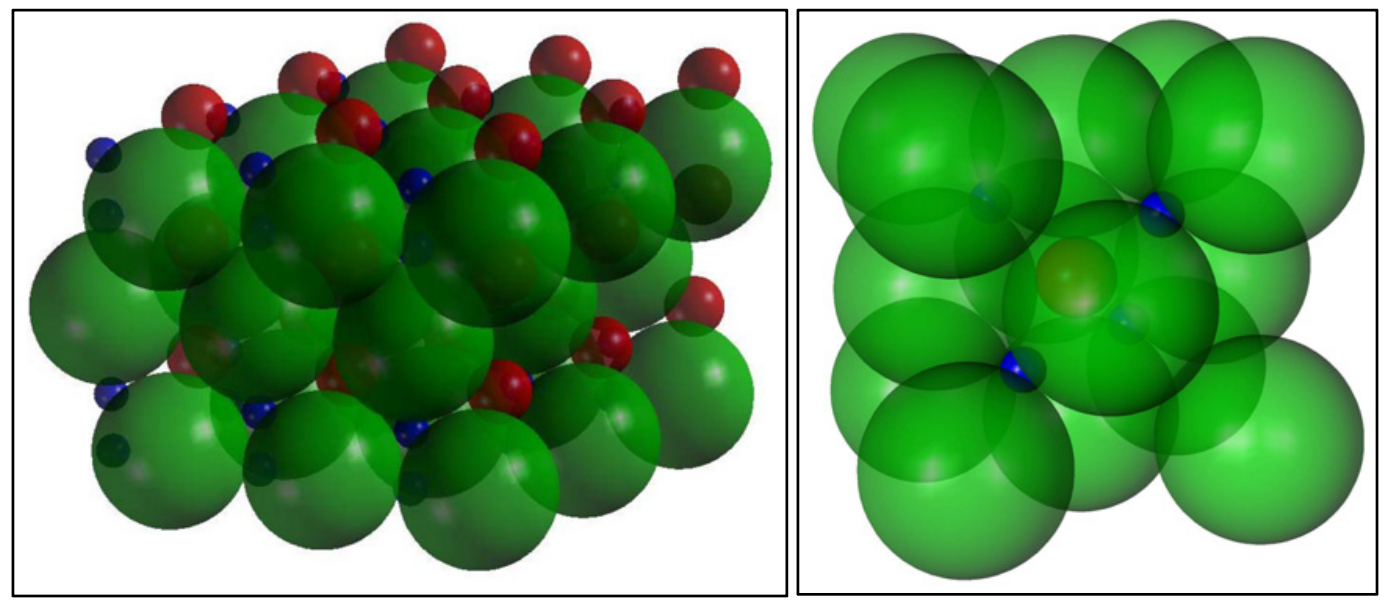

Figure 15. Hexagonal (left) and cubic (right) close packing of equal spheres (anions, green) with octahedral (red) and tetrahedral (blue) holes filled with largest possible fitting spheres (cations).

$\ldots \mathrm{ABAB} \ldots$ or $[\mathrm{AB}]_{\infty}$. In other words, in fcc stacking every layer is different from the two preceding ones and every third layer is identical. In hep stacking every second layer is identical, and a layer is only different from the directly preceding ones. These "stacking rules" are not necessarily strictly followed throughout the crystal. One can either observe different kinds of two-dimensional "stacking faults" of relatively low frequency, where eventually a hexagonal stacking rule is found from time to time (nearly) randomly in a cubic stacking or vice versa, or one can have an repeated specific sequence of hexagonal and cubic rules, with the long-range order of a crystal is maintained and such ordered variants are called "polytypes". One example is samarium, which forms a polytype with alternately two hexagonal (h) and one cubic (c) stacking rules (hhc), resulting in a layer sequence $[\mathrm{ABABCBCAC}]_{\infty}$, whereas other rare earth metals take a structure with simple alternate stacking $(\mathrm{hc})$, resulting in a layer sequence $[\mathrm{ABAC}]_{\infty}$.

Another view on close packing is that of stacking cannonballs (being the first problem documented, where one was seeking the best possible pacing of equal spheres). If one starts from a trigonal twodimensional layer $\mathrm{A}$ as described above, the next layer B, equally trigonal, will have its balls all above alternate interstices (formed by three balls below) of the first layer A. Only every second interstice can be occupied, and the second layer thus forms a trigonal layer identical to the first one, just translated. There are exactly two ways of translating the layer A to become a layer B, as they are perfectly equivalent at this point, we shall call them B anyway. The third layer now will have its balls in the interstices of the second layer, and here again, we have to ways of translating the original layer. Either we have again the original layer $\mathrm{A}$, in which case we have a hexagonal symmetry and we are on our way to a hexagonal dense packing with alternate layers $\mathrm{A}$ and $\mathrm{B}$, or we use the remaining translation to have a layer $\mathrm{C}$, different (in translation) from the two previous ones, and we break the hexagonal symmetry and we are on our way to a cubic dense packing.

Although the two ways of packing look so similar, with always the same coordination number, there are significant differences. First, the hexagonal packing has more degrees of freedom, the lattice parameter in the stacking direction has an ideal value of $c=\sqrt{ }(8 / 3) \cdot a$, corresponding to perfect close packing, from which many elements deviate, see Table 3. Second, as already mentioned, both ways of stacking leave every second interstice above a layer unoccupied. The centres of these empty interstices lay exactly between the two layers, and are surrounded by six balls (coordination number $=6$ ), three below and three above, at same distance, forming an octahedron. There is one octahedron gap for each sphere constituting the packing, and one can easily find, that the maximum radius of a sphere fitting in 
Table 3. Elements crystalling in cubic (left) and hexagonal (right) close-packed structures.

$\begin{array}{lcclcccc}\text { Element } & \mathrm{a} / \AA & \mathrm{V}_{\text {atom }} / \AA^{3} & \mathrm{Element} & \mathrm{a} / \AA & \mathrm{c} / \AA & \mathrm{c} / \mathrm{a} & \mathrm{V}_{\text {atom }} / \AA^{3} \\ \mathrm{Ni} & 3.52 & 10.9 & \mathrm{Be} & 2.29 & 3.58 & 1.56 & 8.13 \\ \beta \text {-Co } & 3.55 & 11.2 & \alpha-\mathrm{Co} & 2.51 & 4.07 & 1.62 & 11.10 \\ \mathrm{Cu} & 3.61 & 11.8 & \mathrm{Ru} & 2.70 & 4.28 & 1.59 & 13.51 \\ \mathrm{Rh} & 3.80 & 13.7 & \mathrm{Os} & 2.74 & 4.32 & 1.58 & 14.04 \\ \mathrm{Ir} & 3.84 & 14.2 & \mathrm{Re} & 2.76 & 4.46 & 1.62 & 14.71 \\ \mathrm{Pd} & 3.89 & 14.7 & \mathrm{Zn} & 2.66 & 4.95 & 1.86 & 15.17 \\ \mathrm{Pt} & 3.92 & 15.1 & \mathrm{Ti} & 2.95 & 4.69 & 1.59 & 17.67 \\ \mathrm{Al} & 4.05 & 1.6 & \mathrm{Cd} & 2.98 & 5.62 & 1.89 & 21.61 \\ \mathrm{Au} & 4.08 & 17.0 & \mathrm{Hf} & 3.20 & 5.06 & 1.58 & 22.44 \\ \mathrm{Ag} & 4.09 & 17.1 & \mathrm{Mg} & 3.21 & 5.21 & 1.62 & 23.25 \\ \mathrm{Ne} @ 4.2 \mathrm{~K} & 4.43 & 21.7 & \mathrm{Zr} & 3.23 & 5.15 & 1.59 & 23.27 \\ \mathrm{Sc} & 4.54 & 23.4 & \mathrm{Sc} & 3.31 & 5.27 & 1.59 & 25.00 \\ \delta \text {-Pu } & 4.64 & 25.0 & \mathrm{Tl} & 3.46 & 5.53 & 1.60 & 28.67 \\ \mathrm{~Pb} & 4.95 & 30.3 & \mathrm{Lu} & 3.50 & 5.55 & 1.59 & 29.44 \\ \mathrm{Th} & 5.08 & 32.8 & \mathrm{Tm} & 3.54 & 5.55 & 1.57 & 30.12 \\ \mathrm{Ce} & 5.16 & 34.3 & \mathrm{Er} & 3.56 & 5.59 & 1.57 & 30.68 \\ \mathrm{Pr} & 5.16 & 34.3 & \mathrm{Ho} & 3.58 & 5.62 & 1.57 & 31.19 \\ \mathrm{Ar} @ 4.2 \mathrm{~K} & 5.26 & 36.4 & \mathrm{Dy} & 3.59 & 5.65 & 1.57 & 31.53 \\ \mathrm{La} & 5.30 & 37.2 & \mathrm{~Tb} & 3.60 & 5.69 & 1.58 & 31.93 \\ \mathrm{Yb} & 5.49 & 41.4 & \mathrm{He} @ 2 \mathrm{~K} & 3.57 & 5.83 & 1.63 & 32.17 \\ \mathrm{Ca} & 5.58 & 43.4 & \mathrm{Y} & 3.65 & 5.73 & 1.57 & 33.06 \\ \mathrm{Kr} @ 58 \mathrm{~K} & 5.72 & 46.8 & \mathrm{Gd} & 3.64 & 5.78 & 1.59 & 33.16 \\ \mathrm{Sr} & 6.08 & 56.2 & \mathrm{Nd} & 3.66 & 5.90 & 1.61 & 34.22 \\ \mathrm{Xe} @ 58 \mathrm{~K} & 6.20 & 59.6 & \mathrm{Ce} & 3.65 & 5.96 & 1.63 & 34.83 \\ & & & \mathrm{Pt} & 3.67 & 5.92 & 1.61 & 34.53 \\ & & & \mathrm{La} & 3.75 & 6.07 & 1.62 & 36.96 \\ & & & \mathrm{ideal} \text { ratio } & \mathrm{c} / \mathrm{a}= & 2 \sqrt{ }(2 / 3) & 1.63 & \\ & & & & & & & \end{array}$

this gap is $(\sqrt{ } 2-1) \approx 41 \%$ of the radius of the close-packed spheres. Smaller gaps are enclosed by three spheres of the lower layer forming an interstice and a sphere of the next layer, lying on this interstice. Here, we have a centre not exactly between the two layers, coordinated by a tetrahedron formed by the close packed spheres. There are two tetrahedral gaps for each sphere constituting the packing. The maximum radius of a sphere fitting in this gap is $(\sqrt{ } 6-2) / 2 \approx 22 \%$ of the radius of the closepacked spheres. Numbers and sizes of these octahedral and tetrahedral gaps are the same for both types of packing, but in the hexagonal case, the octahedral ones $(\mathrm{O})$ share common trigonal faces so to form linear channels through the structure in stacking direction $c$. The tetrahedral gaps in both packing types have two orientations ( $\mathrm{T}_{+}$and $\mathrm{T}_{-}$), but in the hexagonal case, pairs of two opposite oriented tetrahedrons share a common face, whereas in the cubic case $\mathrm{T}_{+}$and $\mathrm{T}_{-}$share common edges, whilst $\mathrm{T}_{+}$share corners among each other, so do $\mathrm{T}_{-}$.

Now, why focussing so much on these gaps? This is, because many, if not most inorganic crystal structures of some ionic bonding character, as well as alloys, can be derived from some dense packing structure. The bigger spheres, typically the anions, form an hcp or fcc structure, and the smaller spheres, usually the cations, fill fully or partially, the octahedral and tetrahedral gaps, depending on the ratio of the size of the spheres (providing one regards atoms and ions as spheres). 2.4.3 lists a few of these structure types.

\subsubsection{Further structure types of elements and metals}

$70 \%$ of the elements fall into four crystal structures: the cubic and the hexagonal close packing, the slightly less compact body centred cubic packing (bcc) or the by comparison loose diamond structure. Beside the above presented close packing structures one observes relatively frequently the 
Table 4. Ionic structures derived from dense close packing of equal spheres (i.e., anions) by filling the tetrahedral $\left(\mathrm{T}_{-}\right.$and $\left.\mathrm{T}_{+}\right)$and octahedral $(\mathrm{O})$ interstices with smaller ions (i.e., cations).

\begin{tabular}{|c|c|c|c|c|c|c|}
\hline formula & cubic (fcc) & hexagonal (hcp) & $\mathrm{T}_{-}$ & $\mathrm{T}_{+}$ & $\mathrm{O}$ & \\
\hline $\mathrm{AX}_{4}$ & $\mathrm{CaTiO}_{3}$ & & - & - & $1 / 4$ & Network of corner-sharing octahedrons \\
\hline $\mathrm{AX}_{3}$ & $\mathrm{CrCl}_{3}$ & $\mathrm{BiI}_{3}$ & - & - & $1 / 3$ & Layers of edge-sharing octahedrons \\
\hline $\mathrm{AX}_{2}$ & $\mathrm{CdCl}$ & $\mathrm{CdI}_{2}$ & - & - & $1 / 2$ & Layers of corner-sharing octahedrons \\
\hline $\mathrm{AX}_{2}$ & $\mathrm{TiO}_{2}$ anatase & $\mathrm{TiO}_{2}$ rutile & - & - & $1 / 2$ & $\begin{array}{l}\text { Tetragonal distorted corner- (hcp) or edge-sharing } \\
\text { (fcc) network of edge-sharing octahedron chains }\end{array}$ \\
\hline $\mathrm{A}_{2} \mathrm{X}_{3}$ & - & $\mathrm{Al}_{2} \mathrm{O}_{3}$ & - & - & $2 / 3$ & $\begin{array}{l}\text { Network of corner-sharing double-octahedrons with } \\
\text { common face }\end{array}$ \\
\hline $\mathrm{AX}$ & $\mathrm{NaCl}$ & NiAs & - & - & 1 & $\begin{array}{l}\text { Edge-sharing network of octahedrons (fcc) or chains } \\
\text { of face-sharing octahedrons (hcp) }\end{array}$ \\
\hline $\mathrm{A}_{3} \mathrm{X}$ & $\mathrm{Li}_{3} \mathrm{Bi}$ & - & 1 & 1 & 1 & $\begin{array}{l}\text { Edge-sharing tetrahedrons share faces with octahe- } \\
\text { drons of edge-sharing network }\end{array}$ \\
\hline $\mathrm{A}_{3} \mathrm{X}_{4}$ & $\mathrm{MgAl}_{2} \mathrm{O}_{4}$ spinel & $\mathrm{Mg}_{2} \mathrm{SiO}_{4}$ olivine & $1 / 8$ & $1 / 8$ & $1 / 2$ & $\begin{array}{l}\text { Edge-sharing octahedron network with isolated } \\
\text { tetrahedrons }\end{array}$ \\
\hline $\mathrm{AX}$ & - & $\gamma-\mathrm{Li}_{3} \mathrm{PO}_{4}$ & $1 / 2$ & $1 / 2$ & - & Edge- and corner sharing tetrahedron network \\
\hline $\mathrm{AX}_{2}$ & $\mathrm{~K}_{2} \mathrm{O}$ antifluorite & - & 1 & 1 & - & Edge-sharing tetrahedron network \\
\hline $\mathrm{AX}$ & $\begin{array}{l}\text { ZnS sphalerite, } \\
\mathrm{CuFeS}_{2}\end{array}$ & $\begin{array}{l}\mathrm{ZnS} \text { wurzite, } \\
\beta-\mathrm{Li}_{3} \mathrm{PO}_{4}\end{array}$ & - & 1 & - & Corner-sharing tetrahedron network \\
\hline
\end{tabular}

body-centred cubic structure, again, with a monoatomic basis, the atoms corresponding to lattice points. The coordination number is slightly lower (8), the neighbouring atoms form a cube. The packing density of equally sized close packed spheres would be $68 \%$ as compared to $74 \%$ in closest packing.

Then, there is the rather loose diamond structure, in which several non-metals (carbon, silicon, germanium, tin) crystallise, elements, which form covalent bonds to neighbours. This structure type allows for the low coordination number of 4 (tetrahedral coordination), resulting in a lower density. But still, this structure can be derived from cubic dense packing, fcc, in two ways: either two fcc lattices interpenetrating each other, with the origin of the second lattice with respect to the first one at $\left(1 / 4^{1 / 4} 4^{1 / 4}\right)$. Or one takes an fcc lattice and fills every second tetrahedral gap with an atom. This is corresponds effectively to the sphalerite structure ( $\mathrm{ZnS}$, see Sect. 2.4.3) with the difference that the later fits much smaller cations in the tetrahedral gaps of the ccp lattice of anions. Consisting of spheres of equal size, these must be $\sqrt{ }(8 / 3)$ smaller in radius than those in an fcc lattice of close packed spheres, taking $16 \sqrt{ }(6 / 9)$ times less volume. As a result, the packing density of the diamond structure is about $34 \%$ as compared to the $74 \%$ of the dense pacing.

\subsection{3 $A X, A X_{2}$ and other structure types derived from close packing}

As insinuated before, when leaving the domain of equally sized atoms, the reasonable model for pure elements and many alloys, when moving towards compounds of ionic or covalent character, one will still encounter the conception of close packed lattices, in particular for compounds with an ionic character, such as metal oxides and salts.

As already mentioned in the discussion of the diamond structure, one can fill half of the tetrahedral interstices of the cubic close packing in order to derive the sphalerite (or zincblende) structure, the cubic modification of $\mathrm{ZnS}$, where big sulphide anions form the close-packed lattice and the Zn-cations occupy the holes. If one starts from the hexagonal close packing of sulphide instead, filling half of its tetrahedral interstices, one derives similarly the other modification of $\mathrm{ZnS}$, the wurzite structure.

Filling half of the tetrahedral interstices is possible for both, cubic and hexagonal close packing (the coordinating tetrahedral around the cations are connected by corners), not so for filling all tetrahedral interstices. This is the case for the so-called anti-fluorite structure formed, e.g., by $\mathrm{K}_{2} \mathrm{O}$, where oxygen 
forms cubic close packing and potassium occupies all tetrahedral interstices, which are connected by common edges. In the hexagonal analogue, these tetrahedral would be connected pair-wise by a common face, which might still be possible for two octahedrons (structures with face-sharing coordination octahedrons do exist), but not for the small tetrahedrons, the central cations (of equal charge) coming too close to each other (closer in fact than the closest distance to the nearest anions) to be stable.

The probably most straightforward AX-structure is the sodium chloride structure, where all octahedral interstices of a cubic close packing of chloride anions are occupied by sodium cations. A geometrical condition is - when considering ions as stiff spheres - that the radius of the small cations is not larger than $(\sqrt{ } 2-1) \approx 0.41$ times the radius of the bigger anions, which are touching each other.

Another equally straightforward structure is the caesium chloride structure, which - however - is not derived the same way from close packing of anions, but from a simple cubic Bravais lattice with a basis of an anion at the origin (i.e., anions occupy all corners of a cubic unit cell). The cation is at the cube centre, surrounded by eight equally distant anions, a cubic coordination. Considering the anions as stiff spheres touching each other, the cation radius must be smaller than $(\sqrt{ } 3-1) \approx 0.73$ times the anion radius, i.e., this structure type is preferred, as compared to the $\mathrm{NaCl}$ (halite) structure, for bigger cations. One can describe the $\mathrm{CsCl}$ structure as well as a bodycentred Bravais lattice where the atoms at the cube centres (i.e., cations) are different from those at the corners (i.e., anions). This latter description, however, is misleading, as one would observe such a body centred Bravais lattice in a diffraction experiment only if the scattering from both atoms is exactly identical.

In the sphalerite structure, the cations occupy tetrahedral interstices in a close packing of touching anions and therefore have to be smaller than $(\sqrt{ }(3 / 2)-1) \approx 0.22$ times the radius of the anions, i.e., this structure type is preferred, as compared to the $\mathrm{NaCl}$ structure, for even smaller cations (or correspondingly bigger anions). For further insight into inorganic crystal chemistry the reader may consult textbooks of solid state chemistry, such as [13].

\section{Neutron diffraction}

The focus here shall be on the diffraction of (thermal) neutrons. For the study of atomic-scale structures, X-ray diffraction is the technique of choice, with sources more abundant (an X-ray generator being standard equipment in most laboratories), and with synchrotron sources more brilliant than any conceivable neutron source. Therefore, a typical diffraction experiment will be performed with X-rays and only exceptionally, when X-rays do not deliver the information wanted, neutrons will be used eventually.

Neutrons are nuclear particles, bosons, with a mass close to that of the proton of $m_{\mathrm{n}} \approx 1.675 \cdot 10^{-27} \mathrm{~kg}$. The free neutron decays with a half live time of $\tau \approx 886 \mathrm{~s}$, which is far more than the time from the production of a neutron in a fission or spallation event until a scattering event and its detection: traveling at a speed of about $3 \mathrm{~km} \cdot \mathrm{s}^{1}$ (for thermal neutrons) even in the longest instrument set-ups (a few $100 \mathrm{~m}$ at most for time-of-flight techniques at spallation sources) the neutron's life ends in the detector after a ten-thousandth of its half-life time. The neutron is electrically neutral but possesses a magnetic moment $\mu=\gamma \cdot \mu_{\mathrm{N}}$ with the neutron magnetogyric ratio $\gamma \approx-1.913$ and the nuclear magneton $\mu_{\mathrm{N}}=e \cdot \hbar / m_{\mathrm{p}}$. It is coupled antiparallel to its spin of $s=1 / 2$. The neutron interacts with nuclei through the strong nuclear force and with magnetic moments through the electromagnetic force.

The thermal, thus moving, neutron's wavelength is related to its velocity through the de Broglie relation (57), which governs the neutron's particle-wave dualism:

$$
\lambda=\frac{2 \pi \cdot \hbar}{m_{n} v}
$$


With the neutron's wave vector $\vec{k}$ of length $k$ (the neutron wave number $k=2 \pi / \lambda$ ) and with the same direction as the velocity $v$ we get Eq. (58):

$$
\vec{k}=\frac{m_{n} \vec{v}}{\hbar}
$$

Finally, we can consider the neutron as non-relativistic and get its kinetic energy as $E=\hbar^{2} \cdot k^{2} /\left(2 m_{\mathrm{n}}\right)$.

Now, aware of the scarceness of neutrons, we need to know valid reasons for employing neutron diffraction rather than other probes:

1. Thermal neutrons have a wavelength corresponding to inter-atomic distances and energy similar to elementary excitations in condensed matter.

2. The neutron scattering cross section varies randomly from element to element and from isotope to isotope even of the same element. Therefore neutrons are well adapted to study most light isotopes, i.e., hydrogen. Also, isotope substitution allows contrast variation experiments.

3. The interaction of neutrons with matter is weak; therefore neutrons probe the bulk of samples, not only the surface. Also, quantitative comparisons between neutron scattering data and theoretical models are possible with higher order effects being small and correctable or negligible.

4. Neutrons penetrate matter easily, which allows for neutron scattering experiments on samples in all sorts of sample environment: cryostats, magnets, furnaces, pressure cells, etc. Even very bulky samples, e.g., of up to $10 \mathrm{~cm}$ thickness, depending on the elemental composition can be studied.

5. Neutrons are scattered by magnetic structures or magnetic field gradients due to their magnetic moment. Unpolarized neutrons probe the periodicity and magnitude of magnetic order, while spin-polarized neutrons reveal the direction of atomic magnetic moments.

We shall in the following neglect this last, very important aspect of neutrons, which accounts for about half of all neutron scattering experiments, and concentrate only on the nuclear interaction of neutrons with matter. Whereas the magnetic interaction shows in a certain aspect similarities to X-ray scattering, the nuclear scattering shows some interesting particularities as discussed further in the following sections. As the main responsible for a magnetic moment are the electrons (the magnetic moment of the nucleus is negligible and its weakness needs sub-Kelvin temperatures to show ordering), we will see a form factor in the expression of the structure factor, which rapidly approaches zero for increasing $Q$, as for X-ray diffraction. The crystallographic treatment of magnetic ordering is complicated by the fact, that we do not deal anymore with the simple arrangement of points, i.e., atoms, in space, but with the arrangement of vectors, i.e., magnetic moments.

\subsection{Scattering length}

When we introduced scattering cross sections earlier, we omitted an important aspect of neutron diffraction, the appearance of incoherent scattering, which cannot be grasped with the simple mechanistic approach leading to the (total) scattering cross section. In fact, the total scattering cross section is summed up from the coherent one, which carries the information in a typical diffraction experiment, and the incoherent one, which is often considered (partly wrongly) as a nuisance when it comes to diffraction (elastic scattering) experiments. To understand, it is opportune to derive the socalled scattering length.

This parameter is used for scattering at low energies: when the energy of the scattered particle becomes very small, the (elastic) scattering cross section approaches that of an impenetrable sphere 
whose radius equals the scattering length $b^{20}$ :

$$
\lim _{k \rightarrow 0} \sigma_{\text {elastic }}=4 \pi b^{2}
$$

Neutrons are scattered by the nucleus by the strong nuclear forces, which have a range of a few femtometers $\left(1 \mathrm{fm}=10^{-15} \mathrm{~m}\right)$, five orders of magnitude less than the thermal neutron's wavelength (around $1 \AA=10^{-10} \mathrm{~m}$ ). Therefore, the neutron cannot probe the internal structure of the nucleus, and the scattering from a single nucleus is, by the first-order Born-approximation (see Sect. 3.2), isotropic. An initial neutron can be described as a complex plane wave $\psi_{\mathrm{i}}$ with a normalisation constant $Y$ defined through the density of the initial neutron being $\left|\psi_{i}\right|^{2}=1 / Y$ :

$$
\psi_{i}(\vec{r})=\frac{1}{\sqrt{Y}} e^{i \vec{k}_{\mathrm{i}} \cdot \vec{r}}
$$

From (58) we obtain the particle velocity $v$ of a plane neutron wave as follows:

$$
v=\frac{\hbar k_{f}}{m_{n}}
$$

With this, the corresponding neutron flux becomes

$$
\Psi_{i}=\left|\psi_{i}\right|^{2} v=\frac{1}{Y} \frac{\hbar k_{i}}{m_{n}}
$$

When a neutron is scattered by a single nucleus at a fixed position, the scattered neutron can be described as a spherical wave $\psi_{f}(\vec{r})$ centred at the nucleus position $\vec{r}_{j}$.

$$
\psi_{f}(\vec{r})=\psi_{i}\left(\vec{r}_{j}\right) \frac{-b_{\mathrm{j}}}{\left|\vec{r}-\vec{r}_{j}\right|} e^{i k_{f} \cdot\left|\vec{r}-\vec{r}_{\mathrm{j}}\right|}
$$

Equation (63) is valid only for $\left|\vec{r}-\vec{r}_{j}\right| \gg b_{j}$, which is easily fulfilled with $b_{j}$ in the range of $1 \mathrm{fm}, r$ in the order of $1 \mathrm{~m}$ in a typical experimental setup (i.e., the sample-detector distance), and $r_{j}$ in the order of $1 \mathrm{~mm}$ or less (i.e., the sample size). The negative sign on $b_{j}$ is a convention, through which most nuclei will have a positive value of the scattering length $b_{j}$. Strongly absorbing nuclei will have an imaginary contribution to the scattering length depending on $k$, thus, the neutron's wavelength. With $|\vec{r}| \gg\left|\vec{r}_{j}\right|$, the density of outgoing neutrons can be approached with $\left|\psi_{f}\right|^{2} \approx \frac{b_{j}{ }^{2}}{Y r^{2}}$. With this, the number of neutrons intersecting a small surface $d A$ in a certain time becomes $d A v\left|\psi_{f}\right|^{2} \approx \frac{d A b_{j}{ }^{2}}{Y r^{2}}$. With (61) and a solid angle segment $d \Omega=d A / r^{2}$ (Fig. 3), we obtain the number of neutrons per time in a solid angle segment $d \Omega$ as $\frac{1}{Y} \frac{b_{j}{ }^{2} \hbar k_{f}}{m_{n}} d \Omega$. With a fixed nucleus, and the energy conservation of the neutron (in the case of elastic scattering), we have $k_{\mathrm{i}}=k_{\mathrm{f}}$. With (62) and the expression for the differential scattering cross Sect. (3) we obtain

$$
\frac{d \sigma}{d \Omega}=\frac{1}{\Psi} \frac{\text { neutrons per time in a solid angle segment } d \Omega}{d \Omega}=\frac{\frac{1}{Y} \frac{b_{\mathrm{j}}{ }^{2} \hbar k_{f}}{m_{n}}}{\frac{1}{Y} \frac{\hbar k_{\mathrm{i}}}{m_{n}}}=b_{j}{ }^{2}
$$

With this we have the total scattering cross section for a single nucleus as $\sigma=4 \pi b_{j}{ }^{2}$, which corresponds to (59).

As promised before, in footnote 20 , we shall quickly make an attempt to illustrate, why the cross section is four times the section one would expect for a hard sphere with a radius of the scattering

\footnotetext{
20 The careful reader will notice, that the cross section is four times larger than geometrically the section of a hard sphere with the scattering length $b$ as radius would be. This is, because we have to leave the classical description and go for a quantum mechanical one of soft repulsive spheres or potential wells. We come back to this at the end of this section.
} 
length. Let us consider two cases: First, let the scattering happen at a repulsive soft sphere, which can be described with an always positive potential of $V(r)=V_{0}$ for $r<\mathrm{r}_{0}$, otherwise $V(r)=0$. Without proof, the solution of Schrödinger's equation for this case delivers $b=r_{0} \cdot\left[1+\tanh \left(k_{0} r_{0}\right) /\left(k_{0} r_{0}\right)\right]$, and as the result of the hyperbolic tangent function is always smaller than $1, b$ will be always positive and smaller than the range $2 r_{0}$. In the other case, the one of a spherical potential well, thus, an attractive sphere, we have $V(r)=-V_{0}$ for $r<\mathrm{r}_{0}$. Again, we obtain an expression for the scattering length, $b=r_{0} \cdot\left[1+\tan \left(k_{0} r_{0}\right) /\left(k_{0} r_{0}\right)\right]$, with $k_{0}=\sqrt{ }\left(2 m_{\mathrm{n}} V_{0} / \hbar^{2}\right)$. As the tangent function can take values larger than one, the sign of $b$ depend on the details. Further, the $b$ diverges for $k_{0} r_{0}$ approaching $\pi / 2+n \pi$, which corresponds to resonant scattering and the appearance of linked states (neutron capture).

For the elastic neutron scattering experiments we intend to do, we do not need, we do not even want to know the exact shape of the potential $V(r)$. Therefore it is useful to construct an efficient potential that produces the same result on the scattered wave, without any internal structure. The most evident choice is a point.

\subsection{Born approximation}

Let's (try to) keep it short here (for a more thorough treatment of the quantum mechanics of scattering the interested reader may take up one of the classical textbooks, such as the ones from Squires [14] or Marshall and Lovesey [15]: each probing neutron, in absence of a potential, behaves as a free particle describable by a planar wave function. Any potential comes in as a perturbation: each point in space, where the potential is not zero, becomes a potential source of a spherical wave. The amplitude of this wave depends on the strength of the interaction, i.e., the potential, and the wave function at the point of interaction (if the probability density at this point is zero, no spherical wave will be created, regardless the potential). All the mathematical difficulty resides in the fact, that the wave function is at once the origin and the result of the perturbation. One can tackle this relatively easily by using an integral expression, which allows for iteration, rather than a differential expression. At some point the so-called Green-operator $\mathbf{G}_{0}$ is introduced (65).

$$
\mathbf{G}_{0}\left|\psi_{\vec{k}}\right\rangle=\int d^{3} r^{\prime} G_{0}\left(\vec{r}, \vec{r}^{\prime}\right) \psi_{\vec{k}}\left(\vec{r}^{\prime}\right)
$$

Here we use the Green function $G_{0}(66)$ that solves the Schrödinger equation for a punctual source, which shall be given here without deduction and just for information:

$$
G_{0}\left(\vec{r}, \vec{r}^{\prime}\right)=-\frac{1}{4 \pi} \cdot \frac{2 m_{n}}{\hbar^{2}} \cdot \frac{e^{i k \cdot \vec{r}-\vec{r}^{\prime} \mid}}{\left|\vec{r}-\vec{r}^{\prime}\right|}
$$

With this, we obtain the so-called Lippmann-Schwinger Eq. (67), of crucial importance in any perturbation computation.

$$
\left|\psi_{\vec{k}}\right\rangle=\left|\psi_{\vec{k}}^{0}\right\rangle+\mathbf{G}_{0} V\left|\psi_{\vec{k}}\right\rangle=\left|\psi_{\vec{k}}^{0}\right\rangle+\mathbf{G}_{0} V\left|\psi_{\vec{k}}^{0}\right\rangle+\mathbf{G}_{0} V \mathbf{G}_{0} V\left|\psi_{\vec{k}}\right\rangle=\cdots
$$

Pushing this iteration to infinity leads finally to the so-called Born-series (68).

$$
\left|\psi_{\vec{k}}\right\rangle=\sum_{n=0}^{\infty}\left(\mathbf{G}_{0} V\right)^{2}\left|\psi_{\vec{k}}^{0}\right\rangle
$$


In this series, a wave operator $\Omega(69)$ produces the scattered wave function out of the wave function of an unperturbed wave.

$$
\Omega=\sum_{n=0}^{\infty}\left(\mathbf{G}_{0} V\right)^{n}
$$

The infinite Born-series may be truncated at a certain order depending on the interaction between probe (neutron) and sample. For the relatively slow thermal neutrons, this interaction turns out to be sufficiently weak, permitting to presume that the wave function is sufficiently unperturbed to use the expression for an unperturbed wave as source. Thus, in this so-called Born approximation ${ }^{21}$, we apply the perturbation method to the scattering by an extended sample. The incident wave function, rather than the total wave function is considered as the relevant wave function at each point in the scattering sample. In other words, we presume that all atoms of a sample see the neutrons arriving as if no other atoms were present. With the number of diffused neutrons sufficiently low to neglect any attenuation of incident flux ${ }^{22}$ we can ourselves limit to the lowest order of the Born-series, the first-order Born-approximation (70):

$$
\left|\psi_{\vec{k}}\right\rangle=\left|\psi_{\vec{k}}^{0}\right\rangle+\mathbf{G}_{0} V\left|\psi_{\vec{k}}^{0}\right\rangle
$$

With this, (65), (66) and (70), we have the following expression for the wave function:

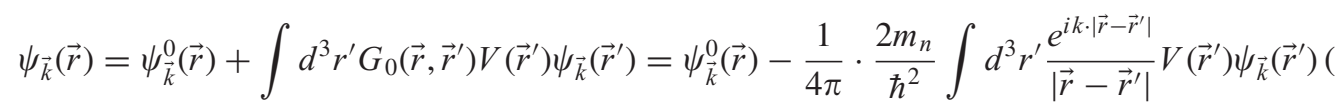

For positions $\vec{r}$ far from the sample, thus, for large values of $|\vec{r}|$ as compared to the range $\left|\vec{r}^{\prime}\right|$ of the potential (in optics, this would be the Fraunhofer diffraction condition) we can rewrite the distance $\left|\vec{r}-\vec{r}^{\prime}\right|$ as follows (with $\hat{r}$ being the unit vector with the direction of $\vec{r}, \hat{r}=\vec{r} / r$ :

$$
\left|\vec{r}-\vec{r}^{\prime}\right|=\sqrt{\left(\vec{r}-\vec{r}^{\prime}\right)^{2}}=\sqrt{r^{2}+r^{\prime 2}-2 \vec{r} \cdot \vec{r}^{\prime}} \approx r\left(1-\vec{r} \cdot \vec{r}^{\prime} / r^{2}\right)=r-\hat{r} \vec{r}^{\prime}
$$

Thus, in the term $e^{i k \cdot\left|\vec{r}-\vec{r}^{\prime}\right|} /\left|\vec{r}-\vec{r}^{\prime}\right|$ of Eq. (71), the denominator $\left|\vec{r}-\vec{r}^{\prime}\right|$ varies very little with $r^{\prime}$ and can be replaced by $r$, while this is not true for the numerator: the variation of the exponential term varies strongly, provided the distance $r^{\prime}$ is close to the wavelength $\lambda=2 \pi / k$. Therefore the proper choice of wavelength is of high importance. In other words, it comes in very handy, that thermal neutrons have wavelengths close to interatomic distances. With these simplifications, we obtain from (71) the following:

$$
\begin{array}{r}
\lim _{r \rightarrow \infty} \psi_{\vec{k}}(\vec{r})=\psi_{\vec{k}}^{0}(\vec{r})-\frac{1}{4 \pi} \cdot \frac{2 m_{n}}{\hbar^{2}} \cdot \frac{1}{r} \int d^{3} r^{\prime} e^{i k \cdot\left|\vec{r}-\vec{r}^{\prime}\right|} V\left(\vec{r}^{\prime}\right) \psi_{\vec{k}}\left(\vec{r}^{\prime}\right) \\
=\frac{e^{i \vec{k} \cdot \vec{r}}}{\sqrt{8 \pi^{3}}}-\frac{1}{4 \pi} \cdot \frac{2 m_{n}}{\hbar^{2}} \cdot \frac{e^{i k \cdot r}}{r} \int d^{3} r^{\prime} e^{i k \cdot\left|\vec{r}-\vec{r}^{\prime}\right|} V\left(\vec{r}^{\prime}\right) \psi_{\vec{k}}\left(\vec{r}^{\prime}\right)
\end{array}
$$

The scattering amplitude $P_{\vec{k}}(\Omega)$ appears in the general solution of Schrödinger's equation for elastic scattering at a static potential $V(\vec{r})$ rapidly decreasing to zero with increasing distance $\vec{r}$ (with the normalisation volume $\left.Y=8 \pi^{3}\right)$ :

$$
\lim _{r \rightarrow \infty} \psi_{\vec{k}}(\vec{r})=\frac{1}{\sqrt{8 \pi^{3}}}\left(e^{i \vec{k} \vec{r}}+P_{\vec{k}}(\Omega) \frac{e^{i \vec{k} \cdot \vec{r}}}{r}\right)
$$

\footnotetext{
21 The Born-approximation must not be confused with the Born-Oppenheimer approximation, crucial for quantum chemistry since its very beginnings in 1927, which allows the computation of the wave function of an average size molecule by solving the time independent Schrödinger equation through breaking the wave function into its electronic and nuclear components.

22 The Born approximation may not always be applicable despite a low scattering length, e.g., for neutron diffraction on a single crystal of good quality. Strong scattering for wave vectors obeying Bragg's law lead to strong beam attenuation.
} 
Comparing (73) and (74) allows to identify the scattering amplitude as follows:

$$
P_{\vec{k}}(\Omega)=-\frac{1}{2 \pi} \cdot \frac{m_{n}}{\hbar^{2}} \int d^{3} r^{\prime} e^{-i k \hat{r} \cdot \vec{r}^{\prime}} e^{i k \cdot \vec{k} \cdot \vec{r}^{\prime}} V\left(\vec{r}^{\prime}\right)
$$

With the vector definitions $\vec{k}_{\mathrm{i}}:=\vec{k}, \vec{k}_{f}:=k \hat{r}$ and $\vec{Q}:=\vec{k}_{\mathrm{i}}-\vec{k}_{f}$, as well as $\langle r \mid \vec{k}\rangle=\frac{e^{i \vec{k} \cdot \vec{r}}}{\sqrt{8 \pi^{3}}}$ we obtain the following expression, with the definition of a transition operator $\mathbf{T}=\mathbf{V} \Omega$ :

$$
P_{\vec{k}}\left(\vec{k}_{\mathrm{i}}, \vec{k}_{f}\right)=-\frac{4 \pi^{2} m_{n}}{\hbar^{2}}\left\langle\vec{k}_{f}|V| \psi_{\vec{k}}\right\rangle=-\frac{4 \pi^{2} m_{n}}{\hbar^{2}}\left\langle\vec{k}_{f}|V \Omega| \vec{k}_{i}\right\rangle=-\frac{4 \pi^{2} m_{n}}{\hbar^{2}}\left\langle\vec{k}_{f}|\mathbf{T}| \vec{k}_{i}\right\rangle
$$

In the case of the Born-approximation, the wave operator $\Omega$ approaches 1 and the transition operator $\mathbf{T}$ approaches $V$, resulting in the following relation:

$$
P_{\vec{k}}\left(\vec{k}_{i}, \vec{k}_{f}\right)=-\frac{4 \pi^{2} m_{n}}{\hbar^{2}}\left\langle\vec{k}_{f}|V| \vec{k}_{i}\right\rangle
$$

The latter can be written as follows (which results as well directly from (75) by using the vector definitions):

$$
P_{\vec{Q}}(\Omega)=\frac{1}{2 \pi} \cdot \frac{m_{n}}{\hbar^{2}} \int d^{3} r^{\prime} e^{i \vec{Q} \cdot \vec{r}^{\prime}} V\left(\vec{r}^{\prime}\right)
$$

Even if the reader may not want to follow how we arrived here, this result is a remarkable one, as it says nothing else than that the scattering amplitude is proportional to the Fourier transform of the interaction potential, thus the scattering density distribution. Through the Born-approximation we get the tools to link the scattering amplitude to an intrinsic property of the sample.

\subsection{Fermi's golden rule}

In order now to treat a sample with several scatterers, we shall take expression (76) again:

$$
P_{\vec{k}}(\Omega)=-\frac{4 \pi^{2} m_{n}}{\hbar^{2}}\left\langle\vec{k}_{f}|\mathbf{T}| \vec{k}_{i}\right\rangle
$$

In this formulation, the cross section becomes:

$$
\frac{d \sigma}{d \Omega}=-\frac{16 \pi^{4} m_{n}^{2}}{\hbar^{4}}\left|\left\langle\vec{k}_{f}|\mathbf{T}| \vec{k}_{i}\right\rangle\right|^{2}
$$

One can express this equally as follows:

$$
d \sigma_{\vec{k}_{\mathrm{i}} \rightarrow \vec{k}_{f}}=r^{2} \frac{w_{\vec{k}_{\mathrm{i}} \rightarrow \vec{k}_{f}}}{\Psi_{\vec{k}_{\mathrm{i}}}} d \Omega
$$

Here, $w_{\vec{k}_{\mathrm{i}} \rightarrow \vec{k}_{f}}$ designs the probability of a transition from state $\vec{k}_{\mathrm{i}}$ to $\vec{k}_{\mathrm{i}}$ and $\Psi_{\vec{k}_{\mathrm{i}}}$ the initial flux. With the introduction of the density of states $\rho_{\vec{k}}\left(E_{f}\right)=\frac{m_{n} k_{f}}{\hbar^{2}}$ we obtain an expression, known as Fermi's golden rule:

$$
w_{\vec{k}_{\mathrm{i}} \rightarrow \vec{k}_{f}}=\frac{2 \pi}{\hbar}\left|\left\langle\vec{k}_{f}|\mathbf{T}| \vec{k}_{i}\right\rangle\right|^{2} \rho_{\vec{k}}\left(E_{f}\right)
$$

The matrix element $\left|\left\langle\vec{k}_{f}|\mathbf{T}| \vec{k}_{i}\right\rangle\right|^{2}$ gives the probability of the transition from the state $\left|\vec{k}_{i}\right\rangle$ to the state $\left|\vec{k}_{f}\right\rangle$. This transition is induced by the interaction potential $V$. More precisely, Fermi's golden rule gives the rate of change between the neutron in the single incoming state and a continuum of final states. 


\subsection{The master formula}

Now, we want to consider only neutrons scattered into the solid angle $d \Omega$. With the definition of the density of states as in the previous section above, Fermi's golden rule (82) and the normalisation volume $Y=8 \pi^{3}$ as in Sect. 3.2, we obtain the number of neutrons scattered into $d \Omega$ per time unit as follows:

$$
w_{\vec{k}_{\mathrm{i}} \rightarrow \vec{k}_{f}, d \Omega}=\frac{Y k_{f} m_{n}}{8 \pi^{3} \hbar^{2}} d \Omega\left|\left\langle\vec{k}_{f}|\mathbf{T}| \vec{k}_{i}\right\rangle\right|^{2}
$$

With the incoming flux being $\Psi_{\vec{k}_{\mathrm{i}}}=\frac{v}{Y}=\frac{\hbar k_{\mathrm{i}}}{Y m_{n}}$, we get a short form of the master equation for scattering:

$$
\frac{d \sigma}{d \Omega}=\frac{w_{\vec{k}_{\mathrm{i}} \rightarrow \vec{k}_{f}, d \Omega}}{\Psi_{\vec{k}_{\mathrm{i}}} d \Omega}=Y^{2} \frac{k_{f}}{k_{i}}\left(\frac{m_{n}}{2 \pi \hbar^{2}}\right)^{2}\left|\left\langle\vec{k}_{f}|\mathbf{T}| \vec{k}_{i}\right\rangle\right|^{2}
$$

With $k_{\mathrm{i}}=k_{f}$ for elastic scattering, this is exactly the same as we had in (80). The more complete form for the double differential scattering cross section becomes, with the Born-approximation applied, the following expression, where we have to consider as well the (discrete) states of the sample before and after the scattering event, $\left|\lambda_{i}\right\rangle$ and $\left|\lambda_{f}\right\rangle$ with $\left.\left|\lambda_{i}, \vec{k}_{i}\right\rangle=\left|\vec{k}_{i}\right\rangle \otimes|| \lambda_{i}\right\rangle$ and $\left.\left|\lambda_{f}, \vec{k}_{f}\right\rangle=\left|\vec{k}_{f}\right\rangle \otimes \lambda_{f}\right\rangle$. We have to account as well for the statistical weight of initial states, $p\left(\lambda_{i}\right)$.

$$
\frac{d^{2} \sigma}{d \Omega d E_{f}}=Y^{2} \frac{k_{f}}{k_{i}}\left(\frac{m_{n}}{2 \pi \hbar^{2}}\right)^{2} \sum_{\lambda_{i} \lambda_{f}} p\left(\lambda_{i}\right)\left|\left\langle\lambda_{f}, \vec{k}_{f}|V| \lambda_{i}, \vec{k}_{f}\right\rangle\right|^{2} \delta\left(\left(E_{f}-E_{i}\right)-\left(E_{\lambda_{i}}-E_{\lambda_{f}}\right)\right)
$$

However, the casual reader, interested in an occasional diffraction experiment to get structural information, should not despair: this master formula goes above elastic scattering and is at the base of the inelastic scattering experiments, which shall not be further subject of this chapter. It has been mentioned here so some extend for the author's hopeless desire of completeness. However, it will help us as well to understand the occurrence of the neutron-specific phenomenon of incoherent scattering, therefore we have not yet finished with this formula.

\subsection{Form factor and Fermi pseudopotential}

The Fourier transform $V_{j}(\vec{Q})$ of the interaction potential of a single scatterer $j$ in a sample is the form factor of the potential $V_{j}\left(\vec{r}_{j}\right)$ of the scatterer:

$$
V_{j}(\vec{Q})=\int d^{3} r_{j} e^{-i \vec{Q} \cdot \vec{r}_{j}} V_{j}\left(\vec{r}_{j}\right)
$$

With this the matrix term in the master formula (85) becomes factorised as follows:

$$
\left\langle\lambda_{f}, \vec{k}_{f}|V| \lambda_{i}, \vec{k}_{i}\right\rangle=\frac{1}{(2 \pi)^{3}} \sum_{j=1}^{N} V_{j}(\vec{Q})\left\langle\lambda_{f}\left|e^{i \vec{Q} \cdot \vec{R}_{j}}\right| \lambda_{i}\right\rangle
$$

We obtain a constant Fourier transform, if we evaluate the form factor for a potential of very short range, i.e. a point potential, as follows.

$$
V_{j}(\vec{Q})=\frac{2 \pi \hbar^{2}}{m_{n}} b_{j}
$$


Using the following Fermi pseudopotential (a potential that makes the real and the pseudo wavefunctions and potentials match above a certain cutoff radius) we would have got the same result:

$$
V_{e f f}(r) \psi(r)=\frac{2 \pi \hbar^{2}}{m_{n}} b_{j} \delta(\vec{r})
$$

With this, the master formula leads finally back to the simple expression (64) for elastic scattering at a single nucleus. ${ }^{23}$

\subsection{Scattering function}

Without further proof, we obtain with (87) and (88) from the master formula (85) the following expression for the partial differential cross section:

$$
\frac{d^{2} \sigma}{d \Omega d E_{f}}=\frac{k_{f}}{k_{i}} S(\vec{Q}, \omega)
$$

In this compact formulation we have defined a scattering function as follows:

$$
S(\vec{Q}, \omega)=\frac{1}{2 \pi \hbar} \sum_{\lambda_{i}} p\left(\lambda_{i}\right) \sum_{j, j^{\prime}=1}^{N} b_{j} b_{j^{\prime}}^{*} \int_{-\infty}^{\infty} d t\left\langle\lambda_{i}\left|e^{-i \vec{Q} \cdot \vec{R}^{0}} j^{\prime} e^{-i \vec{Q} \cdot \vec{R}_{j}(t)}\right| \lambda_{i}\right\rangle e^{-i \omega t} .
$$

\subsection{Coherent and incoherent scattering}

Let us assume now a general crystalline sample made of atoms of only one element. Even though, the neutron scattering length can vary from nucleus to nucleus, for two reasons, either due to the variation of the nuclear spin-directions relative to the neutron (the scattering length can be very different dependent on whether the nucleus' spin and the one of the scattered neutron is parallel or antiparallel), or, on top of that, due to the presence of several isotopes of the same element, which have different scattering lengths. Isotopes and spin states are randomly distributed in a sample; interactions that could lead to a spin alignment are too weak, and the same applies to isotope distribution. This is something we do not encounter in X-ray diffraction.

Let us define the scattering function as follows:

$$
S(\vec{Q}, \omega)=\sum_{j, j^{\prime}=1}^{N} b_{j} b_{j^{\prime}}^{*} S_{j, j^{\prime}}(\vec{Q}, \omega)
$$

With the following function depending on the chemical state of the sample:

$$
S_{j, j^{\prime}}(\vec{Q}, \omega)=\frac{1}{2 \pi \hbar} \sum_{\lambda_{\mathrm{i}}} p\left(\lambda_{\mathrm{i}}\right) \int_{-\infty}^{\infty} d t\left\langle\lambda_{i}\left|e^{-i \vec{Q} \cdot \vec{R}^{0}{ }_{j^{\prime}}} e^{-i \vec{Q} \cdot \vec{R}_{j}(t)}\right| \lambda_{i}\right\rangle e^{-i \omega t}
$$

In the sum (92) we have to distinguish the distinct members, pairs with $j \neq j^{\prime}$, and the self-terms with $j=j^{\prime}$. The earlier ones are proportional to the squared mean scattering lengths, while the later are proportional to the mean squared scattering lengths. With this, we can split the scattering function in

\footnotetext{
23 One may note here, that the scattering length is sometimes called Fermi length (in particular in French: longueur de Fermi), as it is proportional to the Fermi pseudo-potential.
} 
two parts:

$$
\begin{gathered}
S(\vec{Q}, \omega)=\sum_{j, j^{\prime}=1, j \neq j^{\prime}}^{N} \bar{b}_{j} \overline{b_{j^{\prime}}^{*}} S_{j, j^{\prime}}(\vec{Q}, \omega)+\sum_{j=1}^{N} \overline{b_{j}^{2}} S_{j, j}(\vec{Q}, \omega) \\
=\sum_{j, j^{\prime}=1}^{N} \bar{b}_{j} \overline{b_{j^{\prime}}^{*}} S_{j, j^{\prime}}(\vec{Q}, \omega)+\sum_{j=1}^{N}\left(\overline{b_{j}^{2}}-{\overline{b_{j}}}^{2}\right) S_{j, j}(\vec{Q}, \omega)
\end{gathered}
$$

We can thus define the scattering cross section as a sum of coherent and incoherent cross section:

$$
\begin{gathered}
\sigma_{\mathrm{coh}}=4 \pi(\bar{b})^{2} \\
\sigma_{\mathrm{inc}}=4 \pi\left[\overline{b^{2}}-(\bar{b})^{2}\right]
\end{gathered}
$$

The incoherent scattering is of a quite different nature than the coherent one (we shall skip the full mathematical treatment, which implies the introduction of correlation functions [16]), due to the absence of correlation. As Eq. (94) implies, only interactions of scatterers at one position at a time with (the same) scatterer at the same position (but another time) contribute, whereas any structural information contains the distance between different scattering positions, which does not contribute to the incoherent scattering. Whereas incoherent scattering does not carry structural information, it may well, for the previously reason (correlation of the same atom with itself at different times), carry information about diffusion of atoms. Incoherently scattered neutrons are emitted in all directions without interference. In a typical (elastic) diffraction experiment, incoherent scattering can be regarded as "background" and relatively easily subtracted as such, as it is isotropic, i.e., a "flat" background contribution in the case of powder diffraction. However, it affects counting statistics negatively, among other issues, like multiple scattering effects for strong incoherent scatterers such as hydrogen, which are difficult to correct for. Also, incoherent scattering is easily mistaken for diffuse scattering with which it must not be confused (such as the already mentioned thermal diffuse scattering). In diffraction experiments on liquid and amorphous materials where instead of sharp Bragg peaks broad intensity variations are measured, incoherent scattering is a bigger problem as it needs to be subtracted very carefully over the whole angular range, i.e., $Q$-range, in order not to lead to misinterpretation of the data. One can no longer neglect the inelastic contribution to the incoherent scattering (the Debye-Waller factor gives the ratio of elastic incoherent scattering, which therefore diminishes with increasing wave vector, whereas the inelastic contribution increases respectively) and the wavelength-dependence of neutron detector efficiencies. The so-called Placzek-correction [17, 18] does remedy this but needs a ideally a deeper insight into a material's molecular dynamics, is cumbersome and not free from potential mistakes.

\subsection{Dependence of scattering length on nuclear spin}

Now, there are two origins for an appearance of incoherent scattering. An element may well have several (stable) isotopes occurring naturally at a given proportion ${ }^{24}$. In effect, only a minority of elements - all but one (Ce) with an odd number of protons (Z), i.e., Be, F, Na, Al, P, Sc, V, Mn, Co, As Rb, Y, Nb, $\mathrm{Rh}, \mathrm{In}, \mathrm{I}, \mathrm{Cs}, \mathrm{La}, \mathrm{Pr}, \mathrm{Eu}, \mathrm{Tb}, \mathrm{Ho}, \mathrm{Tm}, \mathrm{Lu}, \mathrm{Ta}, \mathrm{Re}, \mathrm{Au}$ and $\mathrm{Bi}$ - have a single stable isotope. Some of them are accompanied in nature by one $(\mathrm{V}, \mathrm{Rb}, \mathrm{In}, \mathrm{La}, \mathrm{Eu}, \mathrm{Lu}, \mathrm{Ta}$ and $\mathrm{Re})$ or three $(\mathrm{Ce})$ very longliving radioactive isotopes. For In and Re, the radioactive isotope eventually becomes the dominant one.

\footnotetext{
24 One should put a disclaimer here to the term "given": As isotopes are the result of nuclear reactions, e.g., radioactive decay chains, the "natural" distribution of isotopes in an element is likely to change with time, even for stable isotopes. Further, this distribution depends on the place in the Universe, i.e., the precise (stellar) origin of the material, its production event. Even on Earth, elements may have slightly different compositions in different regions, a fact that can be exploited to determine the provenance of materials.
} 
When several isotopes with different scattering lengths are present, incoherent scattering will occur, as isotopes will not chemically order ${ }^{25}$. The mean of the squares of scattering lengths at an element's position in a sample will thus be different from the square of the means of scattering lengths, and a non-zero incoherent cross section arises (95). If an element has only a tiny fraction of other isotopes beside the main one, or, if the scattering lengths are not too different, the incoherent cross section will be small with respect to the coherent one. On the other side, if two isotopes are equally present, and if one has a negative scattering length of a similar amount as the other, positive one, the incoherent cross section becomes overwhelming and the coherent one may even disappear.

But even for an element made from a single isotope, one may observe incoherent scattering. This is due to the nuclear spin $I$ of the isotope. The neutron has a spin of $S=1 / 2$, and we have a combined total spin of $J^{ \pm}=I \pm 1 / 2$ with both values of $J$ resulting in different scattering lengths $b^{ \pm}$. With the quantum mechanical degeneration of the levels of the total spin being $n_{J}=2 J+1$, we obtain $n_{J}^{+}=2 J^{+}+1=2 I+2$ and $n_{J}=2 I$. If the neutron beam is not polarised, all spin states are equally likely and we derive two probabilities for the two spin states with different scattering length.

$$
\begin{aligned}
& \mathrm{p}^{+}=\frac{2 I+2}{4 I+2}=\frac{I+1}{2 I+1} \\
& \mathrm{p}^{-}=\frac{2 I}{4 I+2}=\frac{1}{2 I+1}
\end{aligned}
$$

With this, we obtain the mean and mean square scattering lengths.

$$
\begin{aligned}
\overline{\mathrm{b}} & =\frac{1}{2 I+1}\left((I+1) b^{+}+I b^{-}\right) \\
\overline{\mathrm{b}^{2}} & =\frac{1}{2 I+1}\left((I+1)\left(b^{+}\right)^{2}+I\left(b^{-}\right)^{2}\right)
\end{aligned}
$$

The scattering cross sections are obtained from this, and one finds easily, that there is no incoherent scattering if either the nuclear spin is zero or the two scattering lengths are the same.

$$
\begin{aligned}
\sigma_{\mathrm{coh}} & =4 \pi \frac{\left(I b^{-}+(I+1) b^{+}\right)^{2}}{(2 I+1)^{2}} \\
\sigma_{\mathrm{inc}} & =4 \pi \frac{I(I+1)\left(b^{-}-b^{+}\right)^{2}}{(2 I+1)^{2}}
\end{aligned}
$$

The proton and the neutron both have a spin $I=1 / 2$. In the nucleus, they tend to form spin-cancelling pairs, therefore the nuclear spin is indeed zero for all nuclides with an even number of neutrons $(N)$ and

\footnotetext{
25 It is not exactly true, that isotopes cannot be distinguished chemically. After all, one manages to separate isotopes by different means since nearly 100 years. The different atomic mass $(A)$ has an influence on chemical kinetics, the lighter the element the more the so-called kinetic isotope effect (KIE) becomes significant (as the relative mass change is more important). Quantitatively, the KIE is the ratio of kinetic rate constants of the light and heavy isotopically substituted reactants. It is an essential tool for studying reaction mechanisms. The KIE can be as high as 5 for reactions involving hydrogen (or deuterium) at room temperature, whereas a similar reaction involving carbon is only by $4 \%$ faster with ${ }^{12} \mathrm{C}$ as compared to ${ }^{13} \mathrm{C}$. In the case of hydrogen, on top of that the may be a quantum-mechanical tunneling effect, with an exponential dependency on the isotope mass. The KIE may reach values as high as 25 in some biologically relevant reactions at room temperature, which may illustrate, why at first sight harmless deuterated products are toxic for all life forms. One would need nevertheless to replace a quarter of a human's body's water by heavy water to cause serious damage, thus, accidental or intentional poisoning is very unlikely. (One can nevertheless "produce" fully deuterated bacteria, in order to produce deuterated proteins and other biomolecules. However, many bacteria generations are needed to substitute slowly hydrogen by deuterium in order not to kill the culture.)
} 
an even number of protons $(Z)$, all other nuclides do have a non-zero $\operatorname{spin}^{26}$. Nuclides with an odd mass number $A$ will be fermions, those with an even $A$ bosons, i.e., will have an integer spin. To name a few examples, the proton (hydrogen) has a spin $I=1 / 2$, the deuteron (deuterium) has $I=1$. Among the stable iron nuclides, there is only one, ${ }^{57} \mathrm{Fe}$, which has a non-zero spin of $I=1 / 2$, while the only stable cobalt nuclide, ${ }^{59} \mathrm{Co}$, has $I=7 / 2$. The highest spin of a natural nuclide, with $I=7$, is found in the instable nuclide ${ }^{176} \mathrm{Lu}$, one of the rare naturally abundant bosons, the others are ${ }^{2} \mathrm{H},{ }^{6} \mathrm{Li},{ }^{14} \mathrm{~N}$ and ${ }^{10} \mathrm{~B}$ (stable), as well as ${ }^{40} \mathrm{~K},{ }^{50} \mathrm{~V}$ and ${ }^{138} \mathrm{La}$ (instable). ${ }^{10} \mathrm{~B}$ has the highest spin of a stable boson $(I=3)$. As for fermions, ${ }^{73} \mathrm{Ge},{ }^{83} \mathrm{Kr}{ }^{87} \mathrm{Sr},{ }^{93} \mathrm{Nb},{ }^{113} \mathrm{In},{ }^{115} \mathrm{In}$ (unstable), ${ }^{179} \mathrm{Hf}$ and ${ }^{209} \mathrm{Bi}$, have the highest fermion-spins of all naturally abundant isotopes with $I=9 / 2$.

For $\left(\right.$ bound ${ }^{27}$ ) hydrogen, we measure $b_{+}{ }^{p}=10.82 \mathrm{fm}$ (triplet, spins parallel) and $b_{-}{ }^{p}=-47.4 \mathrm{fm}$ (singlet, spins anti-parallel). The strong negative scattering length of the singlet means that the system is close to a linked state. In the triplet state there is a real linked state, the deuteron, which explains a significant and positive scattering length (see section 0). As, by coincidence, the ratio of the scattering lengths is about the ratio of the probability of the respective quantum states, we obtain with (98) a huge incoherent scattering cross section and a rather small coherent one: $\sigma_{\mathrm{coh}}=1.8$ barn and $\sigma_{\mathrm{inc}}=79.8$ barn.

For deuterium ( $\operatorname{spin} I=1$ ), the situation is quite different. Here, we deal with a quadruplet $\left(b_{+}{ }^{D}=9.5 \mathrm{fm}\right)$ and a duplet $\left(b_{D}^{-}=1.0 \mathrm{fm}\right)$ and obtain $\sigma_{\mathrm{coh}}=5.6 \mathrm{barn}$ and $\sigma_{\text {inc }}=2.0$ barn. This makes it worthwhile to replace hydrogen by deuterium whenever possible for diffraction experiments, as coherent scattering alone carries structural information and any incoherent scattering is only drowning counting statistics in background. On top of that, deuterium shows a negligible absorption, while the one the proton, with $\sigma_{\mathrm{abs}}=0.33$ barn for thermal neutrons, cannot be neglected. The latter is also the reason to favour heavy water over normal water for the moderation of neutrons in a research reactor.

\subsection{Coherence length}

Interference (so, diffraction) is due to the phase shifts of wavelets originating from different places of the scattering sample and having therefore a different optical path from the sample to the point of observation, i.e., the detector. The diffraction images are neat and clear, as long as the scattering of all wavelets is synchronized with well-defined phase relations. However, these conditions are not fulfilled for a non-point source - and especially neutron sources have a significant surface - emitting waves without synchronisation, i.e. not coherently (as a laser would do). Similar applies to the wavelength, the fact that there is not a single wavelength, but a spectrum with a certain bandwidth, which is the case, again, particularly for neutron monochromators, will un-sharpen the interferences.

The longitudinal or temporal coherence length quantifies the latter: For a given frequency $v$ the phase of the complex amplitude of the wave changes by a factor $2 \pi$ over a distance $L=\lambda=c / \nu$. Two waves scattered at the same spot of the sample at the same time and with a frequency difference $\Delta v$ will be totally out of phase after the longitudinal length $L_{c}=c / \Delta v=\lambda^{2} / \Delta \lambda$. This length defines thus the resolution of the diffraction probe in real space.

The effect of not having a point-source leads to with a similar reasoning (difference in optical path must be smaller than the wavelength) to the lateral coherence length $L_{c}=\lambda \cdot L / L_{\mathrm{s}}=\lambda / \theta$ with $L$ being the distance of the sample from the source, $L_{\mathrm{s}}$ the size of the source and $\theta$ the angle intercepting the source as seen from the sample. Again, this coherence length determines the resolution in real space:

\footnotetext{
26 As only elements with an odd number of protons occur eventually in form of a unique isotope, which will thus have a non-zero spin, one will have some potential for incoherent scattering for all elements, either due to a non-zero spin, or due to an isotope distribution, or both.

27 We neglected this little thing so far, as we deal exclusively with bound atoms when using diffraction for crystal structure analysis. We have the following relation between scattering lengths for free and bound atoms: $b_{\text {bound }}=\frac{m_{n}+M}{M} b_{\text {free }}$ with $M$ being the atom's mass. For light atoms the difference is not negligible.
} 


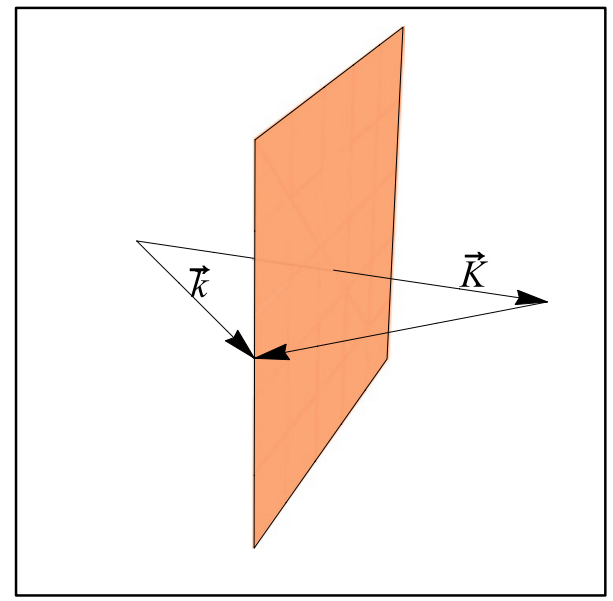

Figure 16. A Bragg plane: with the Laue condition fulfilled, the tip of $\vec{k}$ lies in the plane bisecting $\vec{K}$.

one will not by diffraction determine any distance between two scatterers in a sample, which further than that length apart. A small source (approaching thus the ideal point source), a long distance to the source and a long wavelength contribute positively to this lateral coherence length.

\section{Diffraction instrumentation}

\subsection{The Ewald construction}

For diffraction instruments, some generalities in terms of the geometry are thrown by the Laue condition of the Bragg equation, discussed in 1.4.1 and 1.4.2. In reciprocal space, for a diffraction peak of a crystal to occur, the tip of a wave vector $\vec{k}$ has to lie on one of the Bragg planes, which are perpendicularly bisecting the lines from the origin of the reciprocal space to the reciprocal lattice points $\vec{K}$, as illustrated in Fig. 16. As the Bragg planes represent a discrete family of planes, they do not fill the threedimensional reciprocal space. In general, the tip of an arbitrary wave vector $\vec{k}$ will thus not lie on such a plane. Therefore, for a fixed wavelength and a fixed direction of the incident beam to the crystal orientation, one may well not observe any diffraction peaks at all. To observe experimentally diffraction peaks, one must relax the constraint on $\vec{k}$, either by varying its magnitude, i.e., the incident wavelength, or by varying its direction, i.e., the orientation of the crystal with respect to the incident beam.

The so-called Ewald sphere [19] is a simple geometric construction to visualize the relationships of wave vector, reciprocal lattice and diffraction angle and the different experimental methods. It consists to draw in reciprocal space a sphere with the radius $k$, centred on the tip of the incident wave vector $\vec{k}$ (in consequence, the origin is on the sphere's surface). As shown in Fig. 17, there may be one or more wave vectors $\overrightarrow{k^{\prime}}$ that fulfil the Laue condition, only if there are a reciprocal lattice points on the surface of the sphere. In most cases the construction is simplified to represent two dimensions only in an Ewald circle. Several methods to bring Bragg peaks into observation conditions can be illustrated with this construction.

\subsubsection{The Laue method}

A single crystal of fixed orientation in the incident beam allows for the search and measuring of several Bragg peaks by using a non-monochromatic beam with wavelengths from $\lambda_{1}$ to $\lambda_{0}$. The Ewald sphere 


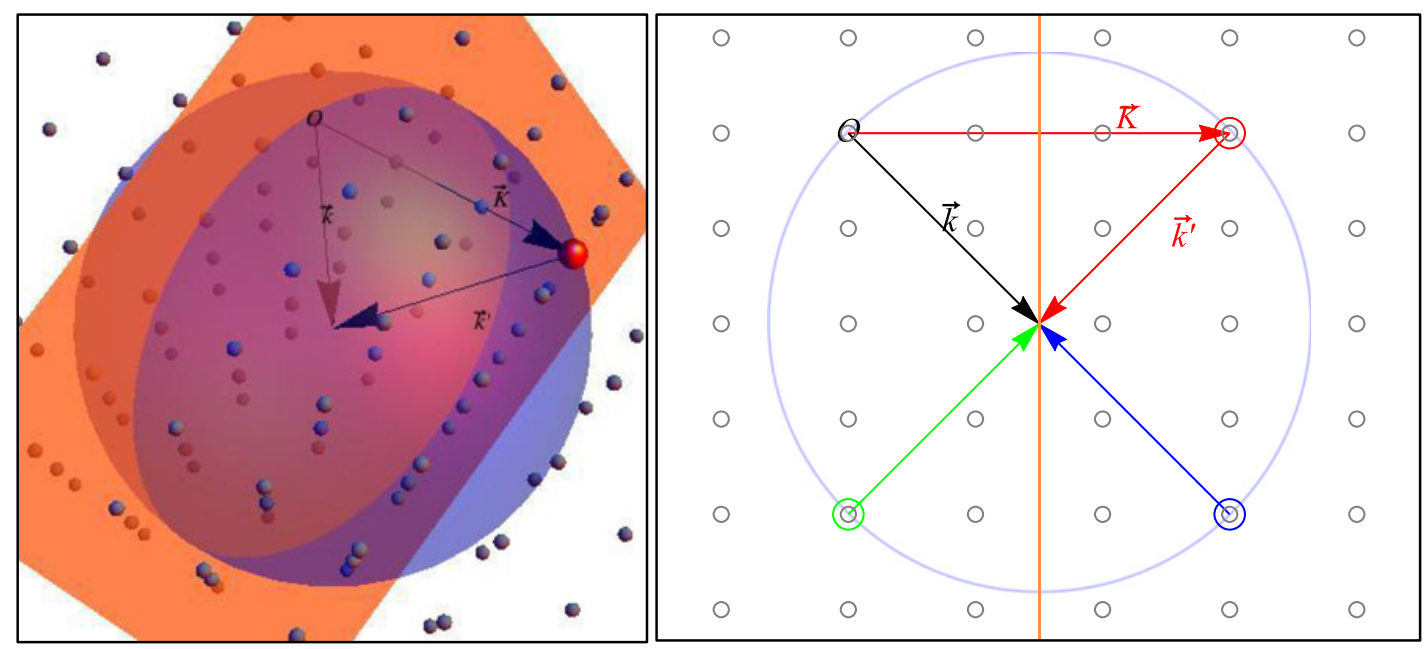

Figure 17. Right: Ewald sphere (blue) with Bragg plane (orange). Lattice points from an orthorhombic primitive Bravais lattice with lattice constants $3 a, 4 \mathrm{a}$ and $2 a$ (grey small spheres), with one lattice vector $(h k l=(300))$ fulfilling the Laue condition (red sphere). The vectors amount to $K=2 \pi 3 / 3 a$ and $k=2 \pi / a \sqrt{2}$, corresponding to $\lambda=a \sqrt{2}$. The diffraction angle in this case is $2 \theta=90^{\circ}$. The Bragg condition is fulfilled: $2 \mathrm{~d} \sin \theta=2 a \sqrt{2} / 2=\lambda$. Left: Ewald circle (blue) for the same situation, covering the $(h k 0)$ lattice vectors (grey). The Bragg plane reduces to a line, and in addition to the reflection (300) one finds that the reflections (340) (blue) and (040) (green) should be possible as well. However, (340) would result in a diffraction angle of $180^{\circ}$, impossible to be observed, whereas (040) gives exactly the same diffraction angle as (300), which means, that the two reflections cannot be distinguished in a powder diffraction experiment (but in a single crystal experiment they will be observed at exactly opposite direction with respect to the crystal orientation).

will then expand into a region between two spheres with correspondingly different radius. Bragg peaks will be observed for all lattice vectors within this region, see Fig. 17. With the wavelength spread one may tune the number of observed Bragg peaks, not too few, not carrying sufficient crystallographic information, but not too many neither, to be still able to resolve individual peaks on the detector and to index them, i.e., attribute reciprocal lattice vectors, thus, Miller indices to them.

\subsubsection{The rotating crystal method}

In this fundamental monochromatic single crystal method (in some sense it is still used on four-circle single crystal neutron diffractometers in order to find first peaks for an initial alignment of the crystal before running through an computer-driven protocol to observe all observable diffraction peaks by orienting the crystal in the beam) the angle of incidence varies by rotating the crystal orientation about a fixed axis in the fixed incident beam. All Bragg peaks observed during the rotation are recorded on a film in the original, now somewhat out-dated X-ray method. The Ewald sphere is fixed in reciprocal space, while the reciprocal lattice rotates about the rotation axis of the crystal. Each lattice point describes a circle around that axis and a Bragg peak appears wherever this circle intersects the Ewald sphere, as shown in Fig. 18.

\subsubsection{The Debye-Scherrer method}

This monochromatic powder diffraction technique is equivalent to the rotation crystal method with the addition that the axis of orientation is varied over all possible orientations. In fact, there is (normally) 


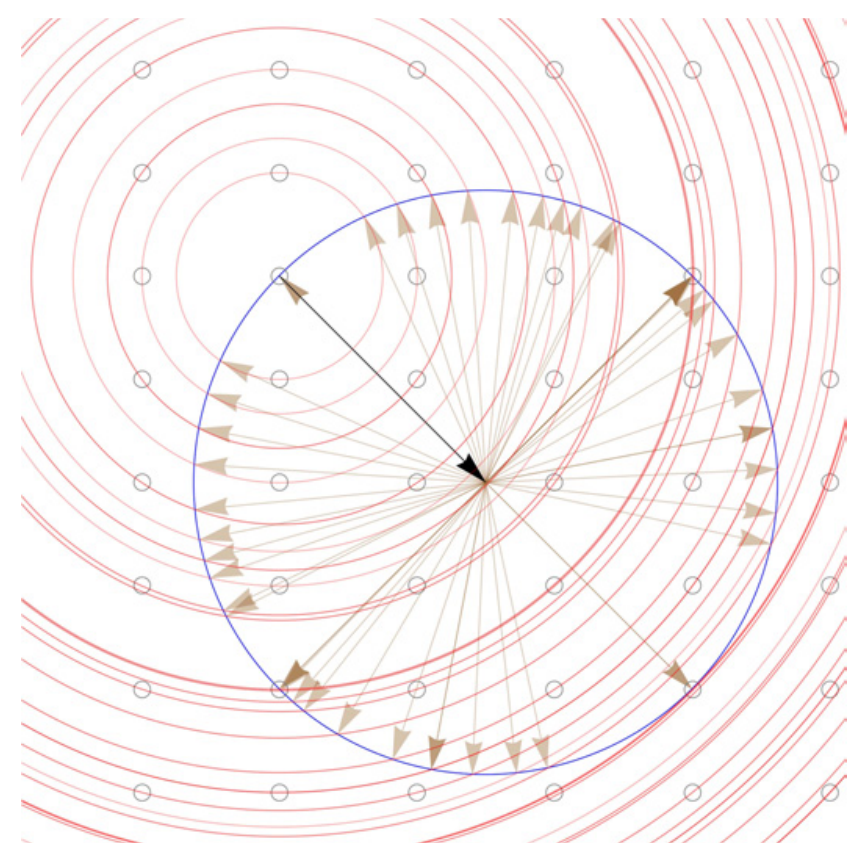

Figure 18. Ewald circle (blue) for the rotating-crystal method, with the same incident wave vector (black) in the same lattice plane as in Fig. 17. The rotation axis is perpendicular to the plane. The red circles are the orbits swept out by the reciprocal lattice vectors (grey circles). Each intersection with the Ewald circle represents a Bragg peak (brown arrows).

no sample rotation at all performed but the many rotation states are obtained by the presence of a very high number of randomly oriented small crystallites or grains, a polycrystalline sample or powder. The size of each single crystallite is still sufficient for diffraction to happen normally (but small crystallite sizes may eventually affect diffraction peak line shapes which can be exploited quantitatively to obtain microstructural information). With a fixed incident wave vector, the Ewald sphere stays fixed, and with the rotation (as it is physically not a rotation, one may rather use the term population) of the reciprocal lattice through all possible angles about the origin, each reciprocal lattice vector generates a sphere of the radius $K$ about the origin, as shown in Fig. 19. Each such a sphere will intersect the Ewald sphere in a circle, provided $K<2 k$. All vectors joining any point on the circle and the tip of the incident wave vector will be observed; they all lie on a so-called Debye-Scherrer cone. In most neutron powder diffraction experiments, where, a wide diffraction angle range is investigated, not the whole cones will be detected, but only a strip of a certain height in the so-called diffraction plane, which excels by favourable conditions in terms of resolution.

\subsection{Neutron diffractometers}

A generic diffractometer at a continuous neutron source works with a monochromatic incident neutron beam. The polychromatic, "white", beam, from a thermal neutron moderator (or a "hot" neutron moderator), arrives via a simple beam tube or a neutron guide at the monochromator. The monochromator is an array of equally aligned large single crystals with a mosaic fitting roughly the divergence of the incident polychromatic beam. In reflection or transmission position - the latter being disadvantageous but needing less material for low takeoff angles - the monochromator diffracts neutrons according to Bragg's law. Only monochromatic neutrons diffracted at a certain diffraction angle $2 \theta_{M}$, 


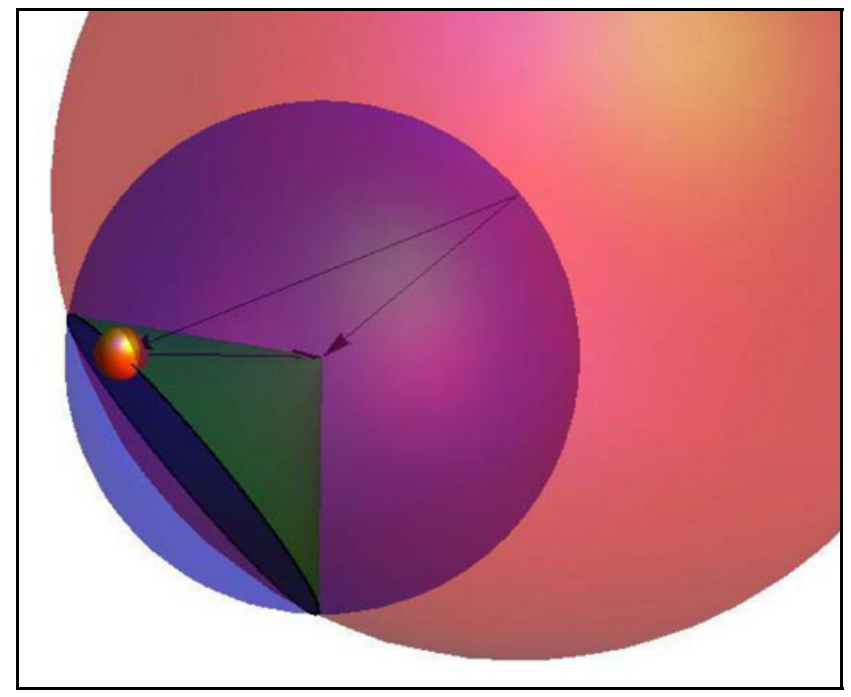

Figure 19. Ewald construction for the Debye-Scherrer method. The (here) smaller Ewald sphere (blue) intersects a sphere centred at the origin with a radius of $K$ for each lattice vector with $K<2 k$. Bragg peaks will appear for any wave vector connecting any point on the intersection circle with the tip of the incident wave vector. For each lattice vector the scattered rays therefore lie on a so-called Debye-Scherrer cone.

also called "takeoff angle", are allowed to proceed to the sample. After eventually transmitting a monitor, a detector of very low efficiency, whose counting rate is proportional to the incident flux, without being attenuated significantly, the monochromatic diffracted beam reaches the sample - a single crystal located at the center of an Euler cradle in the case of a four-circle single crystal diffractometer, or a usually cylindrical polycrystalline sample for a 2-axis "powder" diffractometer. This sample may well be placed inside some sample environment, a cryostat, furnace, pressure device or cryomagnet, to name a few.

According to Bragg's law, not only neutrons of one single wavelength $\lambda$ would be diffracted towards the sample, but also so-called higher harmonics $\lambda / n$, as the scattering vectors $\vec{k}$ and $n \vec{k}$ have the same direction. Although it may be that for some $n$ a Bragg reflection is forbidden for crystal symmetry reasons, but not for all. If now a wavelength $\lambda / n$, which is not forbidden, falls into the Maxwellian spectrum of the polychromatic thermal neutrons (plus the potential epithermal tail of undermoderated neutrons), a second wavelength will give rise to Bragg reflections from the sample, which cannot necessarily be distinguished from those of the main wavelength and lead potentially to wrong interpretations (such as superstructures). Luckily, it is possible to filter these contributions in many cases. As an example, a block of highly oriented pyrolytic graphite with the graphite planes perpendicular to the incident beam will stop most neutrons of wavelengths smaller than about $1.5 \AA$ by diffraction out of the incident beam direction. Such an optional filter can be placed in the polychromatic beam before the monochromator as well as in the monochromatic one afterwards.

\subsubsection{Single crystal diffractometers}

In the case of a four-circle diffractometer, the three angles of the Euler cradle are rotated to set the crystal to a specific orientation. A single detector, or a small flat or larger curved two-dimensional position-sensitive detector (PSD) records the diffracted beam within its angular range. The use of a PSD dramatically speeds up data collection. The sample is rotated step by step around the vertical axis ( $\omega$-scan) while the detector records the intensity profile of the diffracted beam. In the alternative 
$\omega-2 \theta$-scan the diffraction angle at which the detector is positioned (valid as such only for a single detector) is moved in a synchronized way. One can illustrate the different scanning modes with the Ewald construction.

When the intensities of a large enough number - typically 1000 to 100000 - of Bragg reflections have been collected, they can be used to compute the crystal structure of the sample, i.e., to determine the position and nature of its atoms.

For the solution of a crystal structure from this, one has to overcome, among others, the so-called phase problem: one measures the intensity, which is the square of the structure factor, and thus looses all phase information, i.e., the sign of the non-complex structure factor in the case of centrosymmetric structures or the complex nature (the distribution of real and imaginary part) of the structure factor in the other case. Here, we shall just mention the direct methods. This family of methods is supposed to estimate the (lost) phases of the Fourier transforms of the scattered intensity. One exploits statistical correlations between Bragg reflections: in certain triplets (and quadruplets) of reflections, the intensity and phase of two reflections explains the ones of the last one. For centrosymmetric structures, the product of the signs of two reflections of certain lattice vectors with the sign of a third one of the sum of the two lattice vectors, or the difference of the two lattice vectors is always positive (as long as the corresponding intensities are statistically meaningful - here comes in the statistical treatment). One can thus test a large number of different combinations of initial phases to find out the one or the few combinations resulting in self-consistent phases for all reflections. Obviously, the direct methods profit enormously with modern computing facilities. One alternative technique are the Patterson methods: The so-called Patterson function is a Fourier series with the observed intensities, thus, the squared structure factors, rather than the structure factors of unknown phase. This function, in the case of X-ray diffraction, shows a maximum at vectors where the same vector connects two maxima of electron density. It allows finding structural motifs formed by the heavier atoms (ideally, there are a few heavy atoms as compared to light atoms - for organic compounds one even tries to form salts of very heavy anions to provide "cornerstones" for a structure solution), and a consequent attribution of phases resulting from an incomplete structure solution. Iterative Fourier synthesis and evaluation of difference Fourier patterns allows the localization of missing atoms in the structural motif (this applies as well to the initial structure solution of direct methods).

For neutron diffraction, due to the existence of negative scattering lengths, the Patterson method does not necessarily work, and one can hardly avoid seeing all atoms at once, which makes its interpretation difficult. Similar applies for the direct methods; the potential existence of negative scattering lengths invalidates some of the exploited correlations. Also, the subsequent Fourier synthesis is hampered in the neutron case by a strong truncation effect: the constant scattering length leads to significant intensities up to the highest observed Bragg reflections, whereas the intensity of X-ray diffraction peaks approaches zero due to the atomic form factor, avoiding spurious artifacts, "truncation ripples", in the Fourier synthesis of electron density. In any case, neutron diffraction is rarely used for structure solution, not only for the aforementioned reasons (as there are other "brute force" methods today which would overcome them), but rather for the fact that one will typically consider scarcely available neutron beam time, once X-ray diffraction as a routine technique failed delivering the full picture. The preliminary result is even demanded when applying for beam time. It typically means that the structural skeleton is already known, but details, such as light atoms, and precision, in terms of thermal motion, atom positions and site occupancy, are missing and ought to be determined consecutively by neutron diffraction. In "X-n" studies, one uses systematically both probes to get a very precise picture of the bonded electron density distribution. Of course, neutron diffraction becomes close to unavoidable when it comes to magnetic structures. And here, the structure solution work will have to be done on neutron data alone - only that the atoms are already known, and with them the places of the magnetic moments, and only their orientation and size need to be determined. Together with symmetry constraints, once the so-called propagation vector is known, it boils down to the testing of a manageable number of allowed patterns 
of magnetic moment distributions. In some cases powder diffraction alone will not allow to distinguish several magnetic structures, for symmetry reasons they deliver precisely the same powder diffraction observation, and single crystal diffraction must be employed to make the difference (often then, the observation of a very limited number of Bragg reflections will be sufficient).

In general, a single crystal study provides a far more reliable and precise structural result as compared to the study of a polycrystalline product, a "powder". However, the later is normally more easily obtained, the diffraction experiment performed faster and the result sufficient to answer the questions having led to a structural study. In some cases even a powder diffraction study may provide a better result, e.g., when single crystals suffer twinning issues, i.e., the intergrowth of a second single crystal at a certain crystallographic orientation, or when a structural evolution during a phase transition or a chemical reaction needs to be followed in real time. In the later case, a single crystal normally does not even survive the transition, at least not without changing orientation, and even if it should, a realtime observation of a sufficiently large set of Bragg reflections in a simultaneous way is not possible, except in the case of Laue diffraction, which is an alternative technique to "traditional" four-circle diffractometers, currently enjoying a certain revival at neutron facilities.

\subsubsection{Powder diffractometers}

A constant wavelength neutron powder diffractometer on a continuous source follows the DebyeScherrer conception. Source, monochromator and sample (as well as the detector) define the diffraction plane. The monochromatic incident beam arrives at the polycrystalline sample, ideally a cylinder perpendicular to the plane, a few centimetres high $(2$ to $5 \mathrm{~cm})$ and a few millimetres in diameter (5 to $15 \mathrm{~mm}$ ). A powder would sit - ideally - inside a hollow cylinder of thin vanadium foil (very low coherent scattering cross section, therefore not adding Bragg peaks of considerable intensity to the powder diffraction pattern). In the case of strongly absorbing samples, one may use double-walled containers with the sample being placed in the small annular space between inner and outer cylinder of vanadium foil. In this case, the transmission becomes less dependent on the diffraction angle; the consequent absorption corrections are less sensitive to systematic errors.

\section{Data analysis}

There are many ways to analyse diffraction data. Structure solution from single crystal data has been briefly touched before; there is the refinement of structural data from the same data as well to be mentioned. These single crystal data treatments exist since long and are under constant evolution, e.g., in the field of proteins; there is a lot of software available, and frequently for free for the academic user.

The quantitative treatment of powder diffraction data is in some sense somewhat younger; here we shall mention the Rietveld method [20] and the "pdf-analysis", where "pdf' stands for pair distribution function, a recently developed method of treating powder data by the techniques, i.e., Fourier-transformations, established since long for the treatment of diffraction data of amorphous materials, e.g., glasses, and liquids, in order to obtain on top of the "classical" crystallographic structure data information on local disorder, also for "badly crystallised" and/or nanocrystalline materials. This later method exploits notably information about local order not visible in the long-range order, contained in the diffuse scattering outside or beneath the Bragg peaks [21]. Here again, for either method, one will find several software solutions, mostly free for academic use. One should mention here, that the aforementioned powder methods are originally structural refinement methods, but that it has become routine for about twenty years now also to solve structures alone form powder diffraction data, despite the lack of orientation information and the overlap of Bragg peaks.

The field is large and beyond the scope of this chapter. We shall therefore elucidate just one method, the Rietveld method, which became a real workhorse data analysis technique in the last few decades, 
accessible intellectually and in terms of software availability to a broad user community of experts and non-experts. It is a method that surely contributes a lot to an exhaustive analysis of diffraction experiments on polycrystalline materials relevant for energy conversion and transportation.

\subsection{Pattern matching}

With known wavelength and a known unit cell metrics of the sample, all possible angular positions of Bragg reflections in a given angular range of detection can be easily calculated and represented as deltafunctions (diffraction lines) in a calculated (powder) "diffraction pattern" (intensity versus diffraction angle).

Now, the real diffraction peak will not appear as a simple line, as a peak of infinitesimally narrow width. As we have stated before, the polychromatic neutron beam comes from a source of a finite size, meets a monochromator of a certain size and a certain mosaic. The monochromatic incident beam will meet a sample of finite size; detector cells of a certain width will detect the diffracted beam. Each time we deal with a certain divergence, which may be altered on each of these three paths by Soller collimators or similar means of divergence control; each time the beam is shaped differently. Mathematically, we deal with this through a convolution $\left(f(x) * g(x)=\int f(u) g(x-u) d u\right)$ of several shape components (e.g., triangular in the case of Soller collimators and trapezoidal in the case of simple slit collimation). The convolution of a sufficient number of shaping contributions eventually leads to a resulting shape, which can be described by a Gaussian peak shape. This is largely true for neutron powder diffraction and was a key to the success of the Rietveld method [22], which was largely developed on neutron powder diffraction data, where the Gaussian peak shape allowed a relatively easy computational treatment, whereas X-ray diffraction data have a more complex peak shape which was not easily treatable with the computational means of the 60 s and 70s. Thus, a convolution of a Gaussian peak profile with the diffraction pattern of delta-functions provides a more realistic computed pattern. However, we have to consider the different peak width at different diffraction angles. The angular dependence of the peak width $H_{G}$ (expressed as FWHM, the full width at half maximum) can be expressed after Caglioti [23] by three parameters $U, V$ and $W$.

$$
H_{G}^{2}=U \tan ^{2} \theta+V \tan \theta+W
$$

These parameters can be estimated from the relevant three divergences of a Debye-Scherrer powder diffractometer; one may note here, that the optimum of the peak width comes close to a diffraction angle identical to the take-off angle of the monochromator. The influence of the vertical divergence, which is considerable due to the frequent vertical focussing (to gain intensity) of neutron monochromators, is neglected here; in practice, it only becomes significant at rather low diffraction angles.

$$
\begin{gathered}
U=\frac{\alpha_{1}^{2}+\alpha_{2}^{2}}{\tan ^{2} \theta_{M}} \\
V=\frac{2 \alpha_{2}^{2}}{\tan \theta_{M}} \\
W=\alpha_{2}^{2}+\alpha_{3}^{2}
\end{gathered}
$$

Last not least, different Bragg peaks have different intensities. Before we try, in the next section, to compute these intensities, we could simply empirically vary them in order to produce a pattern close to an experimentally observed one. In practise, one can use the method of an iterative least squares minimization (because the system is not linear and parameters may correlate, which would be a condition for a least squares procedure to succeed in one go), where all the intensities of all Bragg 
reflections (overlapping peaks are simply sharing the intensity to same amounts) are refined in order to deliver the best fit to the observed pattern. Of course, in the same process, the parameters describing the peak width and either the wavelength or the unit cell metrics can be refined as well. To be (nearly) in perfect shape for a generic powder patter matching, one has to add a few other ingredients into the modelling, in particular a description of the background, frequently a simple polynomial expression with a constant term mainly proportional to the incoherent scattering contribution. By this so-called "Le Bail" approach [24] one obtains intensities for all observed Bragg reflections, analogue to what one would obtain from a single crystal experiment with the important exception of the arbitrary intensity repartition of overlapping peaks.

\subsection{Rietveld method}

The Rietveld method [20], properly spoken, has in mind more than a "whole-pattern-fitting" or "profile refinement", but the refinement of crystal structures. H. Rietveld [22] first worked out computer-based methods to make use of the full information content of powder patterns and, key to its success, shared the computer program, which today exists in various declinations, e.g. FullProf [25].

The diffraction pattern is recorded in digitized form, i.e., as numerical intensity values $y_{\mathrm{i}}$ at each of several hundred or thousand increments $i$ (originally corresponding to equidistant steps of an angular single-detector scan, today frequently to not necessarily equidistant physical or virtual cells of a PSD) in the pattern. These increments may be either in some energy parameter, such as velocity for time-offlight neutron data, or, in the here discussed constant wavelength method, in scattering angle $2 \theta$. Typical step sizes range form $0.01^{\circ}$ to $0.05^{\circ}$ for fixed-wavelength $\mathrm{X}$-ray data and $0.025^{\circ}$ to $0.2^{\circ}$ for neutron data.

\subsubsection{The least squares minimization}

The quantity minimized in the least squares refinement is the residual $\chi^{2}$ :

$$
\chi^{2}=\sum_{i=1}^{n} w_{i}\left[y_{i}-y_{c i}(\vec{\beta})\right]^{2}
$$

Here, we have computed intensities $y_{c i}$ from a vector $\vec{\beta}$ of crystallographic and instrumental parameters, as well as a weighting scheme, normally inversely proportional to the square of the standard deviation of each observation, $w_{i}=1 / \sigma_{i}^{2}$, which is close to $w_{i}=1 / y_{i}$ for large counting rates, as long as the count rage obeys the Poisson distribution.

The least squares minimization procedure consists in finding the minimum of the residual, thus, fulfilling the condition $\frac{\partial \chi^{2}}{\partial \vec{\beta}}$. Through a Taylor expansion of $y_{c i}(\vec{\beta})$ around $\vec{\beta}$ it leads to a set of normal equations involving derivatives of all the calculated intensities with respect to each refinable parameter $\beta_{j} \in \vec{\beta}$.

$$
\boldsymbol{A} \cdot \Delta \vec{\beta}=\vec{b} \quad \text { with } \quad A_{j k}=\sum_{i=1}^{N} w_{i} \frac{\partial y_{c i}(\vec{\beta})}{\partial \beta_{j}} \frac{\partial y_{c i}(\vec{\beta})}{\partial \beta_{k}} \quad \text { and } \quad \sum_{i=1}^{N} 2 w_{i}\left[\left(y_{i}-y_{c i}\right) \frac{\partial^{2} y_{c i}(\vec{\beta})}{\partial \beta_{j} \partial \beta_{k}}\right]
$$

One obtains the solution by inversion of the normal matrix with elements $M_{j k}$ given by the following expression:

$$
M_{j k}=-\sum_{i=1}^{N} 2 w_{i}\left[\left(y_{i}-y_{c i}\right) \frac{\partial^{2} y_{c i}(\vec{\beta})}{\partial \beta_{j} \partial \beta_{k}}-\frac{\partial y_{c i}(\vec{\beta})}{\partial \beta_{j}} \frac{\partial y_{c i}(\vec{\beta})}{\partial \beta_{k}}\right]
$$


The whole story is the creation and inversion of a $P \times P$ matrix with $P$ being the number of parameters being refined, i.e., the number of vector elements in $\vec{\beta}$. As the residual function is not linear, the solution must be found in an iterative procedure with parameters shifting each time by $\Delta \beta_{k}$ computed as follows through solving the normal equations:

$$
\Delta \beta_{k}=\sum_{j=1}^{P} M_{j k}^{-1} \frac{\partial \chi^{2}}{\partial \beta_{k}}
$$

The shifts of the parameters are added to the starting ones to give rise to a new set, considered as starting one for a next cycle. Frequently, a damping factor $0<\alpha<1$ is applied to the shift application to avoid divergence: $\vec{\beta}^{\prime}=\vec{\beta}+\alpha \cdot \Delta \vec{\beta}$. The process is repeated until a convergence criterion is fulfilled. The variance $\sigma^{2}$ (squared estimated standard estimation) of the adjusted parameters is obtained as follows:

$$
\sigma^{2}\left(\beta_{k}\right)=\left(\boldsymbol{A}^{-1}\right)_{k k} \cdot \chi_{v}^{2} \quad \text { with } \quad \chi_{v}^{2}=\frac{\chi^{2}}{N-P}
$$

\subsubsection{The profile of powder diffraction patterns}

Typically, several Bragg reflections (not only overlapping, but also neighbouring ones) contribute to an intensity $y_{i}$. The computed intensities $y_{c i}$ are determined from the structure factors as resulting from the structural model:

$$
y_{c i}=s \sum_{\vec{h}} I_{\vec{h}} \cdot \phi_{i, \vec{h}}\left(2 \theta_{i}-2 \theta_{\vec{h}}\right)+y_{b i}
$$

In this formulation, we have a refinable scale factor $s$, the intensity of each Bragg peak $I_{\vec{h}}\left(\overrightarrow{\beta_{I}}\right)$, a background contribution $y_{b i}\left(\overrightarrow{\beta_{B}}\right)$ at each step (composed from incoherent and diffuse scattering of the sample itself, further scattering from air and the sample environment and the detector's noise, with a subset of refinable parameters $\overrightarrow{\beta_{B}}$, frequently parameters of an empirical polynomial expression), and a normalised profile function $\phi\left(\overrightarrow{\beta_{p}}\right)$ with $\int_{-\infty}^{\infty} \phi(x) d x=1$, characterized by its width, i.e., its full width at half maximum, and other shape parameters. The index at the profile function $\phi$ shall indicate, that its width (and potentially other parameters) depends on the angular position $2 \theta_{\mathrm{i}}$ through the instrumental resolution function (or more general shape function) and on the lattice vector $\vec{h}$ in the case of isotropic or anisotropic peak broadening due to microstructural details like particle size and shape distribution and microstrain. Refinable instrumental and micro-structural parameters are contained in a subset $\overrightarrow{\beta_{P}}$ of refinable profile parameters. Finally, $2 \theta_{\vec{h}}\left(\overrightarrow{\beta_{M}}\right)$, contains the information about the crystal's unit cell through Bragg's equation and several sources of a diffraction angle offset, such as a sample displacement or the detector's zero-shift.

\subsubsection{The integrated intensity containing the structural information}

The intensity $I_{\vec{h}}\left(\overrightarrow{\beta_{I}}\right)$ contains structural information (subset $\overrightarrow{\beta_{I}}$ of the vector $\vec{\beta}$ of refinable parameters) on atom positions, magnetic moments, site occupancies and atomic displacement through the contributing structure factor $F_{\vec{h}}\left(\vec{\beta}_{I}\right)$, further the Lorentz, polarisation and multiplicity factors $L_{\vec{h}}$, the preferred orientation function $P_{\vec{h}}$ and an absorption factor, typically dependent on the scattering angle:

$$
I_{\vec{h}}=L_{\vec{h}} \cdot\left|F_{\vec{h}}\right|^{2} \cdot P_{\vec{h}} \cdot A_{\vec{h}}
$$


In the case of neutron diffraction, the Lorentz-polarisation factor ${ }^{28}$ is $1 / 2 \sin ^{-2} \theta \cos ^{-1} \theta$, as the polarisation ratio $K$ is zero for neutrons in the following expression:

$$
L_{\vec{h}}=\frac{1-K+K \cos ^{2} 2 \theta_{M} \cos ^{2} 2 \theta}{2 \sin ^{2} \theta \cos \theta}
$$

The preferred orientation (or texture) plays a minor role in neutron powder diffraction, as, due to the large sample size and the Debye-Scherrer scattering geometry, one manages easily to avoid it. Nevertheless, strongly anisotropic particles (platelets, needles) may lead to some preferred orientation, as well as, e.g., the application of magnetic or electric field, even for neutron diffraction. The simple, empirical March-Dollase function expresses a correction as a function of two parameters, an angle $\alpha$ between the lattice vector and the orientation axis and an orientation ratio $r$ with $r<1$ for platelets and $r>1$ for needles:

$$
P_{\vec{h}}=\left(r^{2} \cos ^{2} \alpha+r^{-1} \sin ^{2} \alpha\right)^{-3 / 2}
$$

The absorption correction $A$ corresponds to the transmission at a given diffraction angle. For a cylindrical powder sample of radius $R$ with a linear absorption coefficient $\mu$ (concentration times cross section) we have:

$$
A_{\vec{h}} \approx e^{-\left(1.7133-0.0368 \sin ^{2} \theta_{\vec{h}}\right) \mu R+\left(0.0927+0.375 \sin ^{2} \theta_{\vec{h}}\right)(\mu R)^{2}}
$$

The structure factor, finally, contains the refinable structural parameters: atom positions $\vec{r}_{j}$ (up to 3 fractional coordinates for $n$ sites), occupation factors $O_{\mathrm{j}}$ for up to $n-1$ sites and up to $n$ isotropic (in the simplest case) displacement factors $B_{\mathrm{j}}$ :

$$
F_{\vec{h}}=\sum_{j=1}^{n} O_{j} \bar{b}_{j} T_{j}\left(\theta_{\vec{h}}\right) e^{2 \pi i \cdot \vec{h} \cdot \vec{r}_{j}} \quad \text { with } \quad T_{j}(\theta)=e^{-B_{j} \frac{\sin ^{2} \theta}{\lambda^{2}}} .
$$

\subsubsection{Microstructure analysis}

The peak shape function $\phi_{i, \vec{h}}\left(2 \theta_{\mathrm{i}}-2 \theta_{\vec{h}}\right)$ contains the profile parameters, and through $2 \theta_{\vec{h}}$ the unit cell parameters, determining the peak positions. In most cases of neutron powder diffraction, $\phi$ is well approached by a convolution of (intrinsic) Lorentzian $L$ and (instrumental) Gaussian $G$ profiles: $V(x)=$ $G(x) * L(x)=\int_{-\infty}^{\infty} L(x-u) G(u) d u$. This so-called Voigt-function $V$ is sufficiently approached by the pseudo-Voigt function $p V$, a linear combination of both contributors, which has the advantage of being less computing-intense: $p V(x)=\eta L(x)+(1-\eta) G(x)$. Whereas $G$ and $L$ depend on their width (expressed as full width at half maximum, FWHM, or as integral breadth $\beta=\frac{\int_{\infty}^{\infty} \phi(x) d x}{\phi(0)}>F W H M$, the width of a rectangle with same height as the peak and same area), $G\left(x, H_{G}\right)$ and $L\left(x, H_{L}\right)$, the Voigt function depends on both widths, $V\left(x, H_{G}, H_{L}\right)$, and the pseudo-Voigt function on its width and mixing factor $\eta, p V(x, H, \eta)$. If one wants correct in an individual peak fitting approach a peak width for its instrumental contribution, one has to keep in mind that in a convolution, Lorentzian widths (or breadths) can be summed up, whereas for Gaussian widths, one has to sum up the squared width to obtain the squared width of the convolution. One can exploit simple $2 \theta$-dependencies of integral breadth

\footnotetext{
${ }^{28}$ Frequently, the so-called multiplicity is integrated together with the Lorentz-polarization factor, although it has a very different origin. The multiplicity groups symmetry equivalent lattice vectors together. For cubic symmetry, the factor may go up to 48 for reflections with three different Miller indices. Obviously, it is not necessary to deal with symmetry-equivalent reflections this way, but it means a gain in computing efficiency of about an order of magnitude, which is not negligible in a refinement algorithm, where structure factors containing transcendental functions have to be computed many thousands of times.
} 
to separate peak broadening due to particle size and strain: $\beta_{\text {size }}=\frac{\lambda}{D \cos \theta}$ (the so-called Scherrer formula with the apparent crystallites size $D$ ) and $\beta_{\text {strain }}=4 e \tan \theta$ with the so-called maximum strain $e=\Delta d / d$, which is by $25 \%$ bigger than the root mean square strain [26]. One can go much further in peak profile analysis in the frame of the Rietveld refinement and refine models of anisotropic strain broadening [27] or use spherical harmonics to model the anisotropic shape of crystallites.

\section{Concluding remark}

Now, I can only encourage to apply the powerful method of (neutron) diffraction to a real case: take your powdered material, if you can spare a gram of it, bring it to a neutron diffraction instrument (if it is more than just a quick test, you will have to write a beam time demand proposal, otherwise you just contact the scientific staff at one of the sources) or just to the X-ray powder diffractometer in some research institute, record a pattern, download a Rietveld refinement software such as FullProf [25] from the Internet, follow the provided tutorials or take one of the provided examples and apply it to your investigated case, and try to refine some parameters. Ideally, you'll see how nicely this works to get some information out of your powder. Now, let your imagination play: how does your powder behave when exposed to oxygen? What happens, when you heat it up? How does it behave at high pressure? How fast does it absorb hydrogen at different temperatures? What is the particle size distribution of the powder? Does the powder contain several phases? Provided, we have an oxide, does it have any interstitial oxygen? Are there any vacancies on the oxygen positions? All these questions can probably be answered easily by follow-up neutron diffraction experiments; the main bottleneck may be only your imagination!

I thank the organisers of the neutron school on "neutrons et matériaux pour l'énergie" in Sète in 2013, preceding the "Journées de Diffusion Neutronique", JDN21, in particular Monica Ceretti, Werner Paulus and Clemens Ritter for the kind invitation to give a lecture on this fundamental chapter, and in consequence to have given me the opportunity to write this chapter up. Of course, it has nothing innovative, and the interested reader will find the information in many places, in textbooks, lecture scripts and on the Internet, but it is such an important chapter for any scientist, that it cannot be repeated enough and this is just another approach, the one of an inorganic solid state chemist, needing diffraction methods as a unique tool to understand the structure of metal. I thank further my colleagues Juan Rodriguez-Carvajal, Werner F. Kuhs, Helmut Schober and Tapan Chatterji for inspiration through lectures and discussions.

\section{References}

[1] G. Binnig, H. Rohrer, Scanning tunneling microscopy, 126 (1983) 236-244. doi:10.1016/00396028(83)90716-1.

[2] G. Binnig, C.F. Quate, C. Gerber, Atomic force microscope (1986). doi:10.1103/ PhysRevLett.56.930.

[3] P.W. Atkins, R. Friedman, Molecular Quantum Mechanics, 5 ed., Oxford University Press, 2011.

[4] D. Halliday, R. Resnick, K.S. Krane, Physics, Volume 2, 5 ed., Wiley, 2002.

[5] H. Schober, S. Rols, Les excitations dans la matière condensée : vibrations et phonons, 10 (2010) 3-136. doi:10.1051/sfn/2010001.

[6] L. Koester, W.B. Yelon, Compilation of Low Energy Neutron Scattering Lengths and Cross Sections, ECN, 1982.

[7] S. Mughabghab, Neutron Cross Sections, 1 (2012) 828.

[8] V. McLane, Neutron Cross Sections, 2 (2012) 850.

[9] R.P. Feynman, Diffraction, in: Feynman Lectures on Physics, Volume 1, Addison Wesley Publishing Company, Reading, Massachusetts, 1963. 
[10] J.D. Patterson, B.C. Bailey, Solid-State Physics (2010) 849.

[11] M.J. Buerger, Elementary crystallography, Wiley, New York, 1956.

[12] N.W. Ashcroft, D. Mermin, Solid State Physics, 1st ed., Thomson Learning, Stamford, 1976.

[13] A.R. West, Solid State Chemistry and its Applications, John Wiley \& Sons, 2014.

[14] G.L. Squires, Introduction to the Theory of Thermal Neutron Scattering, Cambridge University Press, 2012.

[15] W. Marshall, S.W. Lovesey, Theory of thermal neutron scattering: the use of neutrons for the investigation of condensed matter (1971) 599.

[16] L. Van Hove, Correlations in Space and Time and Born Approximation Scattering in Systems of Interacting Particles, Phys Rev. 95 (1954) 249-262. doi:10.1103/PhysRev.95.249.

[17] G. Placzek, The Scattering of Neutrons by Systems of Heavy Nuclei, Phys Rev. 86 (1952) 377-388. doi:10.1103/PhysRev.86.377.

[18] J. Yarnell, M. Katz, R. Wenzel, S. Koenig, Structure Factor and Radial Distribution Function for Liquid Argon at $85^{\circ}$ K, Phys. Rev. A. 7 (1973) 2130-2144. doi:10.1103/PhysRevA.7.2130.

[19] P.P. Ewald, Introduction to the dynamical theory of X-ray diffraction, Acta Cryst A. 25 (1969) 103-108. doi:10.1107/S0567739469000155.

[20] R.A. Young, R.A. Young, The Rietveld Method, Oxford University Press, 1995.

[21] T. Egami, S.J.L. Billinge, Underneath the Bragg Peaks, Newnes, 2012.

[22] H.M. Rietveld, A profile refinement method for nuclear and magnetic structures, J Appl Cryst. 2 (1969) 65-71. doi:10.1107/S0021889869006558.

[23] G. Caglioti, A. Paoletti, F.P. Ricci, Choice of collimators for a crystal spectrometer for neutron diffraction, Nuclear Instruments. 3 (1958) 223-228. doi:10.1016/0369-643X(58)90029-X.

[24] A. Le Bail, Whole powder pattern decomposition methods and applications: A retrospection, Powder Diffraction. 20 (2005) 316. doi:10.1154/1.2135315.

[25] J. Rodríguez-Carvajal, Recent Developments of the Program FULLPROF, Com Pow Diff (IUCr) Newslett. 26 (2001) 12-19.

[26] E.J. Mittemeijer, P. Scardi, Diffraction Analysis of the Microstructure of Materials, Springer Science \& Business Media, 2004.

[27] J. Rodríguez-Carvajal, M.T. Fernandez-Diaz, J.L. Martínez, Neutron diffraction study on structural and magnetic properties of La2NiO4, J Phys Condens Matter. 3 (1991) 3215-3234. doi:10.1088/0953-8984/3/19/002. 